Aus der Klinik für Klinische Neurophysiologie

(Prof. Dr. med. W. Paulus)

im Zentrum Neurologische Medizin

der Medizinischen Fakultät der Universität Göttingen

\title{
Exzitabilitätsuntersuchungen von Motoneuronen
}

an Patienten mit

benignem Faszikulations-Crampus-Syndrom

und die Funktion von $\mathrm{HCN}-$ Kanälen

\author{
INAUGURAL - DISSERTATION \\ zur Erlangung des Doktorgrades \\ der Medizinischen Fakultät der \\ Georg-August-Universität zu Göttingen
}

vorgelegt von

Melanie Bathon, geb. Wagenknecht

aus Kassel

Göttingen 2016 
Dekan:

Prof. Dr. rer. nat. H. K. Kroemer

I. Berichterstatter: Prof. Dr. Walter Paulus

II. Berichterstatter/in: Prof. Dr. Swen Hülsmann

III. Berichterstatter/in: Prof. Dr. Rainer Mausberg

Tag der mündlichen Prüfung: 15.02.2016 


\section{Inhaltsverzeichnis}

1 Einleitung...........................................................................................................

1.1 Benignes Faszikulations-Crampus-Syndrom .................................................

1.1.1 Diagnostik des benignen Faszikulations-Crampus-Syndroms.......................2

1.2 Pathophysiologie von Faszikulationen und Krämpfen.....................................4

1.2.1 Haben Muskelkrämpfe einen zentralen oder peripheren Ursprung?..............4

1.2.2 Autoimmunologische Theorien........................................................

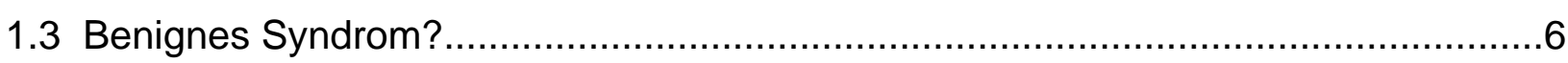

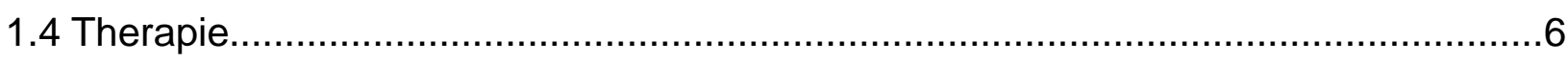

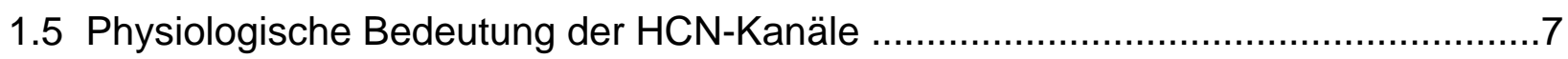

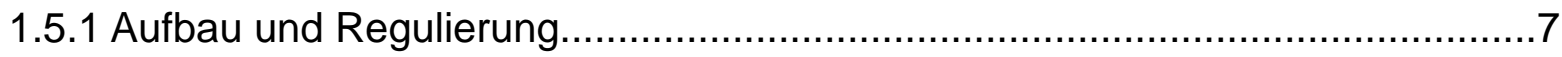

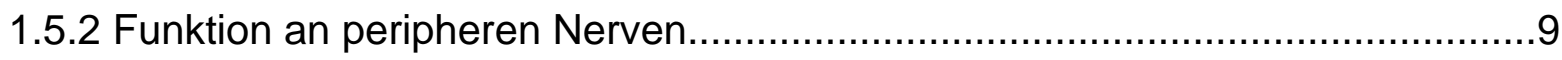

1.5.3 Funktion am Herzen................................................................... 10

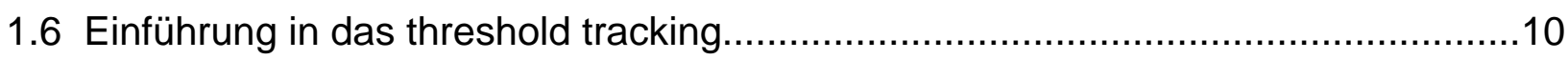

1.6.1 Entwicklung des threshold trackings.................................................. 10

1.6.2 Threshold tracking im Vergleich zur neurophysiologischen

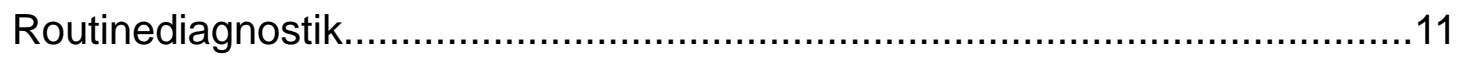

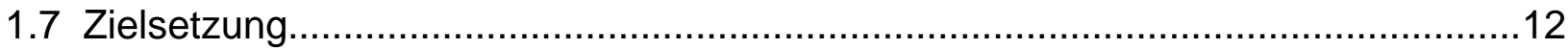

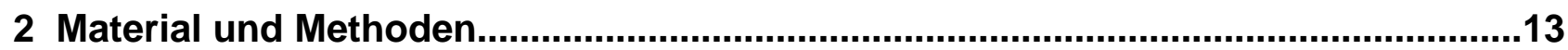

2.1 Probandenkollektiv und Studiendesign..................................................... 13

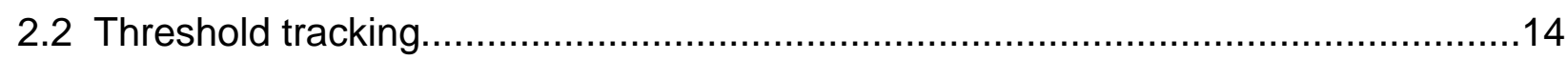

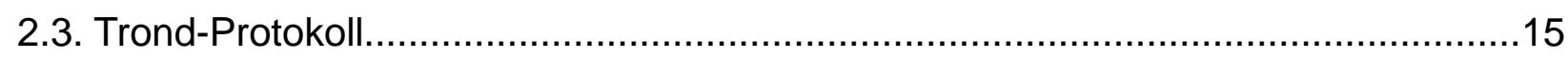

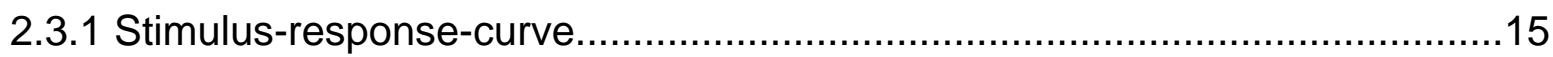

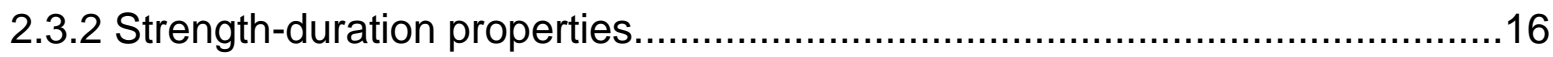

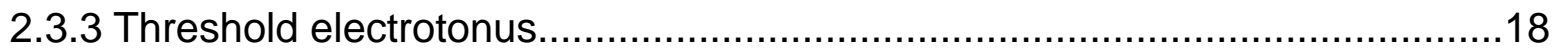

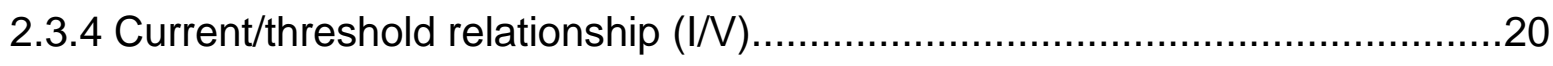

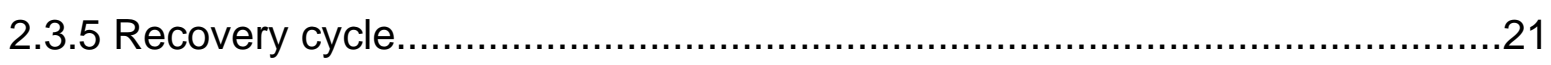

2.3 Definitionen der Exzitabilitätsparamter....................................................23

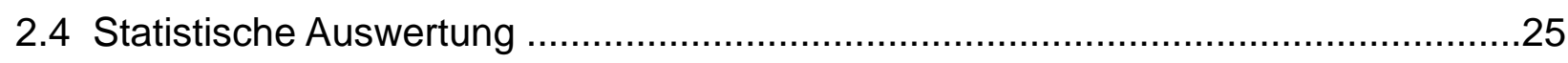




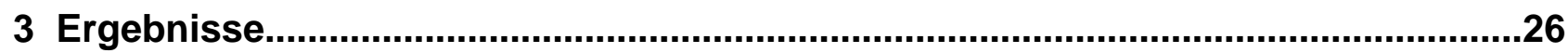

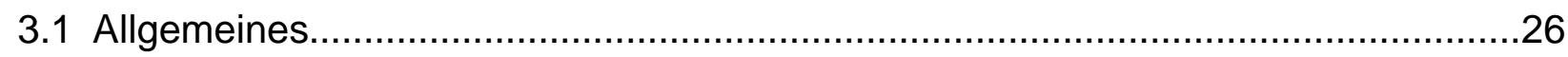

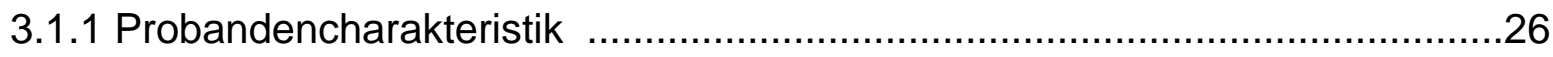

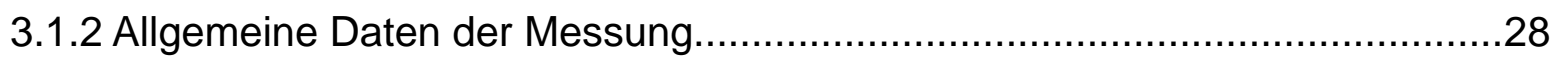

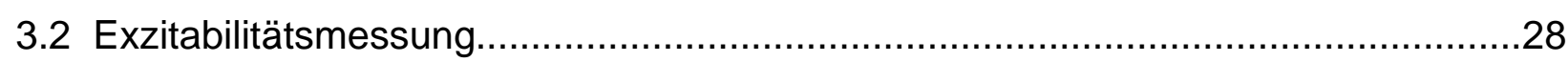

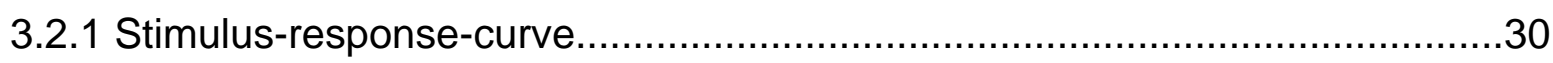

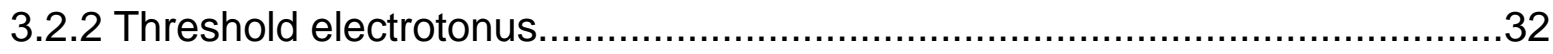

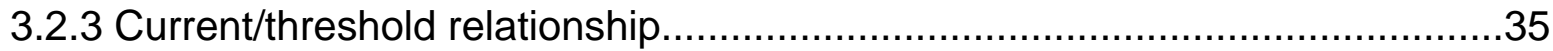

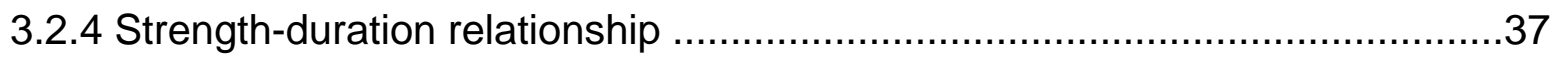

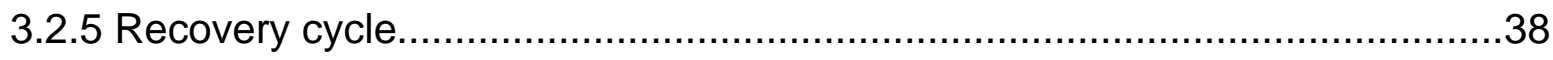

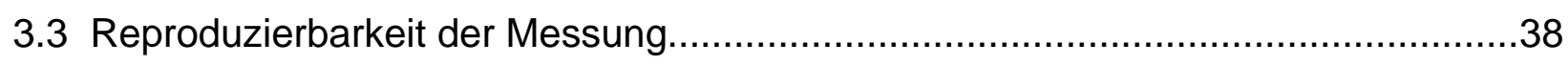

3.4 Die wichtigsten Ergebnisse im Überblick......................................................40

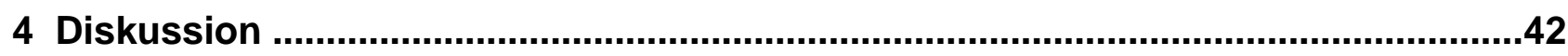

4.1 Veränderungen der axonalen Erregbarkeit bei Patienten mit einem benignen

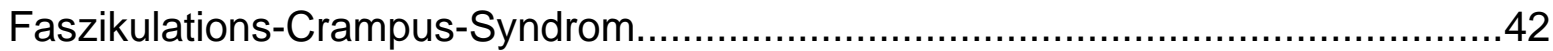

4.2 Ergebnisse im Zusammenhang bisheriger Studien zum benignen FaszikulationsCrampus-Syndrom

4.3 Vergleich der Ergebnisse zu anderen Erkrankungen mit Faszikulationen und

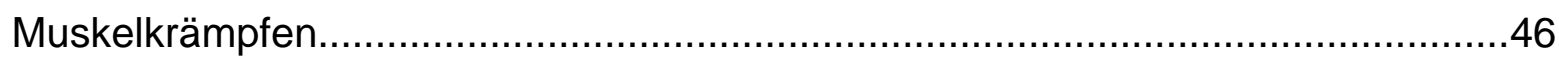

4.3.1 Amyotrophe Lateralsklerose (ALS) ....................................................

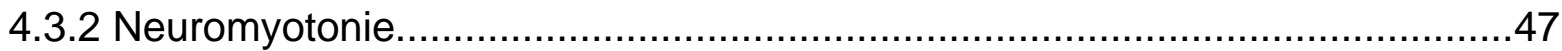

4.4 Ist die veränderte inward rectification die Ursache oder das Ergebnis einer peripheren Übererregbarkeit?

4.4.1 Bedeutung der inward rectification sensibler Neurone im Vergleich zu den Ergebnissen dieser Arbeit...................................................................... 48

4.4.2 Vergleich der Ergebnisse mit low-threshold Motoraxonen............................50

4.4.3 Pathophysiologische Rolle der axonalen HCN-Kanäle.................................50

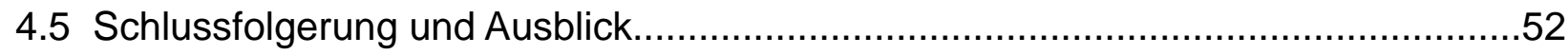

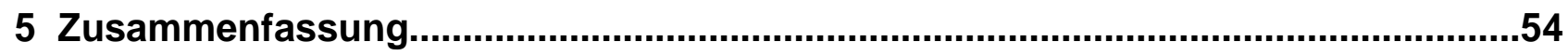

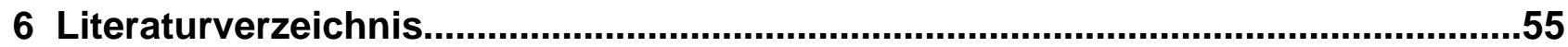




\section{Abkürzungsverzeichnis}

Abb.

AIP

ALS

Abbildung

BFCS

bzw.

akut intermittierende Porphyrie

${ }^{\circ} \mathrm{C}$

amyotrophe Lateralsklerose

$\mathrm{Ca}^{2+}$

benignes Faszikulations-Crampus-Syndrom

beziehungsweise

cAMP

Grad Celsius

$\mathrm{cm}$

Kalzium

CNBD

cyclic adenosine monophosphate

$\mathrm{Cs}^{+}$

Zentimeter

d

df

cyclic nucleotide-binding domain

DML

Caesium

depolarisierend

degrees of freedom

EKG

distal motorische Latenz

EMG

Elektrokardiogramm

GTG

$f$

$\mathrm{h}$

Elektromyogramm

Glycin-Tyrosin-Glycin

funny

hyperpolarisierend

$\mathrm{HCN}$

hyperpolarization-activated cyclic nucleotide-gated cation channel

$\mathrm{Hz}$

Hertz

I

Strom

$\mathrm{K}^{+}$

Kalium

k.A.

keine Angaben

M.

$\mathrm{mA}$

MAP

Musculus

Milliampere

mg

mitogen-activated-protein

$\min$

Milligramm

ms

Minute

Millisekunde

$\mathrm{mV}$

Millivolt

MW

Mittelwert

N.

Nervus 


$\begin{array}{ll}\mathrm{Na}^{+} & \text {Natrium } \\ \mathrm{NC} & \text { normal control } \\ \mathrm{NLG} & \text { Nervenleitgeschwindigkeit } \\ \mathrm{OE} & \text { obere Extremität } \\ \mathrm{PIP}_{2} & \text { Phosphatidylinositol-4,5-bisphosphat } \\ \mathrm{RRP} & \text { relatively refractory period } \\ \mathrm{S.} & \text { siehe } \\ \mathrm{SE} & \text { standard error } \\ \mathrm{TE} & \text { threshold electrotonus } \\ \mathrm{UE} & \text { untere Extremität } \\ \mathrm{UK} & \text { United Kingdom } \\ \text { V } & \text { Spannung } \\ \text { VGKC } & \text { voltage-gated potassium channels } \\ \text { vgl. } & \text { vergleiche }\end{array}$




\section{Abbildungsverzeichnis}

Abb. 1 Aufbau des HCN-Kanals 8

Abb. 2 Anordnung der Elektroden zur Messung des rechten N. medianus 15

Abb. 3 Bedeutung von Rheobase und Chronaxie 17

Abb. 4 Bedeutung des strength-duration time constant 17

Abb. 5 Phasen des Threshold electrotonus 19

Abb. 6 Bedeutung I/V-Kurve 21

Abb. 7 Phasen des Recovery cycles 22

Abb. 8 Beispiel für die Messung eines Probanden 23

Abb. 9 Stimulus-response-curve 31

Abb. 10 (A) Kompletter Threshold electrotonus 33

(B) $+40 \%$ depolarisierender Reiz des TE

Abb. 11 (A) -20\% hyperpolarisierender Reiz desTE

(B) $-40 \%$ hyperpolarisierender Reiz desTE

Abb. 12 (A) -70\% hyperpolarisierender Reiz desTE

(B) $-100 \%$ hyperpolarisierender Reiz desTE

Abb. 13 (A) und (B) Current/threshold relationship 36

Abb. 14 (A) Strength-duration time constant 37

(B) Recovery cycle

Abb. 15 Reproduzierbarkeit der Messung 39

Abb. 16 Wichtigsten Ergebnisse im Überblick 41 


\section{Tabellenverzeichnis}

Tab. 1 Definitionen der Exzitabilitätsparameter 24

Tab. 2 Informationen zu den Beschwerden der einzelnen BFCS- 27 Patienten

Tab. 3 Vergleich der Exzitabilitätsparameter des N. medianus 29 zwischen Patienten mit einem BFCS und einer gesunden Kontrollgruppe 


\section{Einleitung}

\subsection{Benignes Faszikulations-Crampus-Syndrom}

Das benigne Faszikulations-Crampus-Syndrom (BFCS) ist gekennzeichnet durch häufige Muskelkrämpfe und Faszikulationen, die sich meist nach körperlicher Anstrengung intensivieren und vor allem nachts auftreten (Tahmoush et al. 1991, de Carvalho und Swash 2004). Die Diagnose kann gestellt werden, wenn zuvor alle anderen Ursachen für Faszikulationen und Muskelkrämpfe ausgeschlossen wurden.

Faszikulationen sind schmerzlose, spontane Entladungen von motorischen Einheiten, die sich durch sichtbare Muskelzuckungen bemerkbar machen, wenn sie in oberflächlichen Muskelschichten entstehen (Van der Heijden et al. 1994, Desai und Swash 1997). Durch Klopfen und Kneifen sind Faszikulationen bei einigen Personen provozierbar (Zierz 2003). Benigne Faszikulationen sind ein häufiges Phänomen. Es wird davon ausgegangen, dass um die $40 \%$ der gesunden Bevölkerung betroffen sind und es gerade bei älteren Personen gehäuft auftritt (Fermont et al. 2010).

Muskelkrämpfe sind definiert als plötzlich einsetzende schmerzvolle Muskelkontraktionen, die entweder spontan oder nach Willkürinnervation des betroffenen Muskels ausgelöst werden und von alleine oder durch Dehnung des kontrahierten Muskels nachlassen (Tahmoush et al. 1991, Zierz 2003). Sie halten meist für einige Sekunden bis mehrere Minuten an (Miller und Layzer 2005). Muskelkrämpfe scheinen, gerade bei Patienten über 65 Jahre, mit einer Prävalenz zwischen 35\% und 60\% noch häufiger als die benignen Faszikulationen aufzutreten (Naylor und Young 1994, Lopate et al. 2013). Die Ätiologie der Muskelkrämpfe variiert dabei sehr stark. So treten sie bei gesunden Personen auf, häufig belastungsabhängig aber auch idiopathisch und im Rahmen vieler Erkrankungen wie Neuropathien, Motoneuronerkrankungen, Radikulopathien, metabolischen Erkrankungen wie extrazellulärer Volumenmangel und Urämie, endokrinen Veränderungen, wie zum Beispiel im Rahmen einer hypo- oder hyperthyreoten Stoffwechsellage, bei Vorliegen von Antikörpern gegen spannungsabhängige Kalium $\left(\mathrm{K}^{+}\right)$-Kanäle (VGKC = voltage-gated potassium channels), familiären Muskelkrampferkrankungen, Schwangerschaft und peripherer Nervenübererregbarkeit wie der Neuromyotonie (Caress und Walker 2002, 
Miller und Layzer 2005, Lopate et al. 2013). Des Weiteren treten Muskelkrämpfe als Nebenwirkungen vieler Medikamente auf, wie zum Beispiel bei der Einnahme von Nifedipin, Salbutamol, Cimetidin und Bumetaniden (Keidar et al. 1982, Lane und Mastaglia 1978). Somit müssen, bevor die Diagnose eines BFCS gestellt werden kann, etliche Ursachen für Muskelkrämpfe ausgeschlossen werden. Obwohl Faszikulationen und Muskelkrämpfe als einzelne Symptome sehr häufig sind, scheint die Kombination aus häufigen Faszikulationen und häufigen Muskelkrämpfen, wie sie im Rahmen des BFCS auftreten, nur bei $0,4-0,7 \%$ der Bevölkerung aufzutreten (Jansen et al. 1991). Der Erkrankungsbeginn liegt im Mittel bei etwa 45 Jahren (Liewluck et al. 2014).

Klinisch zeigen BFCS-Patienten neben den Faszikulationen und Muskelkrämpfen weder eine Atrophie noch eine Schwäche der betroffenen Muskelgruppen, und auch die Sensibilität und die Muskeleigenreflexe sind normal (Tahmoush et al. 1991, de Carvalho und Swash 2004). Für die Patienten, die unter diesem Syndrom leiden, kann es eine erhebliche Minderung der Lebensqualität bedeuten, da die Muskelkrämpfe zum einen sehr schmerzhaft sind und zum anderen durch das häufige Auftreten in der Nacht der Schlaf massiv beeinträchtigt wird und es somit eher zu einer Tagesmüdigkeit kommen kann (Harrison und Benatar 2007). Des Weiteren besteht eine Assoziation der Krämpfe mit körperlicher Anstrengung, wodurch es zu Einschränkungen sowohl im beruflichen als auch im privaten Bereich kommen kann.

\subsubsection{Diagnostik des benignen Faszikulations-Crampus-Syndroms}

Die durchzuführende Diagnostik bei dem Verdacht auf ein BFCS beruht vor allem auf klinisch neurologischen und elektrophysiologischen Untersuchungen, die ein Elektromyogramm (EMG), die Messung der Nervenleitgeschwindigkeit (NLG) und der distal motorischen Latenz (DML) beinhalten, um andere Ursachen für die Faszikulationen und Muskelkrämpfe auszuschließen.

Im EMG mittels Nadelelektroden können sich Faszikulationen zeigen, die dann Faszikulationspotentiale genannt werden (Desai und Swash 1997). Dabei kommt es zur Kontraktion einer kompletten motorischen Einheit und nicht nur einzelner Muskelfasern (Vogel 2011). Die abgeleiteten Potentiale entsprechen demnach Potentialen motorischer Einheiten, welche allerdings spontan und ohne Willkürinnervation auftreten (Van der 
Heijden et al. 1994, Vogel 2011). Des Weiteren können sich auch Muskelkrämpfe im EMG zeigen in Form von wiederholten hochfrequenten Entladungen motorischer Einheiten, wobei sich sowohl zu Beginn als auch am Ende des Krampfes Faszikulationspotentiale zeigen können (Lopate et al. 2013).

Die Messung der NLG und der DML sollte bei diesen Patienten keinerlei Auffälligkeiten zeigen, ebenso wie die klinisch neurologische Untersuchung.

Im Rahmen einer Pilotstudie wurde ein spezifisches Protokoll für die Untersuchung von Patienten mit dem Verdacht auf ein BFCS vorgeschlagen (Bodkin et al. 2009). Dabei soll mittels der repetitiven Stimulation des $\mathrm{N}$. tibialis durch schrittweise steigende Frequenzen $(2,5,10,20,50$ und $100 \mathrm{~Hz})$ das Auftreten von Nachtentladungen und deren Dauer, die kontinuierliche Aktivität der motorischen Einheit und Muskelkrampfpotentiale beobachtet werden (Liewluck et al. 2014). Unter Nachentladungen versteht man sich wiederholende oder anhaltende Entladungen eines induzierten Aktionspotentials entlang eines Nerven, die sofort auftreten und auch nach Beendigung des Reizes fortbestehen (Liewluck et al. 2014). Sie sind ein Ausdruck der Übererregbarkeit des Nerven bei diesen Patienten und es handelt sich dabei um Potentiale von motorischen Einheiten (Tahmoush et al. 1991, Harrison und Benatar 2007). Wenn die Frequenz des Reizes steigt, steigt auch die Anzahl der Nachtentladungen, und die Zeit, in der sie auftreten, wird ebenfalls länger (Tahmoush et al. 1991). Die meisten elektrischen Potentiale konnten dabei nach Reizung des N. peronaeus und des $\mathrm{N}$. tibialis posterior beobachtet werden, was den Erwartungen gerecht wurde, da die meisten Muskelkrämpfe im Bereich der Beine und Füße auftreten (Tahmoush et al. 1991). Harrison und Benatar konnten mit Hilfe dieser Untersuchung $75 \%$ der Patienten mit einem BFCS richtig identifizieren (Harrison und Benatar 2007). Durch die geringe Spezifität und das Fehlen von Referenzwerten bei gesunden Individuen ist ihre diagnostische Aussagekraft allerdings limitiert (Verdru et al. 1992, Liewluck et al. 2014).

Laboruntersuchungen zeigen außer einer evtl. leicht erhöhten Kreatinkinase keine spezifischen Auffälligkeiten (Tahmoush et al. 1991). Ein Augenmerk sollte insbesondere auf die Schilddrüsenhormone gelegt werden, da sowohl eine hyper- als auch eine hypothyreote Stoffwechsellage Faszikulationen und Muskelkrämpfe auslösen kann und diese Erkrankungen wichtige und behandelbare Differentialdiagnosen des BFCS darstellen (Hufschmidt und Lücking 2009). 
Die Diagnose eines BFCS wird aktuell anhand der Klinik gestellt, wenn alle weiteren Untersuchungen wie Elektrophysiologie, Labor und evtl. Muskelbiospie keinen Hinweis auf eine andere Genese der Beschwerden vermuten lassen.

\subsection{Pathophysiologie von Faszikulationen und Krämpfen}

\subsubsection{Haben Muskelkrämpfe einen zentralen oder peripheren Ursprung?}

Bis heute ist noch nicht vollständig geklärt, ob Muskelkrämpfe im Nervensystem vor allem einen zentralen oder peripheren Ursprung haben. Es werden zwei verschiedene Theorien favorisiert: (1) Muskelkrämpfe entstehen durch periphere Erregung von Motoneuronen, und spinale Mechanismen spielen nur eine untergeordnete Rolle, oder (2) spinale Mechanismen sind essentiell für die Entstehung und Aufrechterhaltung von Krämpfen (Minetto et al. 2011).

Für die erste Theorie spricht, dass es möglich ist, einen Muskelkrampf durch eine tetanische elektrische Stimulation eines peripheren Nerven auszulösen, auch wenn ein kompletter Leitungsblock z.B. durch Lidocain besteht (Tahmoush et al. 1991, Desai und Swash 1997, Miller und Layzer 2005). Allerdings ist die Reizschwelle zur Auslösung eines Krampfes ohne Leitungsblock geringer und die Dauer des elektrisch induzierten Muskelkrampfes länger, was für eine zentrale Modulation spricht (Minetto et al. 2011). Mithilfe des EMGs konnte gezeigt werden, dass Muskelkrämpfe durch Faszikulationen eingeleitet und beendet werden und da Faszikulationen einen peripheren Ursprung haben, spricht diese Beobachtung auch für eine distale Genese der Muskelkrämpfe (Layzer 1994, Lopate et al. 2013). Des Weiteren kann die Entladungsrate des Nerven während eines Muskelkrampfes mit bis zu $150 \mathrm{~Hz}$ sehr hoch sein, und es wird davon ausgegangen, dass zentrale Mechanismen Motoneurone nicht zu einer Entladungsrate über $50 \mathrm{~Hz}$ stimulieren können (Miller und Layzer 2005).

Die zweite Theorie (zentraler Ursprung) beruht auf der Annahme, dass Muskelkrämpfe durch eine Übererregbarkeit von Motoneuronen entstehen, die durch einen afferenten Input des zentralen Nervensystems hervorgerufen wird und daraus eine Art sich selbsterhaltender Regelkreis entsteht (Minetto et al. 2011). Hierfür spricht, dass sowohl ein EMG während eines Muskelkrampfes als auch eines während einer Willkürinnervation 
durch die Stimulation der afferenten Sehne inhibiert wird (Khan und Burne 2007). Außerdem bewirkt die aktive Anspannung des muskulären Antagonisten zum Muskel, der von dem Krampf betroffen ist, eine reziproke Hemmung oder einen cross-reflex-Effekt, die das EMG verändern, welches während eines Krampfes aufgezeichnet wird (Minetto et al. 2011).

Es wird deutlich, dass es für beide Theorien gute Argumente gibt und deswegen ist es noch nicht möglich, sich für eine dieser zwei Möglichkeiten zu entscheiden.

\subsubsection{Autoimmunologische Theorien}

Eine Theorie zum Ursprung des BFCS geht von einem immunologischen Geschehen aus. So sollen neuronale Autoantikörper die Ursache für Faszikulationen und Muskelkrämpfe sein (Hart et al. 2002, Liewluck et al. 2014). Es zeigte sich in beiden Studien, dass etwa ein Viertel der Patienten mit einem BFCS Antikörper gegen VGKC ausgebildet hatten (Hart et al. 2002, Liewluck et al. 2014). Allerdings fiel auf, dass die Patienten, die Autoantikörper ausgebildet hatten, signifikant öfter eine Übererregbarkeit des zentralen oder autonomen Nervensystems zeigten wie Benommenheit, vermehrtes Schwitzen (Hyperhydrosis) oder Epilepsie, wohingegen keiner der Patienten ohne Antikörper über solche Symptome klagte (Liewluck et al. 2014). Zu bedenken ist auch ein paraneoplastischer Ursprung der Antikörper, da einige Patienten Jahre vor der Diagnose eines Tumors Symptome der peripheren neuronalen Übererregbarkeit zeigten (Hart et al. 2002).

Das Vorliegen von Antikörpern gegen VGKC wurde erstmals im Zusammenhang mit dem Isaac- (Symptome: erworbene Neuromyotonie und Hyperhydrosis) und dem MorvanSyndrom (Symptome: limbische Enzephalitis, Neuromyotonie, Insomnie und autonome Dysfunktion) beschrieben (Klein et al. 2013). Allerdings ist die komplett ausgeprägte klinische Manifestation dieser Syndrome sehr selten und so scheint es ein fließendes Spektrum zwischen BFCS, Isaac- und Morvan Syndrom zu geben (Liewluck et al. 2014). Demnach sollten Patienten sehr genau auf zusätzliche Symptome des autonomen und zentralen Nervensystems untersucht werden, um die richtige Diagnose zu stellen und in dem Zusammenhang die richtige Therapie zu wählen.

Nichtsdestoweniger bleibt bei mindestens $75 \%$ der Patienten mit einem BFCS die Pathogenese der Symptome unklar. 


\subsection{Benignes Syndrom?}

Die alleinige Kombination aus Faszikulationen und Muskelkrämpfen ohne weitere klinische Auffälligkeiten wurde als benigne beschrieben, da sie ohne Muskelschwäche oder Atrophie einhergeht und nicht progredient ist (de Carvalho und Swash 2004, 2011). Allerdings zeigen, retrospektiv betrachtet, viele Patienten mit einer amyotrophen Lateralsklerose (ALS) als erste Symptome Muskelkrämpfe und Faszikulationen (de Carvalho und Swash 2004). Deshalb wurden 121 Patienten mit benignen Faszikulationen ohne Muskelschwäche, Atrophie und einem normalen EMG-Befund zwischen 2 und 32 Jahre nach der Diagnose erneut untersucht und keiner hatte in dieser Zeit eine ALS entwickelt (Blexrud et al. 1993, de Carvalho und Swash 2004). In den letzten Jahren haben sich allerdings die Berichte über Patienten gehäuft, die zunächst mit einer BFCS diagnostiziert wurden und später eine ALS entwickelten (Okuda et al. 1997, de Carvalho und Swash 2004, 2011, Singh et al. 2011).

Daher sollte die Diagnose eines BFCS nicht leichtfertig gestellt werden und sie scheint erst nach vier bis fünf Jahren ohne Progress zu einer Motoneuronerkrankung wirklich sicher zu sein (Singh et al. 2011).

\subsection{Therapie}

Die Leitlinien der Deutschen Gesellschaft für Neurologie empfehlen zur Behandlung von Muskelkrämpfen allgemein die Dehnung der betroffenen Muskelgruppen bei Krämpfen, die belastungsabhängig oder überwiegend nachts auftreten, und die Gabe von 200-400 mg Chininsulfat oder Hydrochinin zur Nacht bei sehr schmerzhaften und häufigen Krämpfen, die nicht durch eine behandelbare Ursache entstehen (AWMF 2012). Allerdings sollten die seltenen, aber schweren Nebenwirkungen der Chinine wie Thrombozytopenien (Park et al. 2009) und die Arzneimittelinteraktionen beachtet werden (Bateman und Dyson 1986). Chinin scheint eine gute Wirksamkeit auf Krämpfe in den Beinen zu haben (Man-Son-Hing et al. 1998), allerdings fehlen Daten über die Wirksamkeit beim BFCS.

Viele Patienten mit einem BFCS scheinen gut auf die Therapie mit membranstabilisierenden Medikamenten wie Carbamazepin, Gabapentin und Pregabalin anzusprechen (Tahmoush et al. 1991, Liewluck et al. 2014). Tahmoush und Kollegen testeten 
die Wirkung von Carbamazepin bei neun Patienten mit einem BFCS, wobei fünf sehr gut auf die Therapie ansprachen, einer gut und drei zeigten nur eine mäßige Besserung der Symptome (Tahmoush et al. 1991).

In einem open-label-Versuch mit 30 Patienten, die öfter als fünf Mal pro Woche an Muskelkrämpfen litten, konnte gezeigt werden, dass nach einer Behandlungsdauer von drei Monaten mit einer Dosis von 600-1200 mg Gabapentin pro Tag sich die Beschwerden bei allen Teilnehmern deutlich gebessert hatten (Serrao et al. 2000).

Größere Studien zur Effektivität dieser Medikamente bei Muskelkrämpfen und insbesondere beim BFCS fehlen noch.

\subsection{Physiologische Bedeutung der HCN-Kanäle}

\subsubsection{Aufbau und Regulierung}

HCN-Kanäle sind eine Subgruppe der Kationen-Kanäle, die durch zyklisches Nukleotid reguliert werden und gehören zu der Gruppe der Kanäle, die durch Transmembranproteine gebildet werden und eine ionendurchlässige Pore besitzen (Wahl-Schott und Biel 2009). Sie bestehen aus vier Untereinheiten, die um die zentrale Pore arrangiert sind (s. Abb. 1A) Jede Untereinheit besteht aus sechs Segmenten (S1-S6), wobei sich die Pore zwischen S5 und S6 befindet, S4 den Spannungssensor bildet und die Gylcin-Tyrosin-Glycin (GTG)Sequenz den Selektivitätsfilter darstellt (s. Abb.1B) (Wahl-Schott und Biel 2009). Vier Isoformen der HCN-Kanäle (HCN 1-4) wurden an peripheren Nerven von Säugetieren entdeckt, die sich in ihrer Funktion und Expression in verschiedenen Gewebetypen unterscheiden und das molekulare Korrelat des hyperpolarisationsaktivierten Stroms $\left(I_{H}\right)$ bilden (Chaplan et al. 2003, Doan et al. 2004, Hofmann et al. 2005, Biel et al. 2009, Trevillion et al. 2010). In sensiblen Fasern wurde eine vermehrte Expression von HCN1 und HCN2 gefunden (Moosmang et al. 2001). Allerdings wurde die Expression der einzelnen Isoformen an humanen sensiblen und motorischen peripheren Axonen bis jetzt noch nicht belegt.

$\mathrm{HCN}-\mathrm{Kanäle}$ sind permeabel für Natrium $\left(\mathrm{Na}^{+}\right)$und $\mathrm{K}^{+}$in einem Verhältnis von 1:4 und können durch geringere Konzentration von Caesium (Cs+) blockiert werden (Pape 1996, Wahl-Schott und Biel 2009). Unter physiologischen Bedingungen ist die Flussrichtung der 
Ionen sowohl für $\mathrm{K}^{+}$als auch für $\mathrm{Na}^{+}$nach innen gerichtet und auch für Kalzium $\left(\mathrm{Ca}^{2+}\right)$ scheint eine geringe Permeabilität zu bestehen, wobei die Funktion des $\mathrm{Ca}^{2+}$-Stroms noch unklar ist (Yu et al. 2004, Wahl-Schott und Biel 2009).

$I_{H}$ wird, im Gegensatz zu allen anderen spannungsabhängigen lonenkanälen, durch hyperpolarisierende Potentiale ab $-55 \mathrm{mV}$ aktiviert, welches somit in der Nähe des Ruhemembranpotentials der meisten Zellen mit etwa $-70 \mathrm{mV}$ bis $-90 \mathrm{mV}$ liegt und ist demzufolge auch an der Modulation eben dieses Ruhemembranpotentials beteiligt (Biel et al. 2009). Die HCN-Kanäle sind vor allem spannungsabhängig, allerdings scheint auch die Modulation durch zyklisches Adenosinmonophosphat (cAMP) eine Rolle zu spielen, welches direkt an diese Kanäle über eine Bindungsstelle (cyclic nucleotide-binding domain $=$ CNBD) zu binden und nicht über die Beeinflussung einer Proteinkinase zu wirken scheint (s. Abb. 1C) (Wahl-Schott und Biel 2009). Durch cAMP werden Kanäle so beeinflusst, dass sie zum einen schneller und zum anderen bereits bei weniger negativen Potentialen öffnen und demnach eine Veränderung ihrer Spannungsabhängigkeit herbeigeführt wird (DiFrancesco und Tortora 1991, Biel et al. 2009). Die CNBD befindet sich am C-terminalen Ende des Proteins, welches ein Monomer des HCN-Kanals bildet und wird über einen sogenannten C-Linker mit dem Segment S6 verbunden (s. Abb. 1C) (Wahl-Schott und Biel 2009).

A

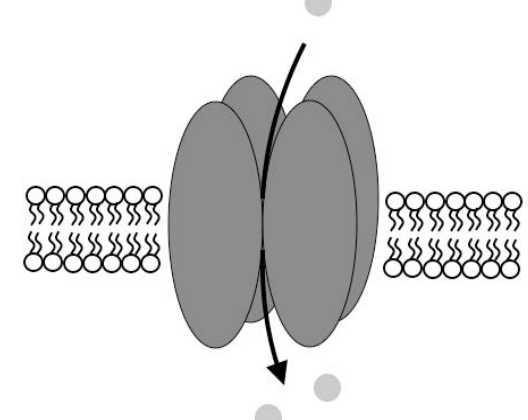

B

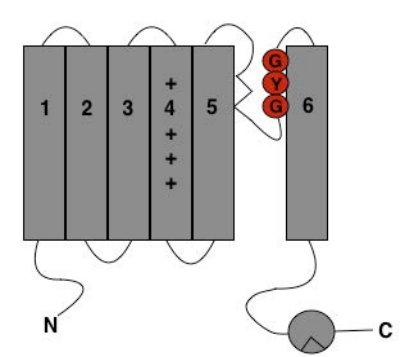

C

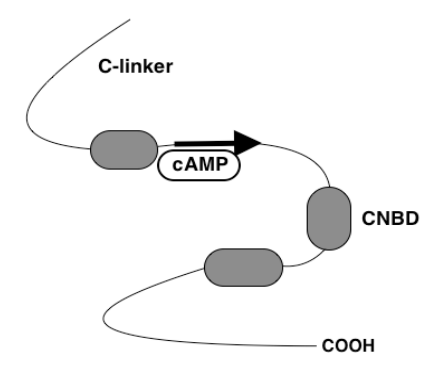

(modifiziert nach Wahl-Schott und Biel 2009)

Abbildung 1 (A) Aufbau des HCN-Kanals als Tetramer mit (B) = ein Monomer bestehend aus sechs transmembranären Segmenten (S1-S6), dem Spannungssensor (S4), dem Selektivitätsfilter (GYG) und der Pore zwischen S5 und S6. N-terminales und $(C)=C$-terminales Ende bestehend wiederum aus zwei Untereinheiten: C-Linker und der Bindungsstelle für CAMP (CNBD) 
Abgesehen von cAMP gibt es noch viele weitere Regulierungsmechanismen für $\mathrm{HCN}$ Kanäle. Phosphatidylinositol-4,5-bisphosphat $\left(\mathrm{PIP}_{2}\right)$ verschiebt als allosterischer Ligand die spannungsabhängige Aktivierung der $\mathrm{HCN}$-Kanäle um ca. $20 \mathrm{mV}$ zu positiveren Potentialen (Wahl-Schott und Biel 2009). Des Weiteren scheint die Protonenkonzentration eine Rolle für die Aktivität der HCN-Kanäle zu spielen. Intrazelluläre Protonen verschieben dabei die Aktivierung Richtung mehr hyperpolarisierten Potentialen und führen zu einer langsameren Öffnung, wohingegen ein niedriger extrazellulärer $\mathrm{pH}$ die Spannungsabhängigkeit so moduliert, dass die Kanäle bereits bei positiveren Potentialen öffnen (Munsch und Pape 1999, Stevens et al. 2001, Zong et al. 2001). Darüber hinaus scheinen noch die extrazelluläre Chloridkonzentration, Tyrosinphosphorylierung, die p38-MAP (mitogen-activated-protein)-Kinase und die Regulierung über interagierende Proteine eine Rolle zu spielen (Wahl-Schott und Biel 2009).

\subsubsection{Funktion an peripheren Nerven}

Die physiologische Rolle des $\mathrm{I}_{\mathrm{H}}$-Stroms in Nervenzellen scheint vor allem in der Regulierung des Ruhemembranpotentials und in einer Art negativem Feed-backMechanismus zu liegen, da die Kanäle sowohl einer Membrandepolarisation als auch einer -hyperpolarisation entgegenwirken können (Biel et al. 2009). Eine Hyperpolarisation der Membran bewirkt, dass mehr HCN-Kanäle aktiv sind, die zu einer Depolarisation der Membran führen, wohingegen eine Depolarisation der Membran zum Schließen der Kanäle, die während des Ruhemembranpotentials aktiv sind, führt und somit indirekt eine Hyperpolarisation bewirkt wird (Biel et al. 2009). Durch diesen Mechanismus sind die HCN-Kanäle an der Regulierung der Exzitabilität der Nerven beteiligt und, da sie nicht inaktivieren, vermindern sie gerade bei Membranhyperpolarisation den Widerstand für eintreffende Impulse und verhindern so die Entstehung eines Leitungsblocks (Krishnan et al. 2009) . 


\subsubsection{Funktion am Herzen}

Die Funktion der HCN-Kanäle ist am besten am Herzen untersucht worden. Hier wird der Strom durch die HCN-Kanäle als $\mathrm{I}_{\mathrm{f}}$-Strom ( $\mathrm{f}=$ funny) bezeichnet und kontrolliert die Herzfrequenz und den Rhythmus, indem er als Schrittmacher-Strom (= pacemaker current) fungiert (Stieber et al. 2003, Hofmann et al. 2005, Scicchitano et al. 2012, Kuwabara et al. 2013). Veränderungen dieser Kanäle können im Herzen zu lebensbedrohlicher kardialer Dysfunktion und Arrhythmien führen (Kuwahara et al. 2003, Dias und Terracciano 2013).

Es wird deutlich, wie wichtig es ist, die Funktion dieser Kanäle richtig zu verstehen um dieses Wissen dann auch therapeutisch zu nutzen.

\subsection{Einführung in das Threshold tracking}

\subsubsection{Entwicklung des Threshold trackings}

Die Methode hat ihren Ursprung 1970, als Joseph Bergmans zum ersten Mal den Nutzen des Messens von Schwellenwerten an menschlichen Motoaxonen erkannte, da er anhand der Veränderungen des Schwellenwertes durch Reizweiterleitung und polarisierende Pulse Informationen über die Physiologie des Axons gewinnen konnte (Bostock et al. 1998). Dazu musste allerdings sehr aufwendig eine einzelne funktionierende Nervenfaser isoliert und dann der Schwellenwert dieser Faser manuell festgelegt werden (Bostock et al. 1998). Das Problem des manuellen Festlegens des Schwellenwertes wurde zunächst durch einen sogenanten threshold hunter (Raymond 1979) und später durch einen threshold tracker gelöst, der die Amplitude des Stroms veränderte anstelle der Dauer, wie es der threshold hunter vorgenommen hatte (Bostock et al. 1983). Da sich der Schwellenwert eines Summenaktionspotential genauso verhält, wie das Potential einer einzelnen Faser, konnte das Isolieren der einzelnen Faser umgangen werden und das threshold tracking wurde für den klinischen Gebrauch leichter durchführbar (Bostock et al. 1998).

Heute wird das threshold tracking mithilfe eines Computers und einer threshold-trackingSoftware automatisch durchgeführt, sodass innerhalb einer kurzen Zeit von 10 bis 15 Minuten alle wichtigen Exzitabilitätsparameter gemessen werden können, die Methode 
sehr einfach durchführbar ist und die Daten reproduzierbar sind (Kiernan et al. 2000, Burke et al. 2001). Dabei wird nicht nur der Schwellenwert des Axons bestimmt, sondern vor allem untersucht, wie sich dieser durch Abgabe von über- und unterschwelligen Reizen verändert (Bostock et al. 1998). Durch die Abgabe dieser Reize nach einem spezifischen Protokoll können die wichtigsten Exzitabilitätsparameter des Nerven errechnet werden. Vor Kurzem wurde dieses Protokoll noch durch stärker hyperpolarisierende Impulse erweitert um die Funktion der HCN-Kanäle am peripheren Nerven besser untersuchen zu können (Tomlinson et al. 2010a).

Das threshold tracking findet aktuell vor allem Anwendung in der Forschung um physiologische und pathophysiologische Mechanismen der axonalen Erregbarkeit zu klären.

\subsubsection{Threshold tracking im Vergleich zur neurophysiologischen Routinediagnostik}

Die neurophysiologischen Routinediagnostik der Funktion des Nerven fokussiert sich auf die Messung der NLG und die Anzahl der weiterleitenden Fasern (Bostock et al. 1998, Burke et al. 2001). Diese Tests geben Aufschluss über den Zustand der Myelinscheide, aber können nur wenige Informationen über die Membran des Axons liefern (Bostock et al. 1998). Somit werden die Veränderungen der axonalen Erregbarkeit, die durch einen Leitungsblock oder ektopische Reize entstehen in der Routinediagnostik nicht erfasst (Burke et al. 2001).

Das threshold tracking hingegen testet die Erregbarkeit des Nerven, welche direkt von den Eigenschaften der Membran abhängen, und ist somit sensitiv für die Messung des Membranpotentials und Veränderungen eben dieses durch die Aktivierung von Ionenkanälen und -pumpen auch unter der Myelinscheide während und nach der Weiterleitung von Impulsen oder durch unterschwellige Reize (Bostock et al. 1998). Allerdings wird die Erregbarkeit des Nerven nur an dem Punkt, an dem der Nerv stimuliert wird, getestet und damit ist das threshold tracking für fokale Geschehen nur eingeschränkt verwendbar (Bostock et al. 1998). Wichtig ist, dass die verschiedenen Exzitabilitätsparameter, die das threshold tracking misst, immer im Zusammenhang zueinander gesehen werden müssen, da die Veränderungen eines Parameters viele verschiedene 
Ursachen haben kann (Kiernan et al. 2000).

Mittlerweile ist es auch möglich mithilfe dieser Technik sensorische Fasern zu untersuchen, wodurch periphere Nervenerkrankungen, die vorwiegend sensorische Symptome zeigen, untersucht werden können (Kiernan et al. 2000).

Das threshold tracking ist es eine hervorragende Methode um in relativ kurzer Zeit Informationen über die Erregbarkeit von Nerven in vivo zu gewinnen und es bietet eine große Chance die zugrundeliegenden Pathologien von peripheren Nervenerkrankungen näher zu charakterisieren.

\subsection{Zielsetzung}

In dieser Arbeit sollen die Exzitabilitätsparameter von Patienten, die unter einem BFCS leiden, mit einer gesunden Kontrollgruppe verglichen werden. Dadurch sollen mehr Informationen über den zugrundeliegenden Pathomechanismus gewonnen werden, der bis heute noch nicht vollständig geklärt ist.

Zum ersten Mal wird eine größere Anzahl dieser Patienten mit Hilfe des threshold trackings untersucht. Die Messung wird anhand des erweiterten Trond-Protokolls durchgeführt (Bostock et al. 1998, Kiernan et al. 2000, 2001a). Das Protokoll umfasst somit auch stärker hyperpolarisierende konditionierende Impulse. Dadurch wird es möglich, die Funktion der HCN-Kanäle genauer zu untersuchen. 


\section{Material und Methoden}

\subsection{Probandenkollektiv und Studiendesign}

Im Rahmen der Arbeit wurden insgesamt 33 Probanden untersucht, 18 (13 weibliche und 5 männliche) Probanden, die an einem BFCS litten, und 15 (6 weibliche und 9 männliche) gesunde Probanden als Kontrollgruppe. Alle Patienten mit einem BFCS, die an dieser offenen neurophysiologischen klinischen Studie teilnahmen, wurden zwischen Mai 2012 und Januar 2014 mittels Aushängen rekrutiert und untersucht oder wurden durch niedergelassene Neurologen auf die Studie aufmerksam gemacht. Die gesunden Probanden wurden mittels Aushängen für diese Studie gewonnen. Einschlusskriterien der Studie waren ein Alter zwischen 18 und 80 Jahren. Ausschlusskriterien waren ein Herzschrittmacher, tiefe Hirnstimulation, Vorliegen einer gravierenden internistischen oder psychiatrischen Vorerkrankung, Schwangerschaft und Stillzeit, Alkohol-, Medikamentenund Drogenabhängigkeit, rezeptive oder globale Aphasie sowie Vorliegen einer gesetzlichen Betreuung.

Die Probanden wurden hinsichtlich Sensibilität, Motorik und Reflexstatus klinisch untersucht und es ergaben sich keine Auffälligkeiten. Die Messung fand im Sitzen statt, wobei die Probanden ihren Arm einfach entspannt ablegen und während der Messung nicht bewegen sollten. In den meisten Fällen wurde der rechte N. medianus gemessen, außer wenn es sich bei dem Probanden um einen Linkshänder handelte oder andere Probleme an der rechten Hand vorlagen, wie zum Beispiel ein Karpal-Tunnel-Syndrom, welches nur den rechten $\mathrm{N}$. medianus betraf.

Alle Probanden wurden über Zweck, Ziel und mögliche Risiken der Studie aufgeklärt und gaben ihr schriftliches Einverständnis. Das Studienprotokoll wurde von der Ethikkommission der Universität Göttingen geprüft und akzeptiert (Antragsnummer 20/2/12) und entspricht der Deklaration von Helsinki. 


\subsection{Threshold tracking}

Das threshold tracking ist eine Methode, bei der es darum geht, immer den konstanten Teil eines Summenaktionspotentials eines Muskels, der als Schwellenwert (= threshold) definiert wird, durch Anpassung der Reizintensität zu erreichen, da durch konditionierende Vorimpulse das Membranpotential verändert wird und somit eine andere Reizintensität benötigt wird, um dieselbe Antwort zu erzeugen.

Das threshold tracking wurde durch einen Computer mit dem Programm QtracS (@) Professor H. Bostock, Institute of Neurology, London, Version 10/5/2011) gemäß dem Trond- Protokoll (Bostock et al., 1998; Kiernan et al., 2000, 2001a) durchgeführt. Der N. medianus wurde mittels eines isolierten bipolaren Gleichstromstimulators (Digitimer DS5 bipolar constant current stimulator, Digitimer Ltd, UK) (max. +/- $50 \mathrm{~mA}$ ) am Handgelenk stimuliert. Als Elektroden wurden Asmuth EKG-Elektroden (MPC International S.A., Luxemburg) verwendet.

Die Stimulationselektrode (Kathode) wurde über dem N. medianus am Handgelenk angebracht und die Referenzelektrode (Anode) ca. $10 \mathrm{~cm}$ proximal davon am Unterarm, sodass sie nicht über dem proximalen Verlauf des $\mathrm{N}$. medianus lag. Das Summenaktionspotential des Muskels wurde vom $\mathrm{M}$. abductor pollicis brevis abgeleitet, indem die Ableitungselektrode (Kathode) auf den Muskel und die Referenzelektrode (Anode) der Ableitung auf die proximale Phalanx des Daumens geklebt wurde. Das abgeleitete Potential wurde dann durch den D360 Digitimer (UK) vorverstärkt. Die Erdungselektrode wurde palmar zwischen der Basis des kleinen Fingers und des Ringfingers angebracht (Anordung der Elektroden: s. Abb. 2). Das elektromyographische Signal wurde mittels eines Computers (Dell Optiplex 745) und eines Analog-zu Digital-Umwandlers (NI USB6251, National Instruments) digitalisiert. Durch einen Noise Eliminator wurde das Signal verbessert (Quest Scientific, Hum Bug 50/60 Hz Noise Eliminator). Während der gesamten Messung betrug die Hauttemperatur $>32^{\circ} \mathrm{C}$, was mit einer Temperatursonde (EcoScan Temp4 Thermistar Thermometer von Eutech Instruments) nahe der Stimulationselektrode kontrolliert wurde. Wenn die Temperatur vor der Messung unter $32^{\circ} \mathrm{C}$ betrug, wurde sie mittels Decken oder einer Wärmelampe angehoben. Die Haut wurde mittels eines Schleifpapiers und alkoholischem Desinfektionsmittel vorbereitet, um die Leitfähigkeit zu erhöhen. 


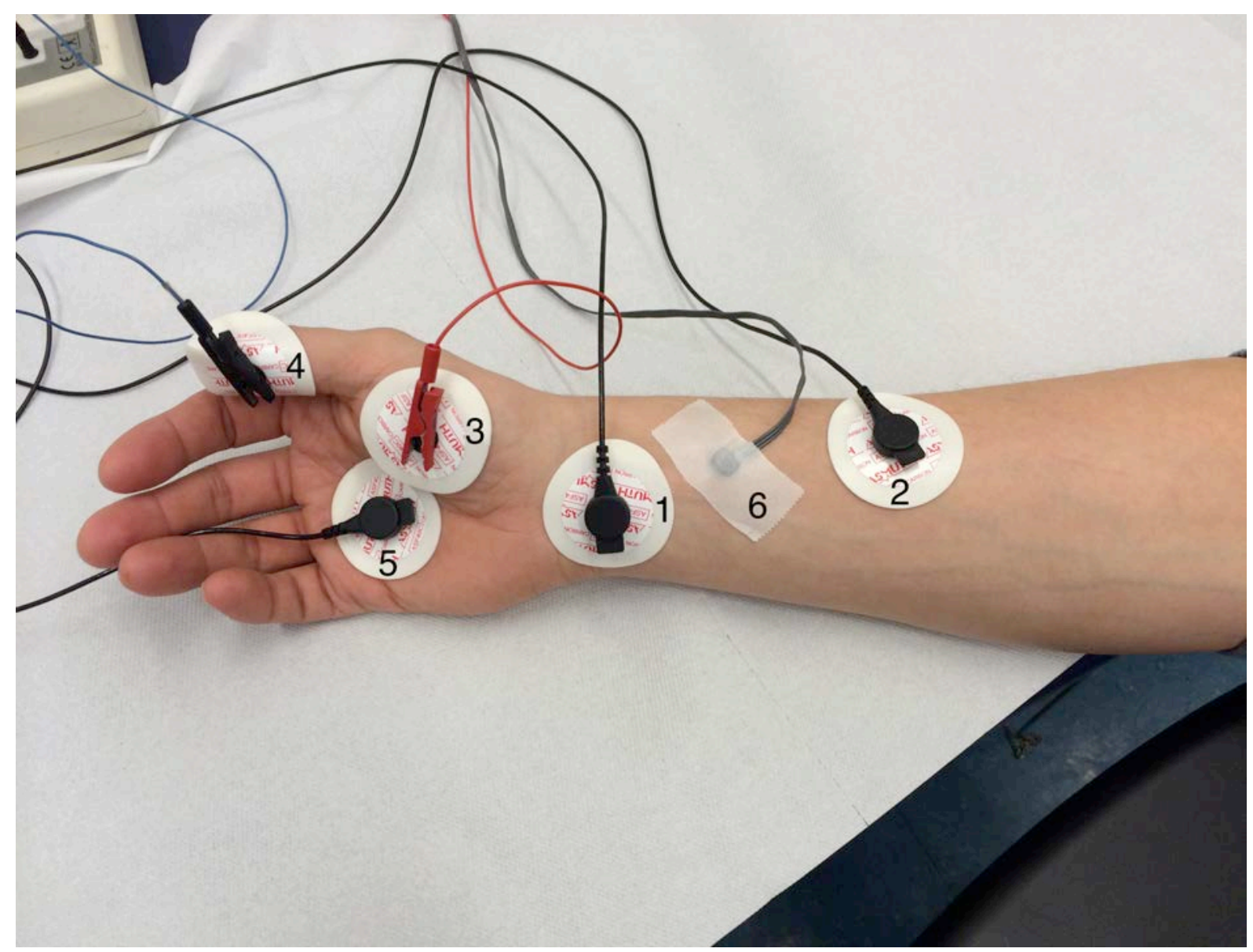

Abbildung 2 Anordnung der Elektroden zur Messung des rechten N. medianus mit 1 = Stimulationselektrode (Kathode), 2 = Referenzelektrode zu 1 (Anode), 3 = Ableitungselektrode (Kathode), 4 = Referenzelektrode zu 3 (Anode), 5 = Erdungselektrode, $6=$ Temperatursonde

\subsection{Trond-Protokoll}

Das Trond-Protokoll beinhaltet die Messung vieler Exzitabilitätsparameter. Im Folgenden werden die Messung und Bedeutung der einzelnen Parameter genauer erläutert.

\subsubsection{Stimulus-response-curve}

Die stimulus-response-curve wurde erzeugt, indem ein 1 ms langer Testimpuls kontinuierlich gesteigert wurde, bis ein maximales Summenaktionspotential des Muskels erreicht werden konnte, das auch bei weiterer Impulssteigerung nicht mehr zunahm. Die Stärke des Reizes nahm dabei jeweils um 2\% zu. Das Programm reduzierte die Reizintensität dann langsam wieder, bis die Antwort auf null abfiel. Der Zielwert für das threshold 
tracking wurde automatisch bei $40 \%$ der maximalen Amplitude festgesetzt. Dieser Wert wurde dann als Schwellenwert für Motoaxone definiert. Während der einzelnen Messungen wurde der Impuls, der nötig ist, um den Schwellenwert zu erreichen, als Kontrollwert herangezogen (1 ms Reiz, keine konditionierenden Reize), um ihn mit den Reizintensitäten nach konditionierenden Reizen zu vergleichen. Durch verschiedene Vorimpulse musste die Reizintensität verändert werden, um weiterhin $40 \%$ des maximalen Summenaktionspotentials des Muskels zu erreichen. Die Anpassung des Testimpulses wurde dabei computerkontrolliert vorgenommen. Die Messung der stimulus-responsecurve ist beispielhaft in Abbildung 8 dargestellt.

\subsubsection{Strength-duration properties}

Der Kontrollimpuls wurde verglichen mit Reizen unterschiedlicher Dauer. Begonnen wurde mit einer Dauer von $1 \mathrm{~ms}$, danach nahm die Dauer kontinuierlich um 0,2 ms ab (1,0;0,8; 0,6;0,4;0,2 ms). Es wurde die Stärke des Reizes gemessen, die benötigt wurde, um den Kontrollschwellenwert $(=40 \%$ des maximalen Summenaktionspotentials) zu erreichen. Je länger der Reiz dauerte, desto weniger stark musste dieser sein, um die gleiche Antwort zu erzielen (Krishnan et al. 2009). Mittels des Weiss'schen Gesetzes können der strengthduration time constant (= konstanter Zeitwert der Beziehung zwischen Intensität und Dauer des Reizes) und die Rheobase abgeschätzt werden (Bostock et al. 1983, Mogyoros et al. 1996). Rheobase ist definiert als die Reizintensität, die benötigt wird, ein Aktionspotential auszulösen, wenn der Impuls unendlich lang wäre und Chronaxie als die Reizdauer, bei der die Reizintensität doppelt so stark ist wie bei der Rheobase (s. Abb. 3). Der strength-duration time constant beschreibt die lineare Beziehung zwischen elektrischer Ladung (mA $\times$ ms) und Reizdauer (Jankelowitz et al. 2007b) (s. Abb. 4). In peripheren Axonen ist der strength-duration time constant nach dem Weiss'schen Gesetz identisch mit der Chronaxie (Bostock 1983, Mogyoros et al. 1996, Krishnan et al. 2009). Der strength-duration time constant und die Rheobase sind Eigenschaften der Ranvier 'schen Schnürringe und verknüpft mit der $\mathrm{Na}^{+}$-Leitfähigkeit (Kiernan et al. 2000, Krishnan et al. 2009). Eine Steigerung des $\mathrm{Na}^{+}$-Stroms oder eine Depolarisation des Ranvier'schen Schnürringes würde den strength-duration time constant verlängern und die Rheobase verkleinern (Bostock und Rothwell 1997, Kiernan et al. 2000). Eine Messung der strengthduration properties ist in Abbildung 8 dargestellt. 


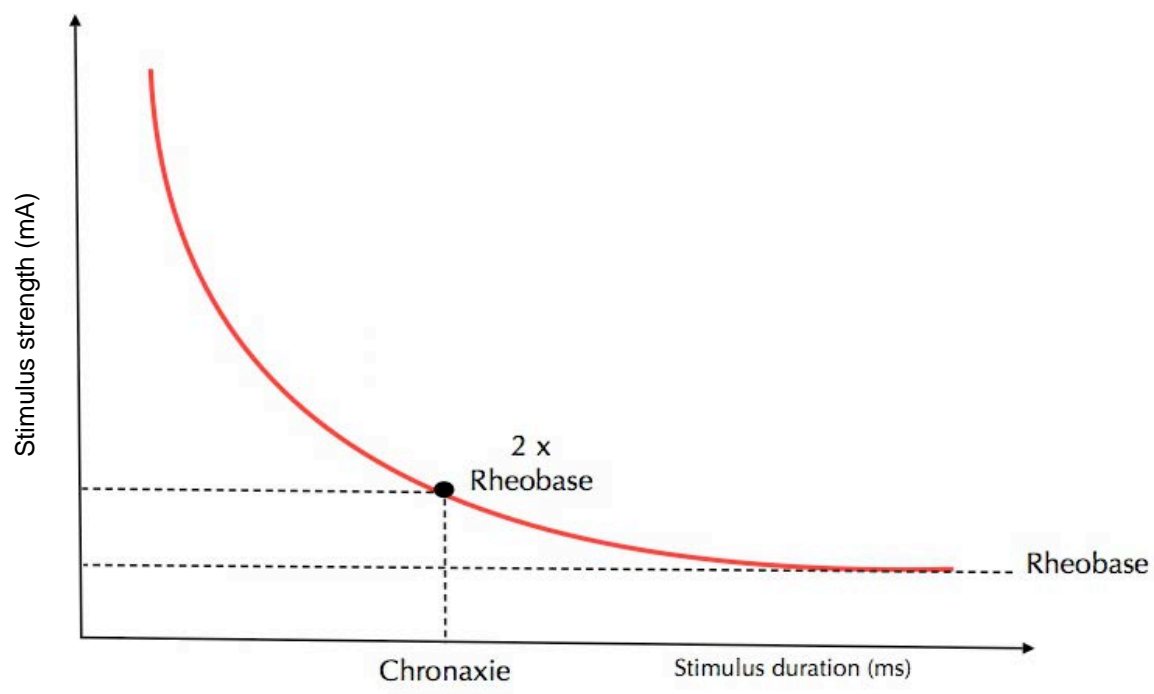

Abbildung 3 Aufgetragen ist die Reizintensität (= Stimulus strength in $m A$ ) auf der $y$-Achse gegen die Reizdauer (= Stimulus duration in $m s$ ) auf der $x$-Achse, um die Bedeutung von Rheobase und Chronaxie zu verdeutlichen: Rheobase = Reizintensität, die benötigt wird um ein Aktionspotential auszulösen, wenn der Reiz unendlich lang wäre; Chronaxie: Reizdauer, bei der die Reizintensität doppelt so stark ist wie bei der Rheobase (entspricht dem strength-duration time constant nach dem Weiss'schen Gesetz)

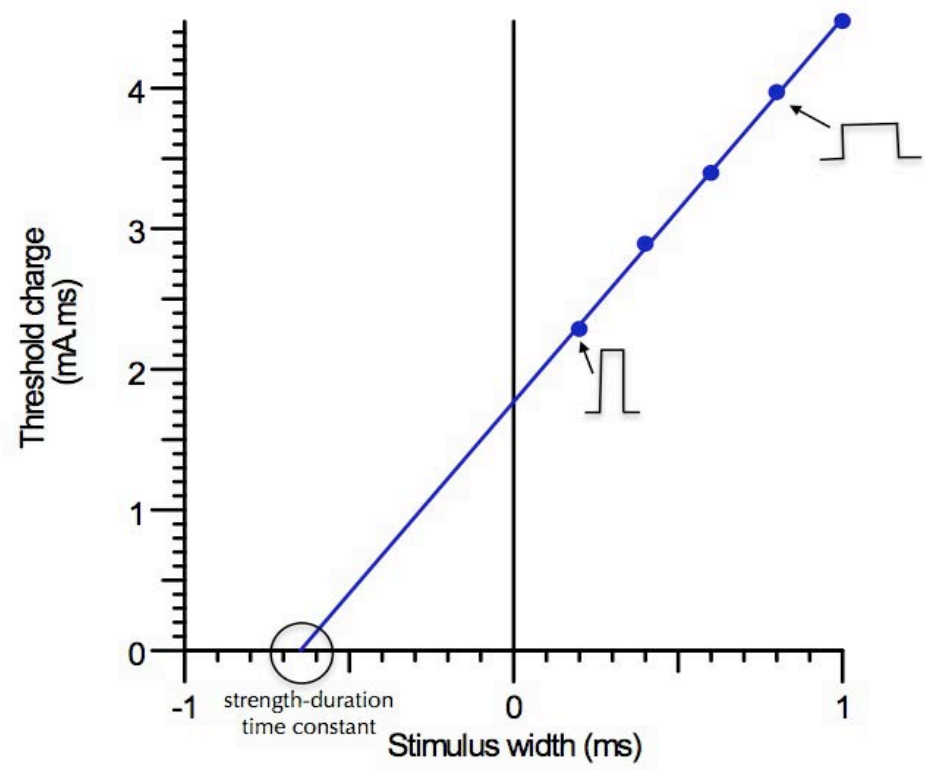

Abbildung 4 Strength-duration time constant nach dem Weiss'schen Gesetz: je länger der Reiz dauert, desto niedriger muss die Reizintensität sein. Wie hier gezeigt, kann der strength-duration time constant über den negativen Schnittpunkt der Regressionslinie auf der x-Achse bestimmt werden, indem die notwendige Reizintensität für vier unterschiedlich lange Reize gemessen wird. 


\subsubsection{Threshold electrotonus}

Der threshold electrotonus spiegelt die Veränderungen des Schwellenwertes wieder, die sich während und nach unterschwelligen konditionierenden Reizen ergeben. Somit stellt er die elektrischen Veränderungen des Membranpotentials des Nerven dar (Baker et al. 1987, Bostock und Baker 1988, Bostock et al. 1998, Krishnan et al. 2009).

Wie in Abbildung 5 zu sehen, zeigt sich nach depolarisiereden konditionierenden Reizen zunächst eine schnelle Phase, die proportional zu dem abgegeben Reiz ist (F-Phase) (Jankelowitz et al. 2007B, Krishnan et al. 2009). Es folgt eine langsamere Depolarisation, die maximal zwischen 15-20 ms lang ist und die fortschreitende Depolarisation abbildet, die sich langsam bis zur internodalen Membran ausbreitet (S1-Phase) (Baker et al. 1987, Bostock et al. 1998, Krishnan et al. 2009). Die Depolarisation nimmt daraufhin langsam wieder $\mathrm{ab}$, da sich langsame $\mathrm{K}^{+}-$Kanäle an den Ranvier'schen Schnürringen und den Internodien öffnen (S2-Phase) und nähert sich schließlich dem Kontrollschwellenwert an (Baker et al. 1987, Bostock und Baker 1988, Bostock et al. 1998, Krishnan et al. 2009). Am Ende steigt der Schwellenwert schnell an und übersteigt schließlich den Kontrollschwellenwert durch die internodale Polarisation und die allmähliche Deaktivierung der langsamen K+-Kanäle (undershoot) (Bostock und Rothwell 1997, Krishnan et al. 2009)

In hyperpolarisierender Richtung zeigt sich wiederum eine schnelle Phase, die proportional zu dem abgegeben Reiz ist (F-Phase). Gefolgt wird diese Phase von einer Hyperpolarisation, da durch das Schließen von internodalen $\mathrm{K}^{+}$-Kanälen der Membranwiderstand steigt (S1-Phase) (Jankelowitz et al. 2007b). Die S1-Phase ist hier deutlicher ausgeprägt als nach depolarisierenden Reizen, da zwar K+-Kanäle durch Hyperpolarisation geschlossen werden, aber das Ausmaß der Hyperpolarisation dadurch nicht vermindert werden kann (Bostock et al., 1998). Die Hyperpolarisation wird schließlich durch die Aktivierung des $\mathrm{I}_{\mathrm{H}}$-Stroms limitiert (S3-Phase) (Pape 1996, Bostock et al. 1998, Krishnan et al. 2009). Zum Schluss sinkt der Schwellenwert ab bis unter das Niveau des Kontrollschwellenwertes durch die Inaktivierung des $\mathrm{I}_{\mathrm{H}}$-Stroms und die Aktivierung von Kalium-Kanälen (overshoot) (Krishnan et al. 2009). 


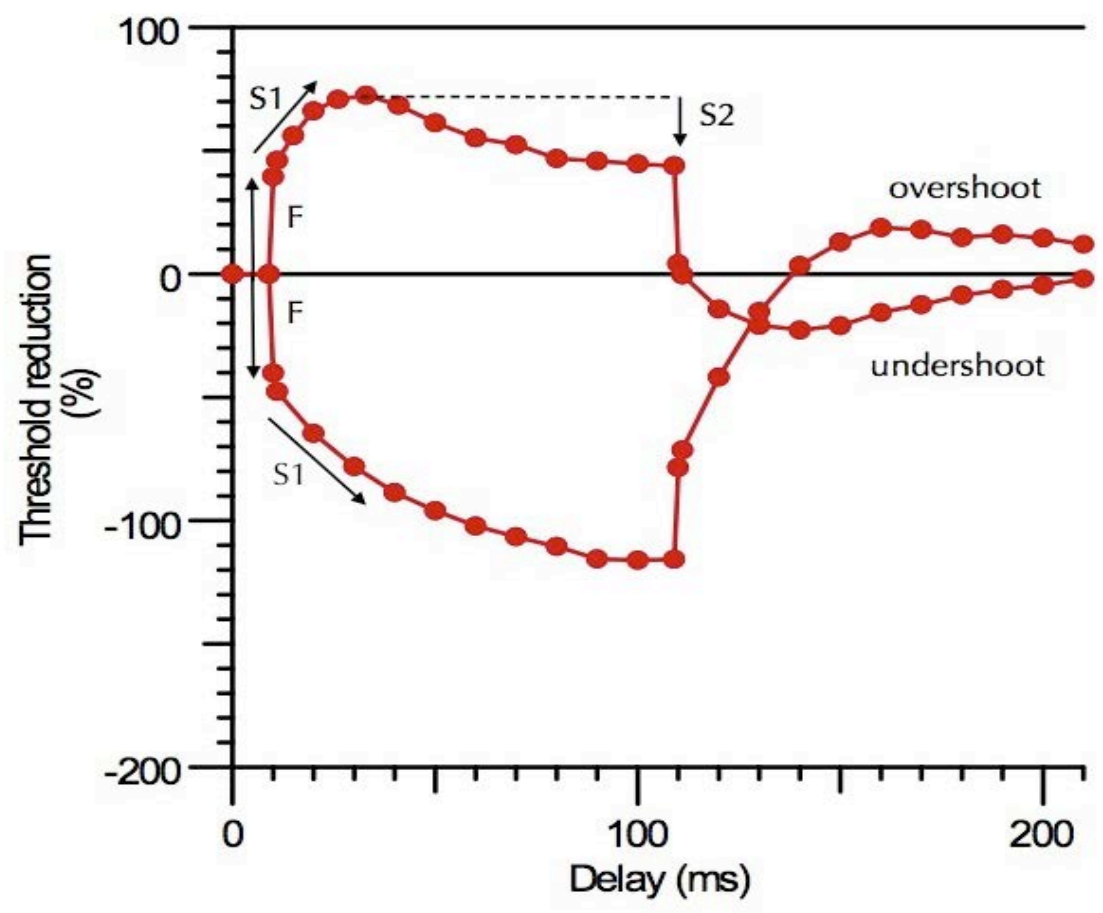

Abbildung 5 Phasen des threshold electrotonus nach einem 100 ms langen unterschwelligen polarisierenden Reiz ( $+40 \%$ des Kontrollimpulses nach oben und $-40 \%$ nach unten aufgetragen). Die erste schnelle (F) Phase als proportionale Antwort zum abgegebenen Reiz wird von einer langsameren (S1) Phase gefolgt, die durch die Ausbreitung des lonenstroms zu den Internodien entsteht. Die S2Phase in depolarisierender Richtung entsteht durch die Aktivierung langsamer $\mathrm{K}^{+}$-Kanäle. Der undershoot nach depolarisierenden Reizen entsteht durch die internodale Polarisation und die Dektivierung der langsamen $\mathrm{K}^{+}$-Kanäle. Nach hyperpolarisierenden Reizen kommt es zu einem overshoot durch durch die Inaktivierung des $I_{H^{-}}$Stroms und der Aktivierung von $K^{+}$-Kanälen.

Nach depolarisierenden Vorimpulsen kommt es zu einer Abnahme des Schwellenwertes (Burke et al. 2001, Tomlinson et al. 2010b). Je höher die Kurve nach der Depolarisation ist, desto niedriger ist der Schwellenwert. Bei den hyperpolarisierenden konditionierenden Reizen ist es genau umgekehrt: es kommt zu einem Anstieg des Schwellenwertes und somit bedeutet eine ausgedehntere Kurve, dass ein höherer Schwellenwert für das Auslösen eines Aktionspotentials vorliegt (Burke et al. 2001, Tomlinson et al. 2010b). 
Den Probanden wurden im Rahmen des threshold electrotonus unterschwellige konditionierende Reize appliziert, die sowohl de- als auch hyperpolarisierend waren. Die Reize wurden als ein bestimmter Anteil des Kontrollimpulses festgelegt: 100 ms lange Reize mit $+40 \%,+20 \%$, $-20 \%$ und $-40 \%, 200$ ms lange Reize mit $-70 \%$ und 300 ms lange Reize mit $-100 \%$ des Kontrollimpulses. Die Impulse wurden nacheinander abgeben: zunächst der $1 \mathrm{~ms}$ lange Testimpuls ohne konditionierenden Reiz um einen Kontrollwert zu erzeugen, dann der depolarisierende konditionierende Reiz + Testimpuls bzw. der hyperpolarisierende konditionierende Reiz + Testimpuls. Die Reizintensität, die benötigt wurde um den Zielwert des Summenaktionspotentials des Muskels zu erreichen, wurde zu verschiedenen Zeitpunkten mit einem maximalen Zeitabstand von $10 \mathrm{~ms}$ zwischen den Punkten gemessen und zwar vor, während und bis zu 300 ms nach den konditionierenden Reizen. Die Messung des threshold electrotonus ist beispielhaft in Abbildung 8 wiedergegeben.

\subsubsection{Current/threshold relationship (I/V)}

Vor den 1 ms langen Testimpulsen wurden 200 ms lange unterschwellige de- und hyperpolarisierende Vorimpulse abgeben. Begonnen wurde mit $+50 \%$ des Kontrollschwellenwertes und die Vorimpulse wurden dann in $10 \%$ - Schritten reduziert bis auf den $-100 \%$ hyperpolarisierenden Vorimpuls. Wiederum wurden die Testimpulse mit konditionierenden Reizen und der $1 \mathrm{~ms}$ lange Testimpuls allein abwechselnd gemessen und verglichen. Gemessen wurde die Intensität, die benötigt wurde um den Kontrollschwellenwert zu erreichen (s. Abb. 6 und 8). Die resultierende Kurve liefert Informationen über die einwärts-gleichrichtenden Ströme und ist dementsprechend eine Darstellung der Beziehung zwischen elektrischer Spannung (V) und dem resultierenden lonenstrom (I) (Jankelowitz et al. 2007b, Tomlinson et al. 2010b). Die Kurve im rechten oberen Quadranten zeigt den auswärts gerichteten Strom (= outward rectification), der durch die Aktivierung von langsamen und schnellen $\mathrm{K}^{+}$-Kanäle während der Depolarisation entsteht (Tomlinson et al. 2010b). Die Kurve im linken unteren Quadranten spiegelt hingegen den nach innen gerichteten Strom (= inward rectification) wieder, der durch den $\mathrm{I}_{\mathrm{H}}$-Strom hervorgerufen wird (Krishnan et al. 2009). Je steiler die Kurven sind, desto höher ist die Leitfähigkeit für den jeweiligen Strom (s. Abb. 6) 


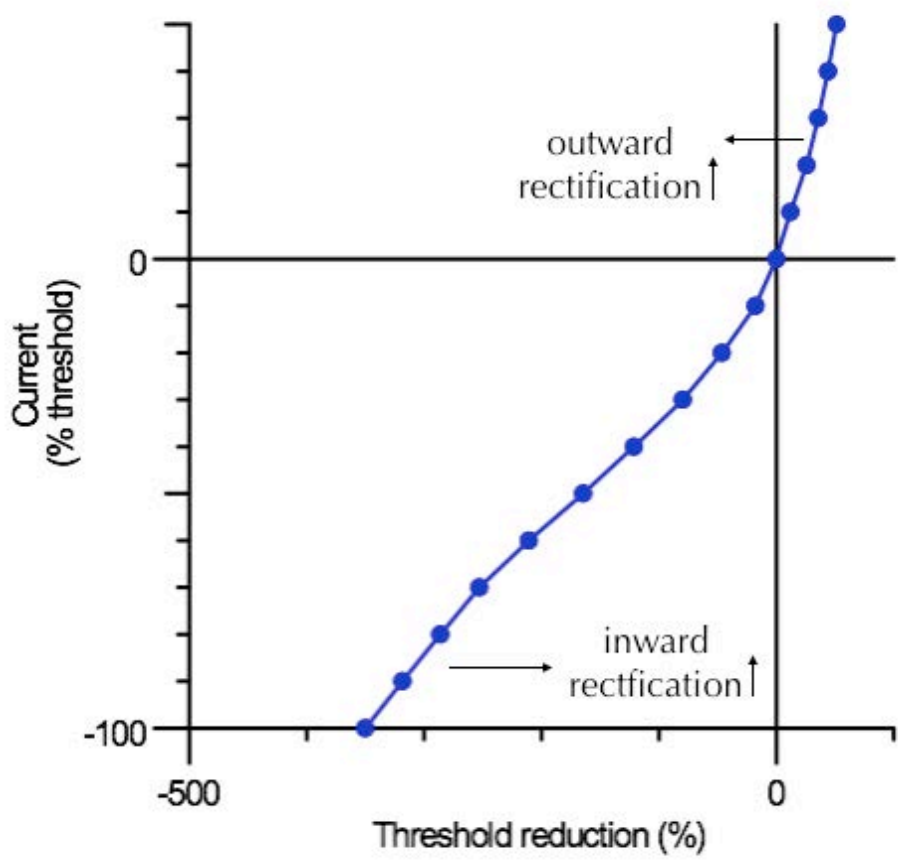

Abbildung 6 Current/threshold relationship. Im rechten oberen Quadranten ist die Antwort auf depolarisierende Reize zu sehen, die das Ausmaß der outward rectification darstellt. Je steiler die Kurve, desto höher ist die outward rectification und damit die Aktivtät der schnellen und langsamen $\mathrm{K}^{+}$Kanäle. Im linken unteren Quadranten ist hingegen die inward rectification abgebildet, die durch hyperpolarsierende konditionierende Reize aktiviert wird. Sie ist ein Ausdruck der Aktivität der HCNKanäle, welche bei einem steileren Verlauf der Kurve gesteigert ist.

\subsubsection{Recovery cycle}

Ein supramaximaler konditionierender Stimulus wurde abgeben und zu 18 unterschiedlichen Zeitpunkten danach (zwischen 2 ms und 200 ms) gemessen, welche Reizintensität benötigt wurde, um den Schwellenwert zu erreichen. Drei Reizkombinationen wurden nacheinander getestet: (i) $1 \mathrm{~ms}$ langer Testimpuls allein (um den Kontrollwert zu erfassen); (ii) $1 \mathrm{~ms}$ langer supramaximaler konditionierender Impuls allein; (iii) konditionierender Vorimpuls + Testimpuls. Bevor die Antwort auf den Testimpuls gemessen wurde, wurde die Antwort auf (ii) von der Antwort auf (iii) abgezogen, da sich die Antworten auf den konditionierenden Reiz und den Testimpuls kurzzeitig überlappen können, insbesondere dann, wenn ein sehr kurzer konditionierender Reiz abgegeben wurde. Aus dem recovery cycle wurden drei Parameter gemessen: die relative Refraktärzeit, definiert als das Intervall zwischen den Reizen, in dem die Reizintensität, die benötigt wird, um den 
Schwellenwert zu erreichen, wieder auf den Kontrollschwellenwert abfällt; die Phase der Übererregbarkeit, in der die Reizintensität geringer ist als bei dem Kontrollimpuls und die Phase der Untererregbarkeit, in der die Reizintensität deutlich über der des Kontrollimpulses liegt (s. Abb. 7). Die Refraktärzeit wird länger, wenn die Membran depolarisiert ist, da die $\mathrm{Na}^{+}-$Kanäle spannungsabhängig sind (Burke et al. 1998, Krishnan et al. 2009). Während des Ruhemembranpotentials sind einige $\mathrm{Na}^{+}-$Kanäle inaktiviert (Burke et al. 2001). Wenn die Membran leicht hyperpolarisiert wird, wird die Inaktivierung aufgehoben und die Refraktärzeit wird kürzer (Burke et al. 2001).

Die Phase der Übererregbarkeit entsteht durch ein depolarisierendes Nachpotential nach der Reizweiterleitung (Barrett und Barrett 1982, Baker et al. 1987, Krishnan et al. 2009). Die letzte Phase, bevor die Membran wieder inr Ruhepotential erreicht, zeichnet sich dadurch aus, dass das Axon schlechter erregbar ist (= subexcitability). Sie entsteht, da langsame $\mathrm{K}^{+}$-Kanäle durch die Depolarisation während der Reizweiterleitung aktiviert werden und während der langsamen Deaktivierung eine Hyperpolarisation erzeugen (Baker et al. 1987, Taylor et al. 1992, Krishnan et al. 2009). Die Messung des recovery cycles eines Probanden ist in Abbildung 8 zu sehen.

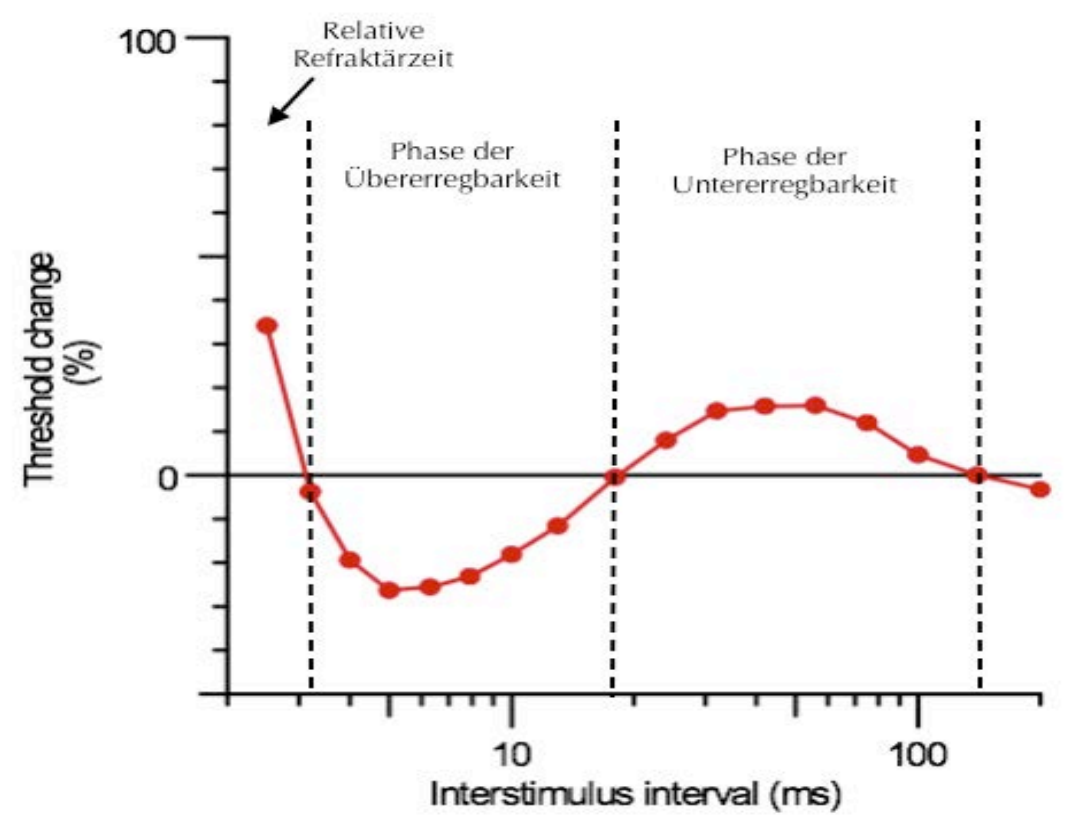

Abbildung 7 Phasen des recovery cycles nach einem supramaximalen Reiz. Die relative Refraktärzeit wird zunächst gefolgt von einer Phase der Übererregbarkeit (superexcitabilty), an die sich dann eine Phase der Untererregbarkeit (subexcitability) anschließt. 


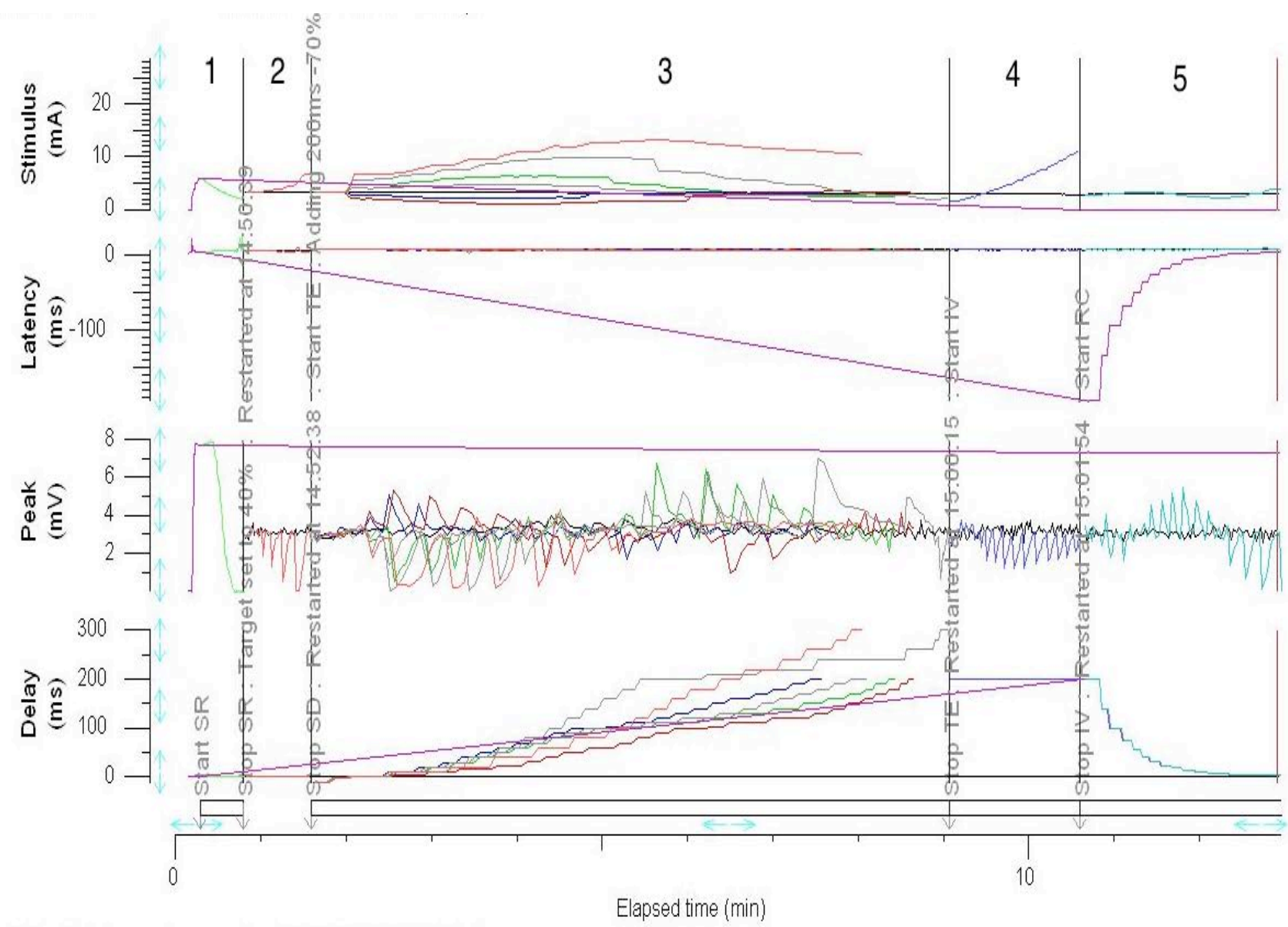

Abbildung 8 Beispiel für die Messung eines Probanden mit 1 = stimulus-response-curve, 2 = strengthduration properties, $3=$ threshold electrotonus, $4=$ current/threshold relationship, $5=$ recovery cycle. Von oben nach unten sind die Amplituden der Impulse (= Stimulus in $\mathrm{mA}$ ), gefolgt von der Latenzzeit (= Latency in ms), der Höhe des Summenaktionspotential als Antwort des Muskels (= Peak in mV) und der Verzögerung zwischen konditionierendem Reiz und Testimpuls aufgetragen.

\subsection{Definitionen der Exzitabilitätsparamter}

Tabelle 1 gibt einen Überblick über die Bedeutung der einzelnen Exzitabilitätsparameter, die mithilfe des threshold trackings bestimmt wurden. 
Tabelle 1 Definitionen der Exzitabilitätsparameter, die verwendet wurden, um die Daten der Patienten mit einem BFCS und der gesunden Kontrollgruppe zu vergleichen.

Exzitabilitätsparameter $\quad$ Definition

\section{Stimulus-response-curve}

Stimulus (mA) für $50 \%$

Intensität des Reizes, der benötigt wird, um 50\% des maximalen Summenaktionspotentials des Muskels auszulösen

Peak response $(\mathrm{mV})$

Amplitude des maximalen Summenaktionspotentials des Muskels

Stimulus-response (slope)

Steigung der stimulus-response-curve

\section{Strength-duration}

Strength-duration time constant Abgeschätzt durch den Schnittpunkt mit der x-Achse der linearen (ms) Regressionslinie (vgl. Abb. 4)

Rheobase $(\mathrm{mA})$

Abgeschätzt durch die Steigung der Kurve in Abb. 4 (vgl. Abb. 3)

\section{Threshold electrotonus}

(die Schwellenwertabnahme wird in Prozent des Kontrollschwellenwertes angeben)

TEd (peak) (20 oder 40)

Maximale Schwellenwertabnahme während des depolarisierenden Stroms (bei 20 oder $40 \%$ des Kontrollimpulses)

$\mathrm{TEd}^{20}$ (von...bis in ms)

Mittlere prozentuale Schwellenwertabnahme zwischen der angegebenen Zeitspanne für den 20\%-depolarisierenden Strom

$\mathrm{TEd}^{40}$ (von...bis in $\mathrm{ms}$ )

$\operatorname{TEd}^{40}$ (undershoot)

Mittlere prozentuale Schwellenwertabnahme zwischen der angegebenen Zeitspanne für den $40 \%$-depolarisierenden Strom

$\operatorname{TEd}^{40}$ (Accommodation)

Minimum der prozentualen Schwellenwertabnahme nach dem $40 \%$ depolarisierenden Strom

S2 accommodation

Höhe des Abfalls vom Maximum der Kurve während der 100 ms langen Depolarisation

Höhe des Abfalls während der gesamten S2-Phase, also von der größten Schwellenwertabnahme bis zum Plateau des 40\%-depolarisierenden Stroms

Accommodation half-time (in $\mathrm{ms}$ )

$\mathrm{TEh}^{20}$ (von....bis in ms)

$\operatorname{TEh}^{40}$ (von...bis in ms)

$\operatorname{TEh}^{40}$ (slope 101-140ms)

$\mathrm{TEh}^{40}$ (overshoot)

TEh (peak) (-70 oder -100\%)

S3 $(-70 \%$ oder $-100 \%)$
Zeit vom Beginn des depolarisierenden Stroms bis die Schwellenwertabnahme die Hälfte zwischen dem Maximum und dem Plateau erreicht hat

Mittlere prozentuale Schwellenwertabnahme zwischen der angegebenen Zeitspanne für den 20\%-hyperpolarisierenden Strom

Mittlere prozentuale Schwellenwertabnahme zwischen der angegebenen Zeitspanne für den 40\%-hyperpolarisierenden Strom

Mittlere Steigung der Kurve zwischen der angegebenen Zeitspanne nach dem 40\%-hyperpolarisierenden Strom

Maximum der prozentualen Schwellenwertabnahme nach dem $40 \%$ hyperpolarisierenden Strom

Maximale Schwellenwertabnahme während des hyperpolarisierenden Stroms (bei -70 oder $-100 \%$ des Kontrollimpulses)

Höhe der S3-Phase (Abnahme des Schwellenwertes nach dem tiefsten Punkt der Kurve nach der Hyperpolarisation von $-70 \%$ oder $-100 \%)$ 


\begin{tabular}{|c|c|}
\hline \multicolumn{2}{|c|}{ Current/threshold relationship (I/V) } \\
\hline Minimum I/V slope & Geringste Steigung der I/V-Kurve (Minimum der Kurve in Abb. 13B) \\
\hline Resting I/V slope & $\begin{array}{l}\text { Berechnet aus den polarisierenden Strömen zwischen }-10 \% \text { und } \\
+10 \% \text { um den Ruheschwellenwert (entspricht dem threshold von } 0 \text { in } \\
\text { Abb. 13B) }\end{array}$ \\
\hline Hyperpolarized I/V slope & $\begin{array}{l}\text { Steigung der I/V-Kurve (Abb. 13A) an ihrem meist hyperpolarisierten } \\
\text { Punkt (in Abb. 13B der Punkt ganz links) }\end{array}$ \\
\hline \multicolumn{2}{|l|}{ Recovery cycle } \\
\hline Superexcitability (\%) & Maximale Schwellenwertabnahme in der Phase der Übererregbarkeit \\
\hline Superexcitability at ...ms & $\begin{array}{l}\text { Maximale Schwellenwertabnahme in der Phase der Übererregbarkeit } \\
\text { zu bestimmten Zeitpunkten }\end{array}$ \\
\hline Subexcitability (\%) & Maximale Schwellenwertzunahme in der Phase der Untererregbarkeit \\
\hline Relative Refraktärzeit (ms) & $\begin{array}{l}\text { Dauer der Phase zwischen dem Zeitpunkt, an dem kein Aktions- } \\
\text { potential auslösbar ist, und dem Beginn der Übererregbarkeit }\end{array}$ \\
\hline Latency (ms) & $\begin{array}{l}\text { Gibt die Dauer vom Beginn des } 1 \mathrm{~ms} \text { langen Testimpulses bis zur } \\
\text { größten Amplitude an und wird über die gesamte Zeit der Messung } \\
\text { gemittelt }\end{array}$ \\
\hline
\end{tabular}

\subsection{Statistische Auswertung}

Die Daten der Patienten, die unter einem BFCS litten, wurden mit den Daten der gesunden Kontrollgruppe mittels eines ungepaarten Student-t-Tests auf signifikante Unterschiede geprüft. Angegebenen wird jeweils der Mittelwert und der Standardfehler, wobei ein p-Wert < 0,05 als signifikant angesehen wurde. Die Ergebnisse des Student-tTests sind in Tabelle 3 dargestellt. Da einige Parameter der elektrischen Erregbarkeit eines Nerven signifikant vom Alter abhängen (Jankelowitz et al. 2007b, Bae et al. 2008) und einige von der Hauttemperatur (Kiernan et al. 2001a), wurden die beiden Gruppen auf ein Alter und eine Hauttemperatur angepasst, die sich nicht signifikant voneinander unterschieden. Die Datenanalyse und die Graphiken wurden aus der Qtrac Software (Version 13/6/2011) exportiert. 


\section{Ergebnisse}

\subsection{Allgemeines}

\subsubsection{Probandencharakteristik}

Die 18 Probanden mit dem BFCS waren zwischen 24 und 71 Jahren alt (Durchschnittsalter 57,4 Jahre) und zeigten, außer spontaner Aktivität in Form von Faszikulationen, keinerlei Pathologien im EMG wie Duplets, Triplets oder Multiplets, normale NLG und DML, sodass insbesondere ein Karpal-Tunnel-Syndrom, eine Polyneuropathie und eine Neuromyotonie ausgeschlossen wurden. Der Antikörpernachweis insbesondere gegen die VGKC war bei allen Patienten negativ. Kein Patient litt an einer Vorerkrankung oder nahm eine Vormedikation ein, die bekannt dafür ist, Muskelkrämpfe zu verursachen.

Tabelle 2 gibt eine Übersicht über die genauen Beschwerden jedes einzelnen gemessenen BFCS-Patienten. Alle BFCS-Patienten klagten über Muskelkrämpfe, die mindestens einmal pro Woche auftraten, wobei 11 sogar täglich von diesen betroffen waren. Die Patienten nahmen keine Medikamente ein, die die Exzitabilitätsmessungen hätten beeinflussen können, wie zum Beispiel Carbamazepin oder Gabapentin. Wenn ein Patient mit einem membranstabilisierenden Medikament oder einem Chinin behandelt wurde, so wurde dieses Medikament mindestens 48 Stunden vor der Messung abgesetzt. Abgesehen von vier Patienten, litten alle unter Muskelkrämpfen in der oberen Extremität, wobei dann immer die Hand betroffen war. Die Stärke des Schmerzes während eines Krampfes wurde von den Patienten anhand einer Schmerzskala von 0 bis 10 angegeben, wobei 0 kein Schmerz wäre und 10 der stärkste Schmerzreiz, den sie sich vorstellen konnten. Der Schmerzreiz lag bei allen Patienten zwischen 3 und 8 (Mittelwert $=5,68$ ). Die Dauer der Krämpfe schwankte zwischen einigen Sekunden und mehreren Minuten, wobei nur ein Patient eine Dauer von mehr als 15 Minuten pro Muskelkrampf angab.

Die 15 gesunden Probanden waren zwischen 32 und 70 Jahre alt (Durchschnittsalter 52,7 Jahre) und nahmen außer oralen Kontrazeptiva und L-Thyroxin keinerlei Medikamente ein. 
Tabelle 2 Informationen zu den Beschwerden der einzelnen BFCS-Patienten

\begin{tabular}{|c|c|c|c|c|c|}
\hline \multirow[b]{2}{*}{ Patient } & \multirow[b]{2}{*}{ Alter } & \multicolumn{4}{|c|}{ Muskelkrämpfe } \\
\hline & & $\begin{array}{l}\text { Lokalisation } \\
\text { (OE= obere Extremität) } \\
\text { (UE= untere Extremität) }\end{array}$ & $\begin{array}{l}\text { Frequenz } \\
\text { (in Tagen pro } \\
\text { Woche) }\end{array}$ & $\begin{array}{c}\text { Dauer } \\
\text { (in min) }\end{array}$ & $\begin{array}{c}\text { Stärke des } \\
\text { Schmerzes } \\
(0-10)^{\star}\end{array}$ \\
\hline 1 & 50 & $\mathrm{OE}+\mathrm{UE}$ & 7 & 10 & $3-4$ \\
\hline 2 & 45 & $\mathrm{OE}+\mathrm{UE}$ & 3 & 0.5 & $5-6$ \\
\hline 3 & 69 & UE & 7 & $5-10$ & 8 \\
\hline 4 & 71 & UE & $1-2$ & $5-10$ & 7 \\
\hline 5 & 69 & $\mathrm{OE}+\mathrm{UE}$ & $2-3$ & $1-2$ & $7-8$ \\
\hline 6 & 46 & $\mathrm{OE}+\mathrm{UE}$ & 7 & 0.5 & 3 \\
\hline 7 & 56 & OE & $2-3$ & k.A. & k.A. \\
\hline 8 & 68 & $\mathrm{OE}+\mathrm{UE}$ & 7 & 3 & 7 \\
\hline 9 & 71 & $\mathrm{OE}+\mathrm{UE}$ & 7 & 0.5 & 6 \\
\hline 10 & 46 & $\mathrm{OE}+\mathrm{UE}$ & 7 & 0.5 & $4-5$ \\
\hline 11 & 58 & $\mathrm{OE}+\mathrm{UE}$ & 7 & $5-6$ & 8 \\
\hline 12 & 70 & $\mathrm{OE}+\mathrm{UE}$ & $2-3$ & 15 & 4 \\
\hline 13 & 51 & $\mathrm{OE}+\mathrm{UE}$ & 7 & k.A. & k.A. \\
\hline 14 & 53 & $\mathrm{OE}+\mathrm{UE}$ & 7 & k.A. & k.A. \\
\hline 15 & 24 & UE & 1 & $60-120$ & $6-7$ \\
\hline 16 & 53 & UE & 5 & $10-15$ & $4-5$ \\
\hline 17 & 69 & $\mathrm{OE}+\mathrm{UE}$ & 7 & k.A. & k.A. \\
\hline 18 & 64 & $\mathrm{OE}+\mathrm{UE}$ & 7 & 0.5 & $4-5$ \\
\hline
\end{tabular}




\subsubsection{Allgemeine Daten der Messung}

Die Exzitabilitätsmessung mittels des Trond-Protokolls dauerte bei den Patienten mit einem BFCS im Mittel 12,58 min (10,73 - 16,68 min) und bei der Kontrollgruppe 14,51 min (12,37 - 17,96 min). Die Hauttemperatur, die im Bereich der Stimulationselektrode über dem N. medianus gemessen wurde, lag im Mittel bei $32,27^{\circ} \mathrm{C}$ für die BFCS-Patienten und bei $32,75^{\circ} \mathrm{C}$ für die gesunde Kontrollgruppe.

Für alle Probanden war die Messung zwar etwas unangenehm, aber insgesamt gut tolerierbar.

\subsection{Exzitabilitätsmessung}

Die Ergebnisse der Messung der Patienten und der Kontrollgruppe sind in Tabelle 3 zur Übersicht vergleichend dargestellt. Im Material und Methodenteil wurde bereits die Bedeutung der einzelnen Exzitabilitäsparamter näher erläutert (s. Tabelle 1). Die stärksten Unterschiede zeigten sich im threshold electrotonus, der current/threshold relationship und der stimulus-response-curve (s. Abb. 9, 10, 11, 12 und 13). 
Tabelle 3 Vergleich der Exzitabilitätsparameter des N. medianus zwischen Patienten mit einem BFCS und einer gesunden Kontrollgruppe

\begin{tabular}{ccccc}
\hline Exzitabilitätsparameter & Kontrollgruppe & BFCS-Patienten & $t$ (df) & $p$ \\
& $(M W+/-S E ; n=15)$ & $(M W+/-S E ; n=18)$ & $(d f=31)$ & \\
\hline
\end{tabular}

\section{Stimulus-reponse-curve}

Stimulus (mA) für $50 \%$

$4.758 x \mid / 1.07$

$3.897 x \mid / 1.07$

2.018

$0.04976^{*}$

Peak response $(\mathrm{mV})$

$7.362 x / 1.09$

$6.233 x \mid / 1.11$

1.241

0.22184

Stimulus-response (slope)

$5.014 x \mid / 1.08$

$4.947 x \mid / 1.07$

$135 \quad 0.8631$

\section{Strength-duration}

Strength-duration time constant (ms)

Rheobase (mA)

$\begin{array}{ccrr}0.5194+\mid-0.0334 & 0.4549+\mid-0.0165 & 1.825 & 0.07437 \\ 3.088 x \mid / 1.09 & 2.606 x \mid / 1.07 & 1.53 & 0.13243\end{array}$

\section{Threshold electrotonus}

$\mathrm{TEd}^{20}$ (10-20ms) (\%)

$\mathrm{TEd}^{20}$ (peak) (\%)

$\begin{array}{cccl}35.71+\mid-0.66 & 34.72+\mid-0.492 & 1.227 & 0.22724 \\ 38.29+\mid-0.781 & 37.07+\mid-0.501 & 1.357 & 0.18159 \\ 68.43+\mid-0.77 & 66.43+\mid-0.68 & 1.949 & 0.0575 \\ 51.83+\mid-1.01 & 50.71+\mid-0.995 & 0.78 & 0.44675 \\ 68.35+\mid-0.704 & 66.22+\mid-0.693 & 2.145 & \mathbf{0 . 0 3 7 9 2} \\ -21.44+\mid-1.13 & -18.82+\mid-0.418 & 2.322 & \mathbf{0 . 0 2 5 6 6 ^ { \star }} \\ 43.98+\mid-0.718 & 43.22+\mid-0.677 & 0.776 & 0.44936 \\ 24.29+\mid-0.935 & 22.77+\mid-0.62 & 1.396 & 0.16932 \\ 24.37+\mid-0.934 & 23+\mid-0.604 & 1.266 & 0.21253 \\ 41.52+\mid-1.34 & 41.39+\mid-1.33 & 0.071 & 0.90103 \\ -37.89+\mid-0.97 & -34.85+\mid-0.554 & 2.832 & \mathbf{0 . 0 0 7 8 7 ^ { \star \star }} \\ -76.46+\mid-1.65 & -71.65+\mid-0.92 & 2.657 & \mathbf{0 . 0 1 1 9 1} \\ -95.41+\mid-2.39 & -87.5+\mid-1.46 & 2.925 & \mathbf{0 . 0 0 6 3} \\ -123.6+\mid-3.92 & -107.1+\mid-3.37 & 3.206 & \mathbf{0 . 0 0 3 1 8 ^ { \star \star }} \\ 2.334+\mid-0.109 & 1.903+\mid-0.0729 & 3.374 & \mathbf{0 . 0 0 2 1 ^ { \star \star }} \\ 19.84+\mid-1.68 & 15.76+\mid-0.69 & 2.397 & \mathbf{0 . 0 2 1 6 5 ^ { \star }} \\ -265+\mid-8.32 & -227.7+\mid-6.99 & 3.462 & \mathbf{0 . 0 0 1 6 8 ^ { \star * }} \\ 19.43+\mid-4.24 & 17.75+\mid-2.07 & 0.376 & 0.70902 \\ -387.1+\mid-11.4 & -342+\mid-9.54 & 3.06 & \mathbf{0 . 0 0 4 5 4 ^ { \star * }} \\ 79.87+\mid-4.85 & 64.22+\mid-4.18 & 2.461 & \mathbf{0 . 0 1 8 7 5 ^ { \star }}\end{array}$

$\operatorname{TEd}^{40}$ (10-20ms) (\%)

$\mathrm{TEd}^{40}(40-60 \mathrm{~ms})(\%)$

$\operatorname{TEd}^{40}$ (peak) (\%)

$\mathrm{TEd}^{40}$ (undershoot) (\%)

$\mathrm{TEd}^{40}(90-100 \mathrm{~ms})(\%)$

$\mathrm{TEd}^{40}$ (Accommodation) (\%)

S2 accommodation (\%)

Accommodation half-time (ms)

$\operatorname{TEh}^{20}(10-20 \mathrm{~ms})(\%)$

$\mathrm{TEh}^{40}(10-20 \mathrm{~ms})(\%)$

$\mathrm{TEh}^{40}(20-40 \mathrm{~ms})(\%)$

$\mathrm{TEh}^{40}(90-100 \mathrm{~ms})(\%)$

$\mathrm{TEh}^{40}$ (slope 101-140ms)

TEh $^{40}$ (overshoot) (\%)

TEh (peak,-70\%) (\%)

S3 $(-70 \%)(\%)$

TEh (peak,-100\%) (\%)

S3 (-100\%) (\%) 


\section{Current/threshold relationship (I/V)}

Minimum I/V slope

$0.2456+\mid-0.0103$

$0.2899+\mid-0.0126$

$2.644 \mathbf{0 . 0 1 2 2 6}{ }^{\star}$

Resting I/V slope

$0.6822+\mid-0.0364$

$0.6503+\mid-0.0255$

$0.736 \quad 0.47361$

Hyperpolarized I/V slope

$0.4061+\mid-0.0225$

$0.3814+\mid-0.0101$

$1.057 \quad 0.2991$

\section{Recovery cycle}

Superexcitability (\%)

Superexcitability at $5 \mathrm{~ms}$

$-22.37+\mid-1.41$

$-22.56+\mid-1.27$

0.105

0.88113

$-22.15+\mid-1.49$

$-22.55+\mid-1.41$

$0.194 \quad 0.82702$

Superexcitability at $7 \mathrm{~ms}$

$-21.23+\mid-1.43$

$-20.4+\mid-1.28$

$0.43 \quad 0.67296$

Subexcitability (\%)

$16.33+\mid-1.07$

$14.54+\mid-0.833$

1.3390 .18725

Relative Refraktärzeit (ms)

$3.157 x \mid / 1.04$

$3.004 x \mid / 1.04$

0.925

0.36532

Refractoriness at $2 \mathrm{~ms}$

$86.77+\mid-11$

$66.56+\mid-12.7$

$1.17 \quad 0.25179$

Latency (ms)

$7.014+\mid-0.223$

$6.808+\mid-0.175$

$0.734 \quad 0.47454$

\section{Probanden}

Alter (Jahre)

$\begin{array}{cccc}52.67+\mid-2.76 & 57.39+\mid-3 & 1.14 & 0.26236 \\ 1.333+\mid-0.126 & 1.722+\mid-0.109 & 2.35 & 0.02407^{*} \\ 32.75+\mid-0.0925 & 32.27+\mid-0.248 & 1.738 & 0.08918\end{array}$

Geschlecht (M=1, $\mathrm{F}=2)$

$32.75+\mid-0.0925$

$32.27+\mid-0.248$

$\begin{array}{ll}1.738 & 0.08918\end{array}$

${ }^{\star} p<0,05,{ }^{* *} p<0,01 ; T E=$ threshold electrotonus, $d=$ depolarisierend, $h=$ hyperpolarisierend, Zahl:

Stärke des konditionierenden Reizes in Prozent des Kontrollimpulses (z.B. $40=40 \%$ des Kontrollschwellenwertes); $t$ und Anzahl der Freiheitsgrade ( $d f=$ degrees of freedom) des Student-t-Tests, $M W=$ Mittelwert, SE = Standardfehler (engl. standard error)

\subsubsection{Stimulus-response-curve}

Die Abbildung 9A zeigt die absoluten Werte der stimulus-response-curve beider Gruppen, die bei einem Testimpuls mit einer Dauer von 1 ms gemessen und dann doppelt logarithmisch aufgetragen wurden. Die graue Kurve stellt dabei die Ergebnisse der Kontrollgruppe und die schwarze die der BFCS-Patienten dar. Die maximale Höhe des Summenaktionspotentials des Muskels zeigte bei den BFCS-Patienten mit 6,23 $\times 1 / 1,11$ $\mathrm{mA}$ keinen signifikanten Unterschied zu der Kontrollgruppe mit 7,36 $\mathrm{x} / / 1,09 \mathrm{~mA}(\mathrm{p}=0,22)$ (s. Abb 6A). Allerdings reichte bei den Patienten mit einem BFCS eine um 18\% geringe Reizintensität aus, um 50\% des maximalen Summenaktionspotentials auszulösen (BFCS $=3.897 \mathrm{x} / 1.07 \mathrm{~mA}, \mathrm{NC}=4.758 \mathrm{x} / 1.07 \mathrm{~mA}, \mathrm{p}=0.04976$ ) (s. Abb. 9A). Somit nahm die Schwelle zur Auslösung eines Aktionspotentials ab, was durch die Verlagerung der Kurve nach links deutlich wird (s. Abb. 9A). 
Die Steigung lässt sich aus der normalisierten stimulus-response-relationship in Abbildung 9B berechnen, in dem die maximale Antwort auf einen 25\% starken Reiz von der maximalen Antwort auf einen 75\% starken abgezogen und das Ergebnis durch die Antwort auf einen 50\% starken Reiz geteilt wird (die Prozentangaben beziehen sich jeweils auf das maximal auslösbare Summenaktionspotential). Diese Kurve wurde auf einer linearen Achse aufgetragen. Die Steigung der beiden Kurven unterschieden sich in den untersuchten Gruppen nicht signifikant voneinander $(B F C S=4.947 \times \mid / 1.07, \quad N C=$ $5.014 \mathrm{x} / 1.08, \quad \mathrm{p}=0.8631$ ) (s. Abb. 9B). Somit war die stimulus-response-curve bei den BFCS-Patienten in ihrer Form nicht verändert.

A

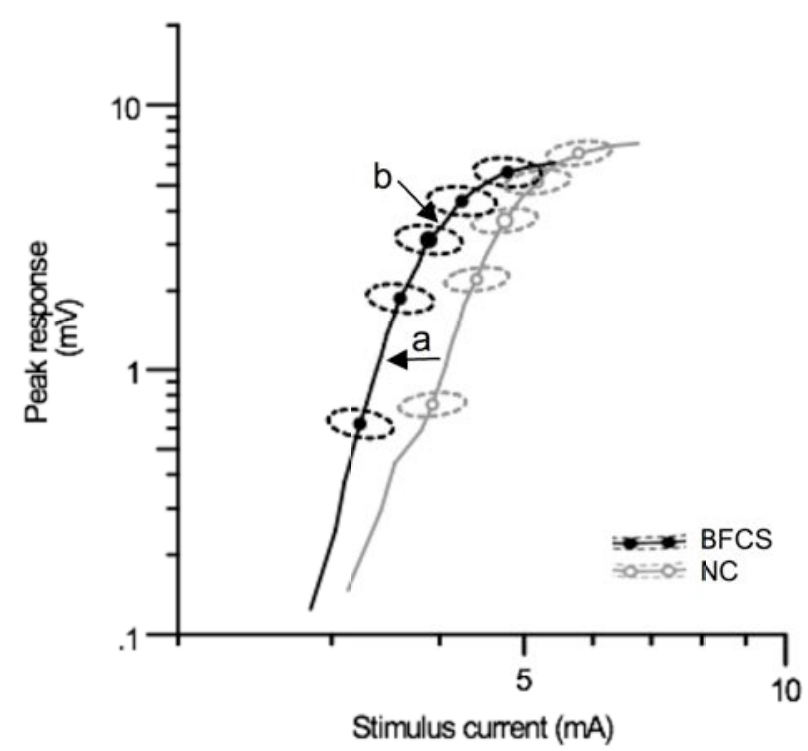

B

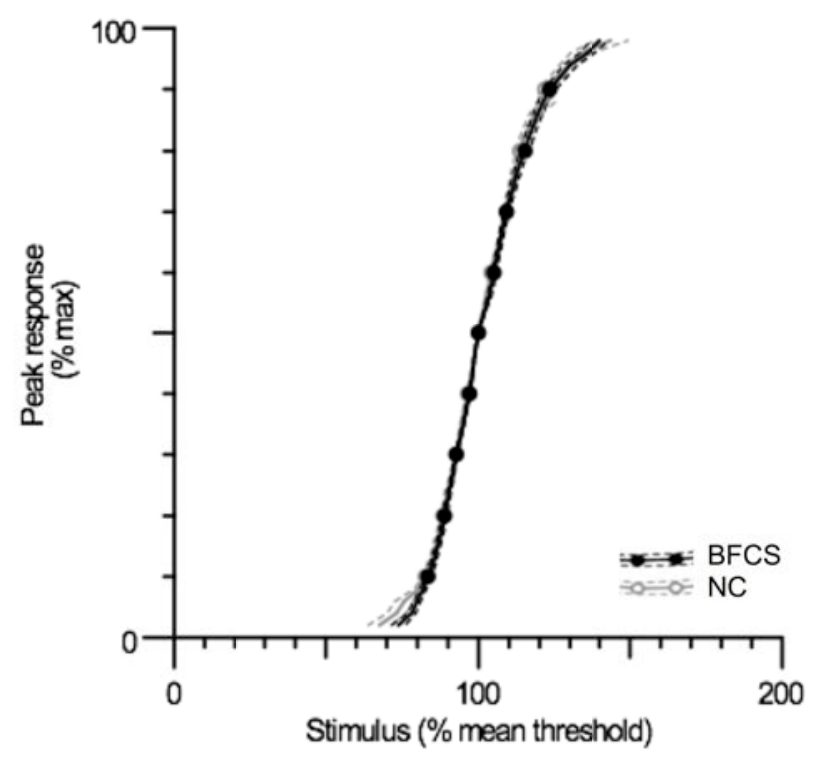

Abbildung 9 Die schwarzen, ausgefültten Kreise zeigen die Daten der Probanden mit einem BFCS und die grauen, offenen die der gesunden Kontrollgruppe, wobei die durchgezogene Linie die Mittelwerte und die gestrichelte den Standardfehler darstellt. (A) Die absoluten Werte der stimulusresponse-curve zeigten, dass die BFCS-Probanden gerade bei niedrigen Intensitäten einen schwächeren Reiz benötigten um die gleiche maximale Antwort wie die Kontrollgruppe zu erzeugen, was durch die Linksverschiebung der Kurve deutlich wird (a). b markiert die Stelle des halbmaximalen Summenaktionspotentials. (B) Die normalisierte stimulus-response-relationship gibt die Daten so wieder, dass der benötigte Reiz zur Auslösung von 50\% des maximalen Summenaktionspotentials einer Intensität von 100\% entspricht und spiegelt die Form der Kurve wider, die bei den BFCSPatienten keine Auffälligkeiten zeigte. 


\subsubsection{Threshold electrotonus}

Der threshold electrotonus gibt Informationen über die nodalen und internodalen Erregbarkeitseigenschaften von Axonen. Dabei wird die Antwort auf depolarisierende konditionierende Reize nach oben und auf hyperpolarisierende konditionierende nach unten aufgetragen, jeweils als Abnahme des Schwellenwertes verglichen mit dem Kontrollschwellenwert (= threshold reduction) (Abb. 10, 11 und 12).

In Abbildung 10A ist der gesamte Elektrotonus dargestellt mit den Veränderungen der Erregbarkeit nach unterschwelligen konditionierenden Reizen von $+40 \%,+20 \%,-20 \%$, $-40 \%,-70 \%$ und $-100 \%$ (von oben nach unten) des unkonditionierten Reizes, der nötig ist um den Kontrollschwellenwert auszulösen. Die Patienten mit einem BFCS zeigten eine geringere Abnahme des Schwellenwertes nach depolarisierenden konditionierenden Reizen und eine geringere Zunahme nach hyperpolarisierenden verglichen mit der Kontrollgruppe.

Die maximale Schwellenwertabnahme ( $\mathrm{TEd}^{40}$ peak) nach einer Reizintensität von $40 \%$ des Kontrollschwellenwertes war 3\% kleiner bei Patienten mit einem BFCS verglichen zu der Kontrollgruppe (BFCS= $66.22+|-0.693 \%, N C=68.35+|-0.704 \%, p=0.03792 ; \mathrm{s}$. Abb. 10B) (Die Werte sind als Reduzierung des Schwellenwertes nach den konditionierenden Reizen verglichen zu dem Schwellenwert nach einem $1 \mathrm{~ms}$ langen Testimpuls in Prozent angegeben). Außerdem zeigte sich eine ähnliche Tendenz auch zwischen 10 und $20 \mathrm{~ms}$ nach einem $40 \%$ depolarisierenden Reiz, da hier die Schwellenwertabnahme bei den BFCS-Patienten ebenfalls $3 \%$ geringer war bei einem fast signifikanten $p$-Wert von 0,0575 (BFCS $=66.43+|-0.68, N C=68.43+|-0.77)$. Des Weiteren fällt hier auf, dass die maximale Schwellenwertzunahme am Ende des depolarisierenden Stroms ( $=\mathrm{TEd}^{40}$ undershoot) in der BFCS-Gruppe 12\% geringer war, verglichen mit der Kontrollgruppe (BFCS $=-18.82+\mid-$ $0.418 \%, N C=-21.44+\mid-1.13 \%, p=0.02566)$ (s. Abb. 10B). 
A

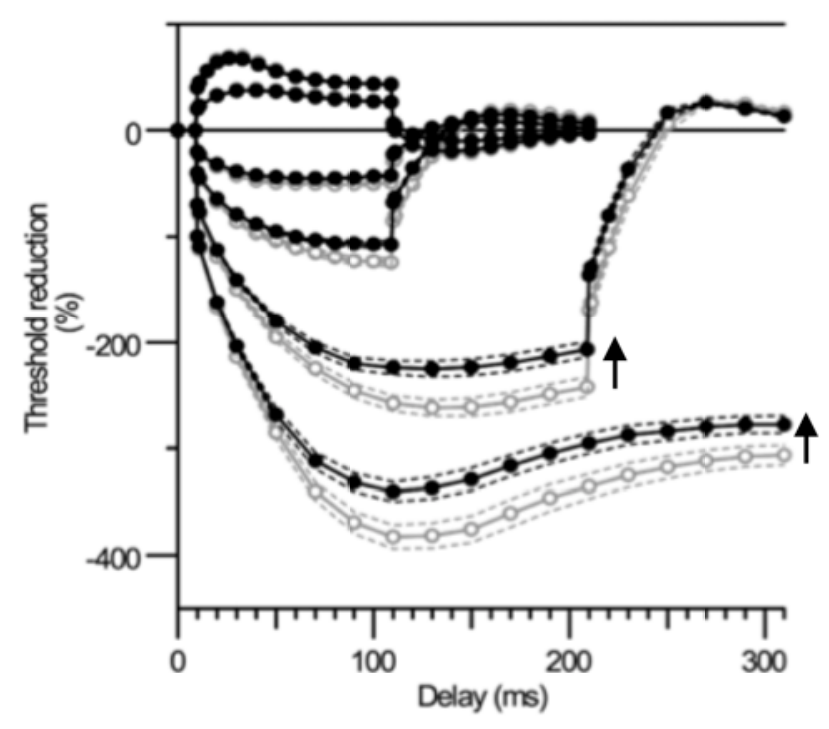

B

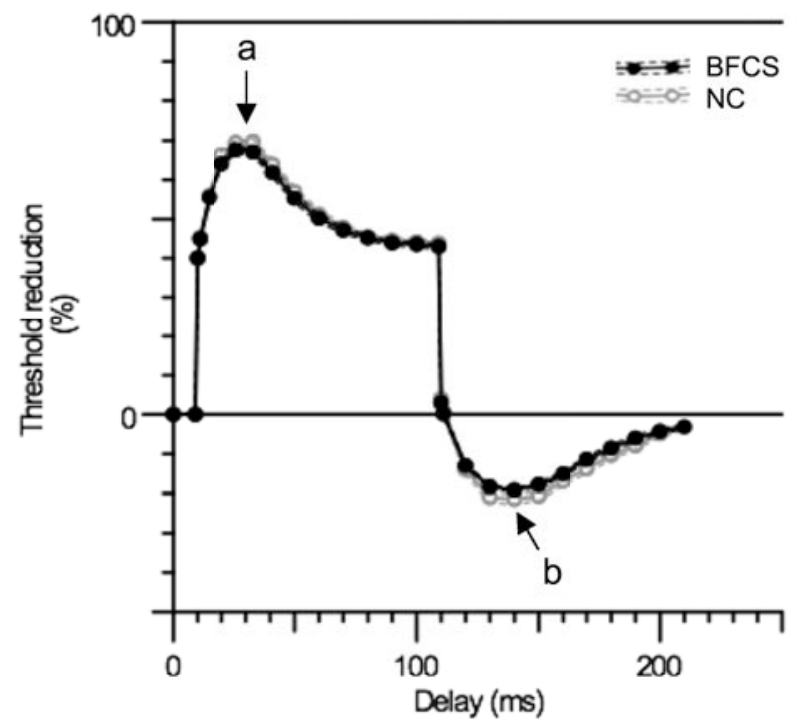

Abbildung 10 Veränderungen im threshold electrotonus bei BFCS-Patienten (schwarz) verglichen mit der gesunden Kontrollgruppe (grau) (Mittelwert und Standardfehler) (A) Abbildung des gesamten threshold electrotonus, wobei die Veränderungen des Schwellenwertes als Antwort auf depolarisierende ( +20 und $+40 \%$ des Kontrollimpulses) konditionierende Reize nach oben und auf hyperpolarisierende (-20,-40,-70 und -100\% des Kontrollimpulses) nach unten aufgetragen wurden. Es zeigte sich vor allem eine geringere Zunahme des Schwellenwertes nach hyperpolarisierenden konditionierenden Reizen. (B) Antwort auf den $+40 \%$ depolarisierenden Reiz. Die BFCS-Patienten zeigten einen niedrigeren Höchstwert (a) und einen kleineren undershoot (b) als die Kontrollgruppe.

Nach dem hyperpolarisierendem konditionierendem Vorimpuls von -20\% des Kontrollschwellenwertes zeigten sich die deutlichsten Unterschiede zwischen 10 und 20 ms nach dem Reiz. Hier hatten die BFCS-Patienten einen $8 \%$ geringeren Anstieg des Schwellenwertes verglichen mit der Kontrollgruppe (BFCS $=-34.85+\mid-0.554 \%, N C=$ $-37.89+\mid-0.97 \%, p=0.00787$ ) (s. Abb. 11A).

Ähnlich sah es auch nach dem 100 ms langen -40\%-hyperpolarisierendem Vorimpuls aus, wie in Abbildung 11B zu sehen ist. Die Patienten mit einem BFCS zeigten einen 6\% niedrigeren Schwellenwert zwischen 10 und 20 ms (BFCS= $-71.65+\mid-0.92 \%, N C=$ $-76.46+\mid-1.65 \%, p=0.01191$ ), 8\% niedriger zwischen 20 und 40 ms (BFCS $=-87.5+\mid-1.46$ $\%, \mathrm{NC}=-95.41+\mid-2.39 \%, \mathrm{p}=0.0063)$ und $13 \%$ niedriger zwischen 90 und $100 \mathrm{~ms}$ nach dem Reiz (BFCS $=-107.1+|-3.37 \%, N C=-123.6+|-3.92 \%, p=0.00318$; siehe auch Abb. 
16A). Die Steigung der Kurve der BFCS-Gruppe war 18\% niedriger zwischen 101 und 140 ms nach dem Reiz verglichen mit der Kontrollgruppe (BFCS $=1.903+\mid-0.0729, \mathrm{NC}=$ $2.334+\mid-0.109, p=0.0021)$. Des Weiteren zeigte sich eine $20 \%$ geringere maximale Schwellenwertabnahme am Ende des hyperpolarisiereden Stroms ( $=\mathrm{TEh}^{40}$ overshoot) bei den BFCS-Probanden (BFCS 15.76+|-0.69 \%, NC= 19.84+|-1.68 \%, $\mathrm{p}=0.02165$ ) (s. Abb. 11B, Sternchen).

A

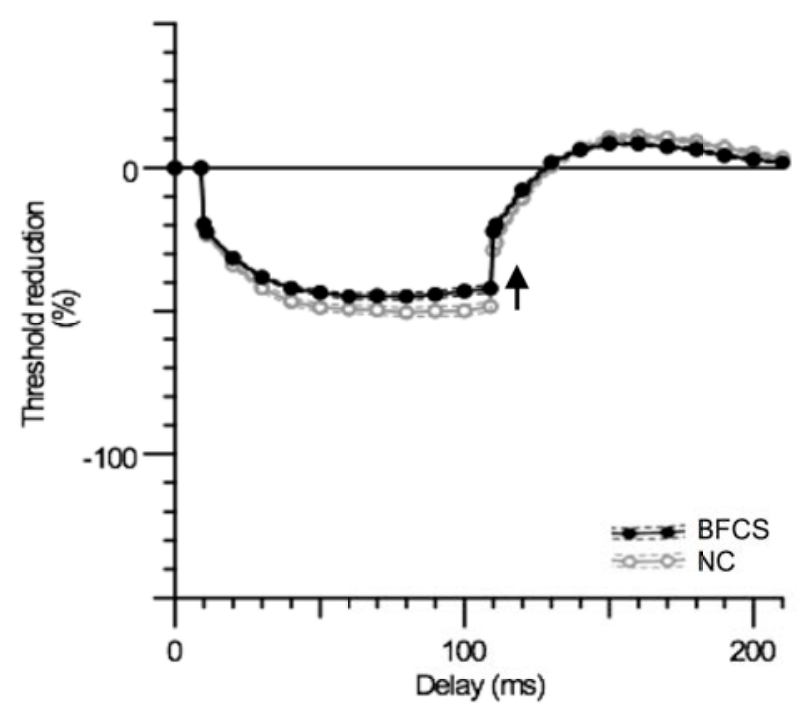

B

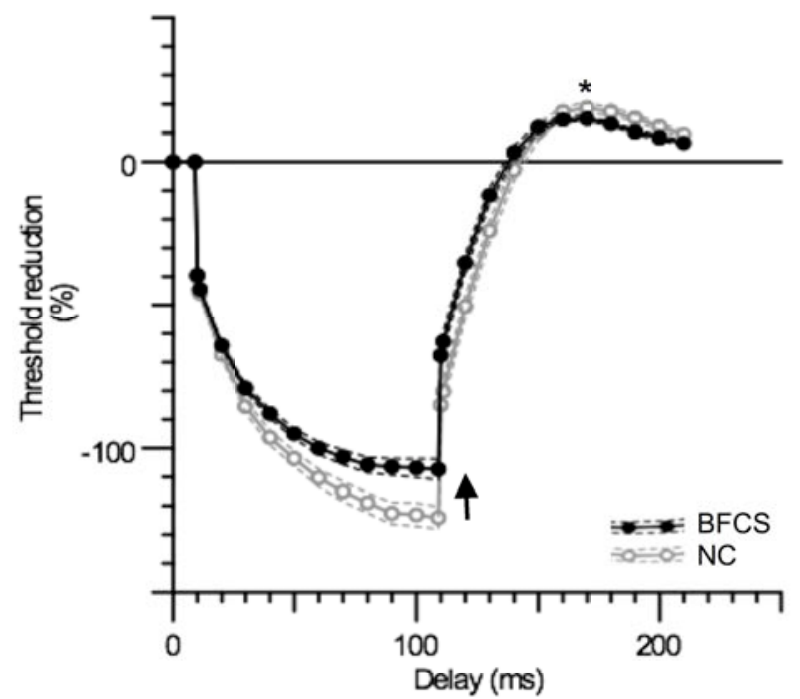

Abbildung 11 Schwarz = BFCS, grau = Kontrollgruppe (Mittelwert und Standardabweichung) (A) Geringere Schwellenwertzunahme nach hyperpolarisierenden konditionierenden Reizen mit -20\% und (B) $-40 \%$ des Kontrollimpulses, was durch die Verlagerung der Kurve nach oben zu weniger negativen Werten deutlich wird. Niedrigerer overshoot bei den BFCS-Patienten (*).

In der Abbildung 12 sind die Ergebnisse der Messung nach 200ms langen konditionierenden Reizen einer Stärke von $-70 \%$ und 300 ms langen mit einer Stärke von -100\% des Kontrollimpulses aufgetragen. Es zeigte sich eine 14\% geringere maximale Zunahme des Schwellenwertes bei den BFCS-Patienten nach -70\% (BFCS $=-227.7+\mid-6.99 \%$, NC= $-265+\mid-8.32 \%, p=0.00168$ ) und $12 \%$ nach $-100 \%$ (BFCS $=-342+|-9.54 \%, N C=-387.1+|-$ $11.4 \%, \quad p=0.00454)$ verglichen mit der Kontrollgruppe. In der Abbildung 16B und C wurden diese Ergebnisse noch einmal zur Übersicht in einem Punktdiagramm dargestellt. 
Die S3-Phase, also die Abnahme des Schwellenwertes nach dem tiefsten Punkt der Kurve nach der Hyperpolarisation, war nach einem -100\% konditionierenden Reiz bei den BFCSPatienten $20 \%$ geringer als bei der Kontrollgruppe (BFCS $=64.22+|-4.18 \%, \mathrm{NC}=79.87+|-$ $4.85 \%, p=0.01875)$.

A

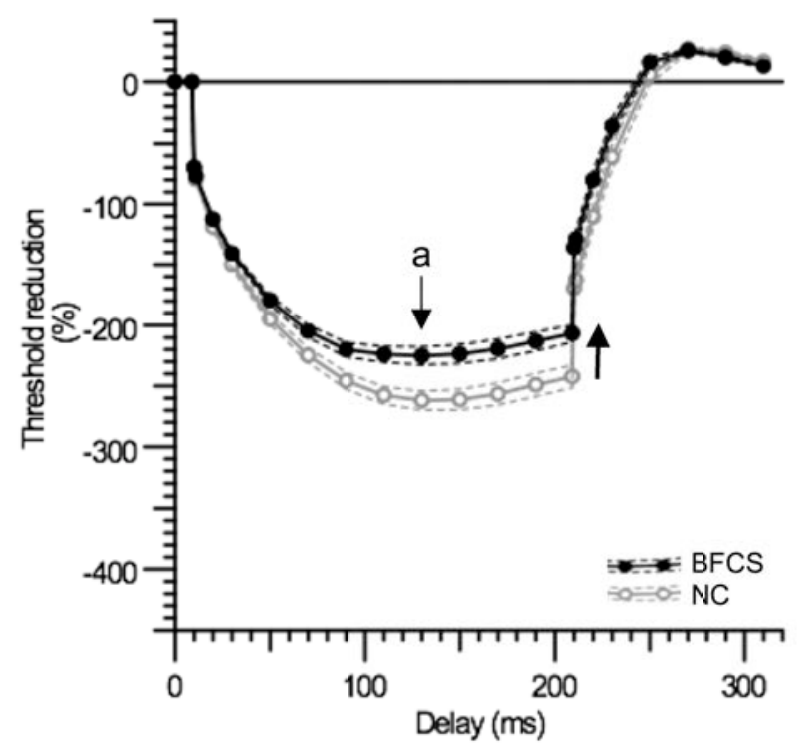

B

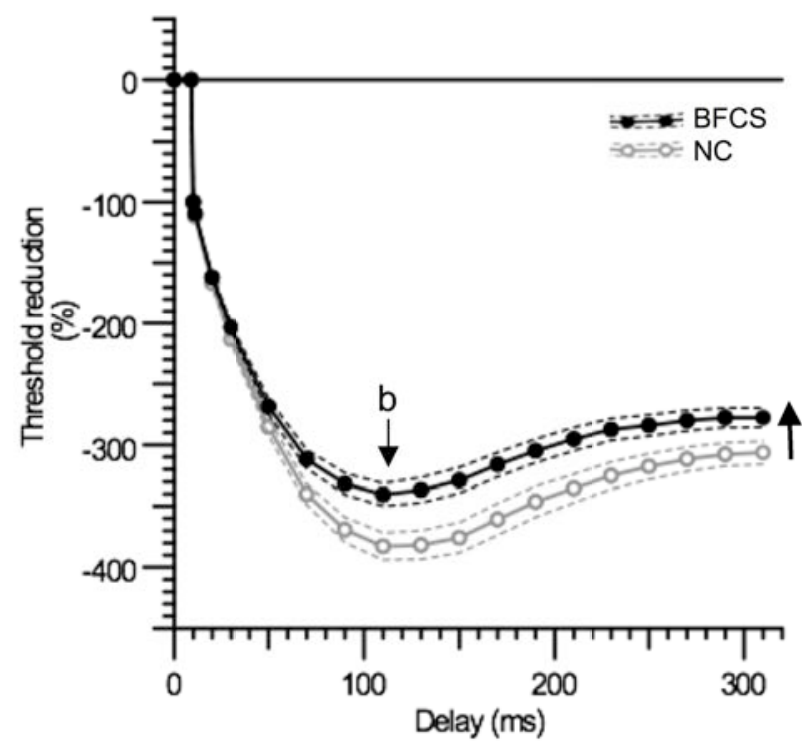

Abbildung 12 Verminderte Schwellenwertzunahme als Antwort auf hyperpolarisierende konditionierende Reize. Schwarz dargestellt sind der Mittelwert und der Standardfehler der Patienten mit einem BFCS und grau die Werte der Kontrollgruppe. (A) Geringere Zunahme des Schwellenwertes als Antwort auf $-70 \%$ und (B) auf -100\% des Kontrollimpulses (Pfeile). Die kleinen Buchstaben markieren die maximale Antwort auf -70\% (TEh (peak, -70\%) = a) in der Abb. 8A und auf -100\% (TEh (peak, $-100 \%)=b)$ in der Abb. 8 B der BFCS-Patienten.

\subsubsection{Current/threshold relationship}

Die Beziehung zwischen dem lonenstrom und der Schwellenwertabnahme wird in den Veränderungen nach 200 ms langen Reizen, die von $50 \%$ bis $-100 \%$ des Impulses reichen, der benötigt wird um den Kontrollschwellenwert zu erzeugen, widergespiegelt.

Die Patienten mit einem BFCS zeigten eine steilere Kurve in hyperpolarisierender Richtung verglichen mit der Kontrollgruppe (s. Abb. 13). In Abbildung 13A wird dies daran deutlich, dass die Kurve der BFCS-Gruppe im linken unteren Quadranten etwas nach rechts verschoben ist. Die Steigung dieser Kurve ist in Abbildung 13B zu sehen. Auf der 
rechten Seite ist die outward und auf der linken die inward rectification zu sehen. Je größer die Steigung ist, desto größer ist die Leitfähigkeit für den jeweiligen Strom. Die kleinste Steigung war dabei um 18\% größer als bei der gesunden Kontrollgruppe (BFCS= $0.2899+|-0.0126, \mathrm{NC}=0.2456+|-0.0103, \mathrm{p}=0.01226$ ) (s. Abb. 13B, a). Abbildung 16D stellt dieses Ergebnis noch einmal übersichtlicher in einem Punktdiagramm da.

A

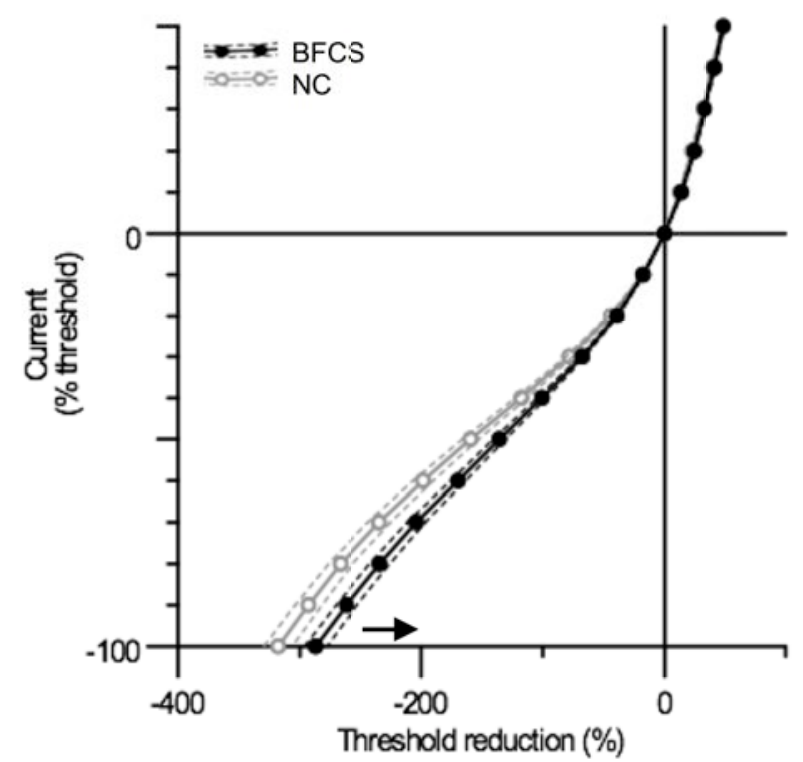

B

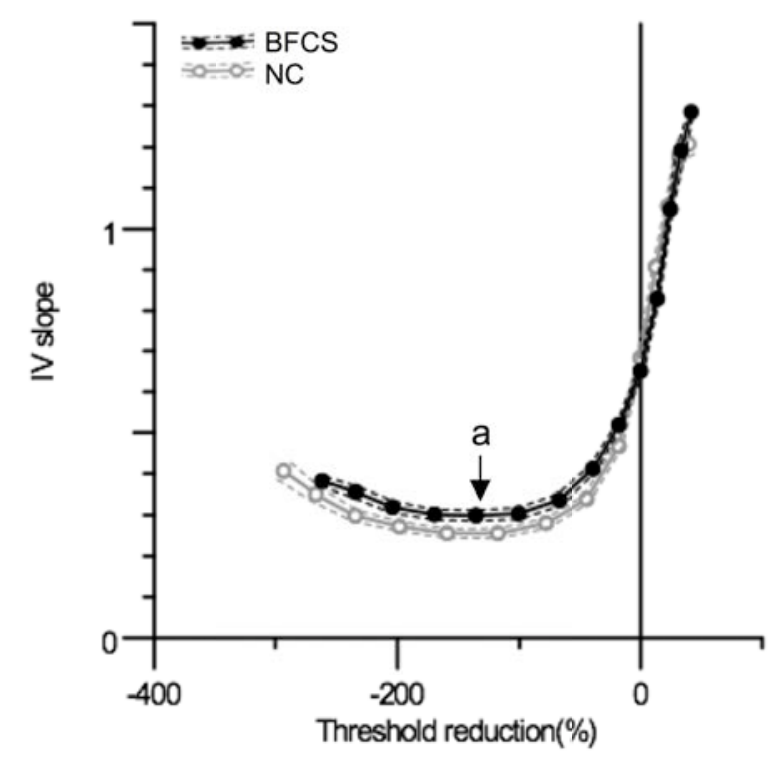

Abbildung 13 Current/threshold-relationship (I/V) (A) Aufgetragen sind die Veränderungen des Schwellenwertes nach 200 ms langen polarisierenden Vorimpulsen als prozentuale Ab- bzw. Zunahme im Vergleich zum Kontrollschwellenwert (= threshold reduction) auf der $x$-Achse gegen den polarisierenden Strom von $+50 \%$ bis $-100 \%$ des Kontrollimpulses auf der $y$-Achse. Die Kurve in der hyperpolarisierenden Richtung (unten links) war steiler für BFCS-Patienten, was durch die Verschiebung nach rechts deutlich wird (Pfeil) (B) Abgebildet ist die Steigung der Kurve aus (A). Deutlich zu sehen ist die größere Steigung für Patienten mit einem BFCS in hyperpolarisierender Richtung. Der Buchstabe a markiert das Minimum der Steigung, welches ebenfalls höher liegt. (schwarze, ausgefüllte Kreise = Patienten mit einem BFCS; graue, offene Kreise = Kontrollgruppe; Mittelwert und Standardfehler (gestrichelte Linie)). 


\subsubsection{Strength-duration relationship}

In der Abbildung 14A sind die Daten der strenght-duration relationship zu sehen als Beziehung zwischen elektrischer Ladung und der Dauer des Reizes. Der threshold charge steht dabei für die Energie des Reizes. Der strength-duration time constant ergibt sich aus dem negativen Schnittpunkt der x-Achse der Regressionslinie. Die Rheobase kann aus der Steigung der Regressionslinie berechnet werden.

Der strength-duration time constant mit 0.4549+|-0.0165 ms für die BFCS-Patienten und $0.5194+\mid-0.0334 \mathrm{~ms}$ für die Kontrollgruppe $(p=0.07437)$, sowie die Rheobase mit 2.606x|/1.07 mA bei den Patienten mit einem BFCS und 3.088x/1.09 mA bei der Kontrollgruppe $(p=0.13243)$ unterschieden sich zwischen den beiden Gruppen nicht signifikant (s. Abb 14A).

A

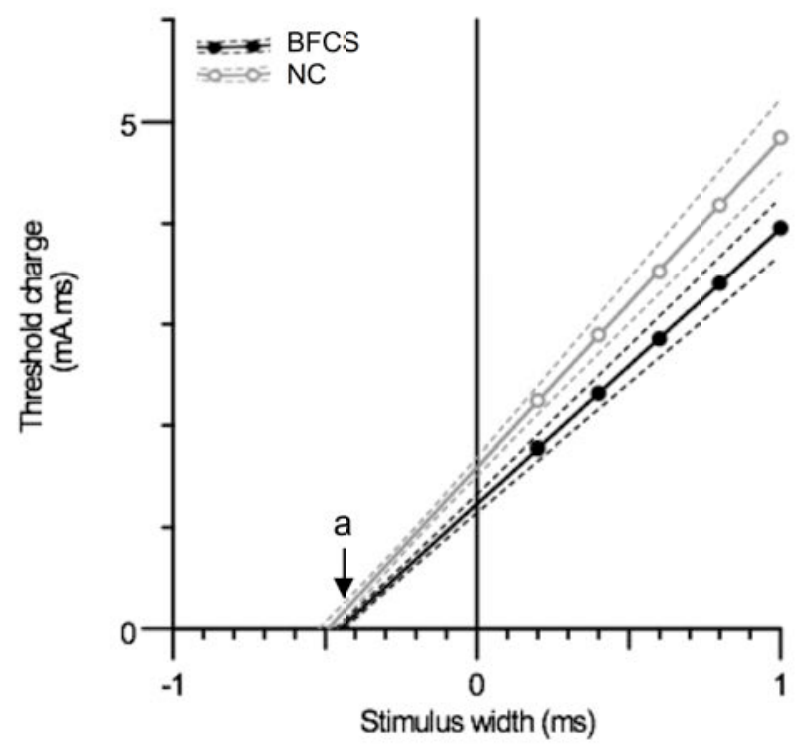

B

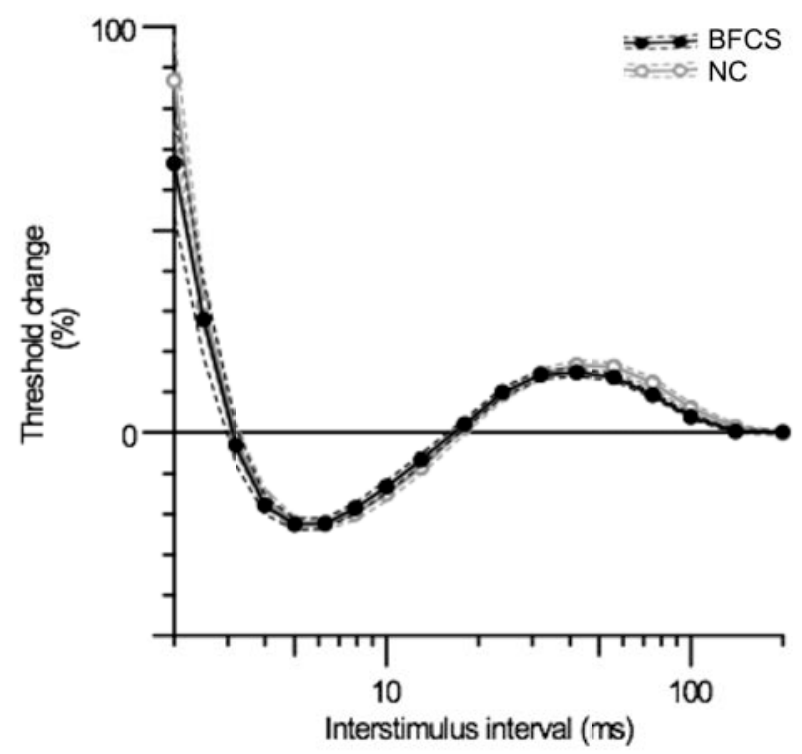

Abbildung 14 Schwarz zeigt die Daten der Probanden mit einem BFCS und grau die der gesunden Kontrollgruppe, wobei die durchgezogene Linie die Mittelwerte und die gestrichelte den Standardfehler darstellt. (A) Aufgetragen ist threshold charge (entspricht der Stromstärke des Schwellenwertes multipliziert mit der Reizdauer) (nach dem Weiss'schen Gesetz) gegen die Reizdauer. Der Schnittpunkt mit der $x$-Achse der linearen Regressionslinie entspricht dabei dem strength-duration time constant. Die Abbildung zeigt einen ähnlichen strength-duration time constant in beiden Gruppen (a) ( $p=0.07437$ ). (B) Recovery cycle von Patienten mit BFCS verglichen mit einer gesunden Kontrollgruppe. Es zeigten sich keine signifikanten Unterschiede des Schwellenwertes zwischen beiden Gruppen zu unterschiedlichen Zeitpunkten nach einem supramaximalen Reiz. 


\subsubsection{Recovery cycle}

Der recovery cycle, also die Veränderungen der Erregbarkeit nach einem supramaximalen Reiz, ist in Abbildung 14B dargestellt und gibt Informationen über die Länge der relativen Refraktärzeit und die Phasen der gesteigerten (superexcitability) und der verminderten (subexcitability) Erregbarkeit. Hier ergaben sich keine signifikanten Unterschiede in den beiden Gruppen. Die relative Refraktärzeit wurde berechnet aus dem ersten Schnittpunkt der Kurve mit der x-Achse und lag bei beiden Gruppe etwa bei 3 ms (BFCS=3.004x|/1.04 $\mathrm{ms}, \mathrm{NC}=3.157 \times \mid / 1.04 \mathrm{~ms}, \mathrm{p}=0.36532)$ (s. Abb. 14B).

\subsection{Reproduzierbarkeit der Messung}

Um die Reproduzierbarkeit der Ergebnisse zu überprüfen, wurden einige Probanden mehrfach der Messung unterzogen. Dabei wurde zum Einen die Messung zweimal hintereinander durchgeführt ohne die Elektroden erneut zu kleben und zum Anderen wurden Probanden nach mehreren Wochen erneut der Exzitabilitätsmessung unterzogen. In Abbildung 15 sind die Daten zweier solcher Messungen bei einem Probanden mit einem BFCS aufgetragen. Es bestehen nur minimale Unterschiede zwischen den beiden Messungen, die 11 Wochen auseinander liegen. 

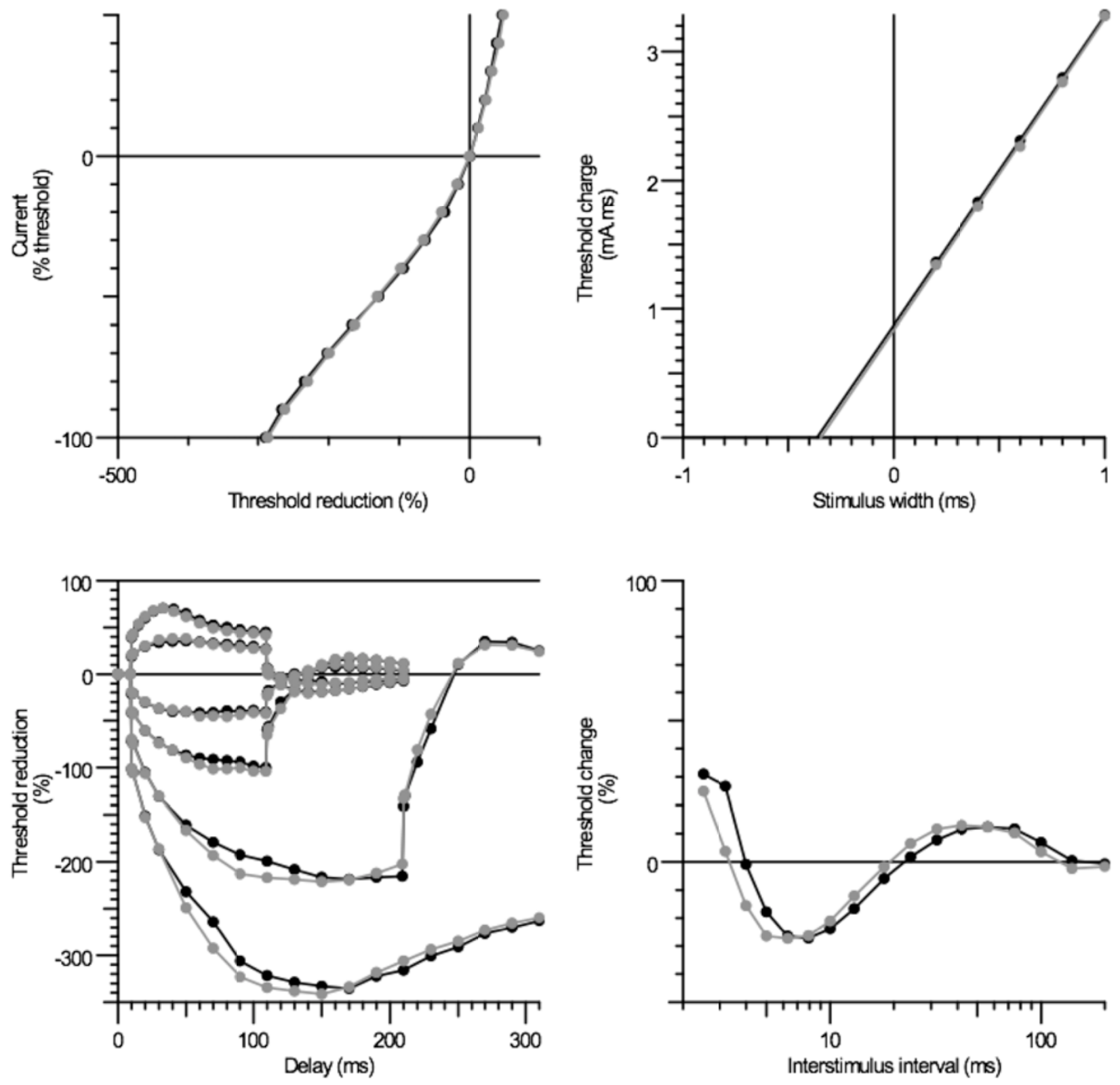

Abbildung 15 Reproduzierbarkeit der Messung: ein Proband wurde zwei mal am rechten N. medianus gemessen (schwarz: Messung vom 06.03.2013, grau: Messung vom 22.5.2013); oben links: Current/threshold relationship, oben rechts: strength-duration time, unten links: threshold electrotonus, unten rechts: recovery cycle. 


\subsection{Die wichtigsten Ergebnisse im Überblick}

Abbildung 16 zeigt die wichtigsten Ergebnisse zur Übersicht noch einmal in einem Punktdiagramm. Deutlich wird hier, dass die einzelnen Messwerte innerhalb der beiden Gruppen relativ weit um den Mittelwert herum schwanken und sich die Werte zwischen den beiden Gruppen überschneiden, auch wenn der Mittelwert und der Standardfehler signifikant unterschiedlich sind. Somit kann anhand der Messung eines Patienten mit dem Verdacht auf ein BFCS mittels threshold tracking diese Diagnose mit Hilfe der Daten dieser Arbeit nicht eindeutig verifiziert bzw. verworfen werden.

In der Abbildung 16A wird deutlich, dass die Schwellenwertzunahme bei den BFCSPatienten zwischen 90 und 100 ms nach dem konditionierenden Reiz mit einer Stärke von $-40 \%$ des Kontrollimpulses signifikant geringer war, verglichen mit einer gesunden Kontrollgruppe (s. auch Abb. 11B). Genauso sah es nach einem konditionierenden Reiz von $-70 \%$ (Abb. 16B) und -100\% (Abb. 16C) des Kontrollimpulses aus (s. auch Abb. 12). Zusammenfassend lässt sich demnach sagen, dass das Ausmaß der Schwellenwertänderungen insbesondere nach hyperpolarisierenden konditionierenden bei den BFCS-Patienten deutlich geringer war.

In der current/threshold relationship fiel auf, dass die kleinste Steigung der Patienten mit einem BFCS signifikant größer war als bei der Kontrollgruppe (Abb. 16D und s. auch 13). 
A

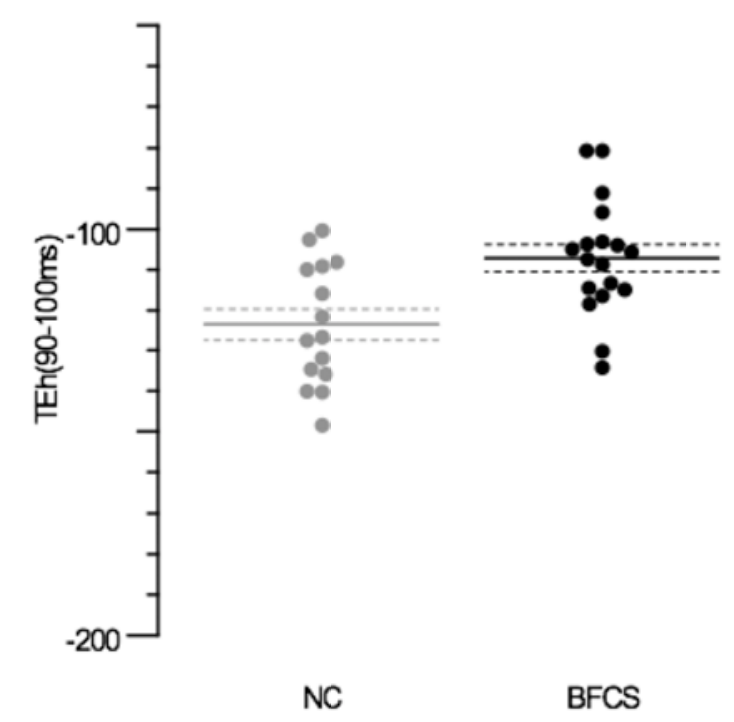

C

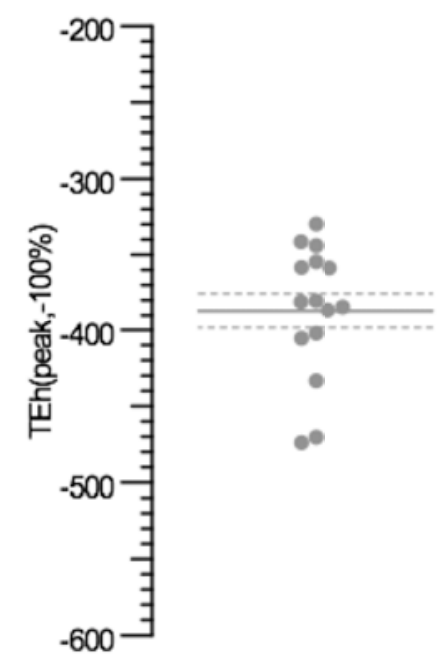

NC

BFCS
B

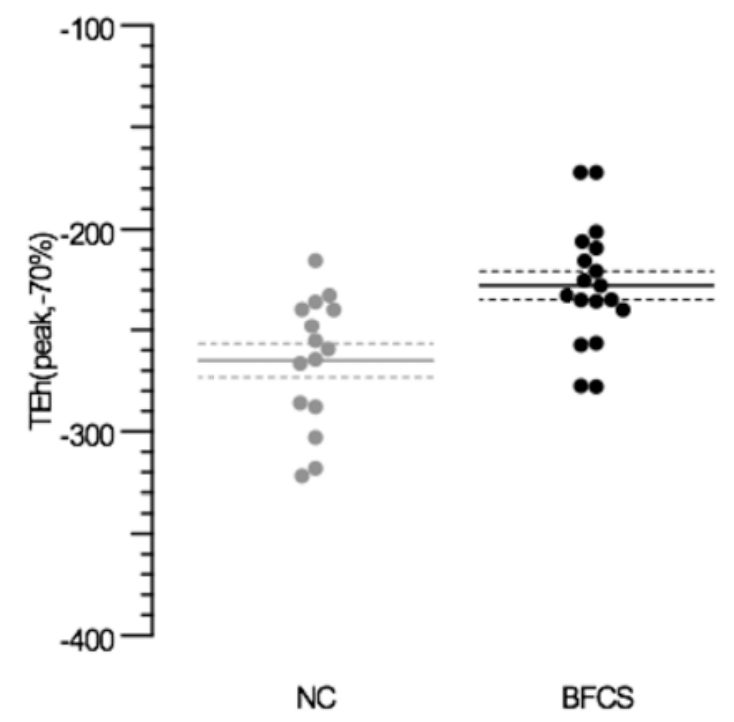

D

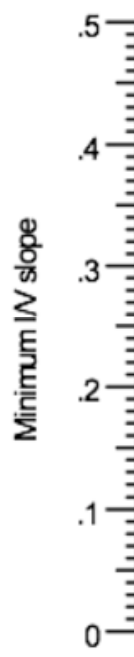

NC

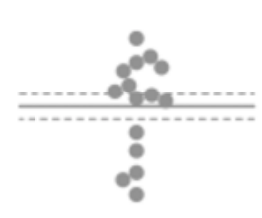

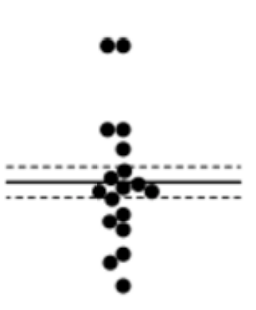

BFCS

Abbildung 16 Zusammenfassende Darstellung der wichtigsten Ergebnisse mittels eines Punktdiagrammmes. Die durchgezogene Linie gibt jeweils den Mittelwert, die gestrichelte den Standardfehler an. Jeder Punkt steht für das Messergebnis eines Probanden, wobei grau die Ergebnisse der Kontrollgruppe (NC) und schwarz die der Patienten mit einem BFCS darstellt. Zu sehen ist der signifikante Unterschied nach der Messung von (A) -40\% im threshold electrotonus 90 bis 100 ms nach dem Reiz, (B) maximale Schwellenwertzunahme nach einem $-70 \%$ und (C) $-100 \%$ starken konditionierenden Vorimpuls. (D) Signifikant größere Steigung der BFCS-Patienten am niedrigsten Punkt der I/V-Kurve. 


\section{Diskussion}

Die Ergebnisse der vorliegenden Arbeit zeigen zum ersten Mal, dass Patienten, die unter einem BFCS leiden, eindeutige Anzeichen einer axonalen Übererregbarkeit aufweisen. Die Auffälligkeiten der untersuchten Exzitabilitätsparameter deuten am ehesten auf eine veränderte inward rectification hin. Des Weiteren scheint auch eine Veränderung der langsamen $\mathrm{K}^{+}-$Kanäle eine Rolle zu spielen.

\subsection{Veränderungen der axonalen Erregbarkeit bei Patienten mit einem benignen Faszikulations-Crampus-Syndrom}

Die Veränderungen der axonalen Erregbarkeit, insbesondere die geringere Schwellenwertzunahme nach hyperpolarisierenden konditionierenden Reizen mit einer Stärke von $-70 \%$ und $-100 \%$ des Kontrollimpulses und der steilere Verlauf der I/V-Kurve ebenfalls nach hyperpolarisierenden Reizen bei den BFCS-Patienten sprechen für einen signifikant erhöhten $\mathrm{I}_{\mathrm{H}}$-Strom des Motoaxons, wenn die Daten mit einer gesunden Kontrollgruppe verglichen werden.

Die geringe Schwellenwertzunahme im threshold electrotonus nach hyperpolarisierenden Reizen kann durch einen vermehrten $\mathrm{I}_{\mathrm{H}}$-Strom entstehen, da dieser Strom die Hyperpolarisation limitiert (Pape 1996). Dieses Phänomen lässt sich am deutlichsten nach hyperpolarisierenden konditionierenden Reizen ab - $40 \%$ des Kontrollimpulses sehen, da die Kanäle durch Hyperpolarisation aktiviert werden, und ist am deutlichsten nach langen hyperpolarisierenden Reizen, da es zu einer langsamen Anpassung der Kurve durch den $\mathrm{I}_{\mathrm{H}}$-Strom kommt (Yang et al. 2001, Tomlinson et al. 2010a, Howells et al. 2012). Daher lieferte diese Arbeit die signifikantesten Ergebnisse nach einem konditionierenden Reiz von $-70 \%$ mit einer Länge von 200 ms und von $-100 \%$ des Kontrollimpulses mit 300 ms. Ein stärker ausgeprägter $I_{\mathrm{H}}$-Strom hat einen größeren Effekt auf die Daten nach einer Hyperpolarisation als nach einer Depolarisation im threshold electrotonus (Pape 1996), was die Ergebnisse der vorliegenden Arbeit bestätigen. Die einwärts-gleichrichtenden Kanäle lassen sich durch $\mathrm{Cs}^{+}$blockieren (Pape 1996, Yang et al. 2001, Krishnan et al. 2009). Die Blockade mittels $\mathrm{Cs}^{+}$zeigte tierexperimentell eine ausgedehntere Kurve in hyperpolarisierender Richtung vor allem nach dem konditionierenden Reiz mit einer Stärke 
von mindestens $-40 \%$ des Kontrollimpulses (Baker et al. 1987, Yang et al. 2001). In dieser Arbeit zeigte sich in hyperpolarisierender Richtung eine weniger ausgedehnte Kurve, was dementsprechend für eine erhöhte Aktivität der HCN-Kanäle spräche. Der steilere Verlauf der I/V- Kurve nach hyperpolarisierenden Reizen ist ein weiterer Hinweis auf einen erhöhten $\mathrm{I}_{\mathrm{H}}$-Strom (Pape 1996, Kiernan et al. 2000).

Während des Ruhemembranpotentials sind normalerweise zwischen 10 und 15\% der HCN-Kanäle aktiv und sind somit sehr wahrscheinlich an der Regulation des Ruhemembranpotentials und der Erregbarkeit eines Nerven beteiligt (Pape 1996, Biel et al. 2009, Krishnan et al. 2009). Eine vermehrte Aktivität der Kanäle hat daher auch zur Folge, dass mehr von ihnen während des Ruhemembranpotentials geöffnet sind, zu einer leichten Depolarisation der Membran führen und sich das Membranpotential näher zu dem Schwellenwert verschiebt, an dem ein Aktionspotential ausgelöst wird (Pape 1996). Die Patienten mit einem BFCS zeigten die Tendenz zu geringeren Reizintensitäten, um die gleiche Antwort des Muskels wie die Kontrollgruppe auszulösen, was eine niedrigere Schwelle zur Auslösung von Aktionspotentialen wahrscheinlich macht und als eine Ursache für die gesteigerte Aktivität der Motoneurone der Patienten, die sich in Form von Muskelkrämpfen und Faszikulationen äußert, in Frage kommt. Allerdings war dieser Unterschied zwischen beiden Gruppen mit einem p-Wert von knapp unter 0,05 nur grenzwertig signifikant und somit lässt sich hier lediglich ein Trend zu geringeren Reizintensitäten feststellen. Eine Depolarisation des Nerven führt außerdem zu Veränderungen des threshold electrotonus, indem sie die Veränderungen sowohl nach Deals auch nach Hyperpolarisation in ihrem Ausmaß verkleinert (Bostock et al. 1998, Yang et al. 2001). Diese Veränderungen ließen sich in dieser Arbeit ebenfalls beobachten, wobei nach depolarisierenden Reizen lediglich die Antwort auf den $+40 \%$ konditionierenden Impuls vermindert war. Demnach führt eine vermehrte Aktivität des $I_{H}$-Stroms zum einen zu einer Begrenzung der Hyperpolarisation und somit zu einem geringeren Ausmaß der Kurve nach negativen Reizen und zum anderen zu einer leichten Depolarisation, was wiederum das Ausmaß der Kurve nach positiven Reizen begrenzt. Die Depolarisation des Membranpotentials scheint dabei allerdings nur sehr gering zu sein, da weder eine Veränderung der Antwort nach 90-100 ms des 40\%-konditionierenden Reizes im threshold electrotonus noch der Phase der Übererregbarkeit während des recovery cycles beobachtet wurden, welche die sensitivsten Parameter für Polarisation der Membran 
darstellen (Kiernan und Bostock 2000).

Die vermehrte inward rectification bei Patienten mit einem BFCS könnte durch eine vermehrte Expression einer oder mehrerer der HCN-Isoformen zu Stande kommen oder durch eine veränderte Kinetik dieser Kanäle entstehen. So wäre es denkbar, dass die HCN-Kanäle, die ja spannungsabhängig sind, bereits bei weniger negativen Membranpotentialen öffnen. Des Weiteren wäre auch eine zentrale Modulation denkbar, die zum Beispiel über cAMP oder PIP2 die Funktion der HCN-Kanäle beeinflussen könnte.

Zwei Ergebnisse des threshold trackings, die diese Arbeit hervorgebracht hat, lassen sich allerdings nicht mit einer vermehrten inward rectification erklären. Zum Einen die geringere maximale Schwellenwertzunahme am Ende des $40 \%$-depolarisierenden Stroms $\left(=\mathrm{TEd}^{40}\right.$ undershoot) und zum Anderen die geringere maximale Schwellenwertabnahme am Ende des $40 \%$-hyperpolarisierenden Stroms ( $=\mathrm{TEd}^{40}$ overshoot). Kiernan und Kollegen fanden in 2001 bei der Untersuchung von acht Patienten, die an einer Neuromytonie litten, ebenfalls Veränderungen des $\mathrm{TEd}^{40}$ under- und overshoots, allerdings zeigte sich hier sowohl ein vergrößerter under- als auch overshoot im Vergleich zu der Kontrollgruppe (Kiernan et al. 2001b). Diese Beobachtung führten sie auf eine gesteigerte Aktivität der langsamen $\mathrm{K}^{+}-$Kanäle zurück und somit könnte dem entgegengesetzt bei den BFCSPatienten eine verminderte Aktivität dieser Kanäle eine Rolle spielen. Die langsamen $\mathrm{K}^{+}-$ Kanäle bewirken normalerweise ein kleines hyperpolarisierendes Nachpotential am Ende eines Aktionspotentials und vermindern dadurch die neuronale Erregbarkeit (Krishnan et al. 2009). Somit würde eine verminderte Aktivität zu einer Übererregbarkeit führen, die sich zum Beispiel in Form von Muskelkrämpfen äußern könnte. Allerdings waren die beiden anderen Exzitablitätsparameter, die späte Phase der Untererregbarkeit im recovery cycle und die $\mathrm{TEd}^{40}$-Accomodation, die abhängig von der Funktion der langsamen $\mathrm{K}^{+}-\mathrm{Kanäle}$ sind, bei den BFCS-Patienten nicht signifikant verändert. Daraus folgend scheint die veränderte Aktivität der langsamen $\mathrm{K}^{+}-$Kanäle nur minimal zu sein und dementsprechend wohl auch keine herausragende Rolle in der Entstehung des BFCS zu spielen. 


\subsection{Ergebnisse im Zusammenhang bisheriger Studien zum benignen Faszikulations-Crampus-Syndrom}

Die bisherigen Publikationen zur Entstehung der Symptome des BFCS haben sich vor allem auf das Vorliegen von Antikörpern gegen VGKC konzentriert. Es konnten allerdings nur in etwa einem Viertel der Patienten, die sich klinisch mit den Symptomen des BFCS präsentierten, Antikörper gegen diese Kanäle nachgewiesen werden (Liewluck et al. 2014). Dennoch ist dies sehr viel öfter der Fall als in gesunden Kontrollgruppen oder Patienten mit neurologischen Erkrankungen, die nicht immunologisch vermittelt sind (Klein et al. 2013, Liewluck et al. 2014). Daher scheinen diese Antikörper zumindest bei einem Teil der Patienten eine Rolle in der Entstehung der Symptome zu spielen. Untersuchungen bei Patienten mit einer erworbenen Neuromyotonie konnten keine Veränderungen der Exzitabilität von peripheren Nerven nachweisen, die sich durch die Einwirkung von Antikörpern gegen VGKC erklären ließen (Kiernan et al. 2001b). Es fehlen allerdings noch Studien zur peripheren Erregbarkeit des Nerven mit einer größeren Anzahl von Patienten, bei denen der Antikörpernachweis positiv ist und die sich klinisch mit Faszikulationen und Muskelkrämpfen unklarer Genese präsentieren.

Interessant wäre demnach für die Patienten mit einem BFCS ein Vergleich der Exzitabilitätsparameter von seropositiven Patienten für Antikörper gegen VGKC mit seronegativen. Dabei sollte beachtet werden, dass auch die konditionierenden Reize mit $-70 \%$ und $-100 \%$ des Kontrollimpulses in das Protokoll integriert werden, da diese Werte am deutlichsten durch eine Veränderung in der Kinetik oder Expression von HCN-Kanälen verändert werden (Howells et al. 2012). Innerhalb einer solchen Studie könnten sich evtl. Subgruppen des BFCS abzeichnen bzw. ein fließender Übergang zur erworbenen Neuromyotonie und insbesondere dem Isaac-Syndrom deutlich werden.

Diese Arbeit gibt zum ersten Mal einen Hinweis darauf, dass möglicherweise eine Veränderung der HCN-Kanäle maßgeblich an der Entstehung des BFCS beteiligt sein könnte. Zukünftige Arbeiten sollten sich demnach nicht ausschließlich auf die Rolle der $\mathrm{K}^{+}$Kanäle konzentrieren, sondern ihren Fokus insbesondere auf die HCN-Kanäle richten. 


\subsection{Vergleich der Ergebnisse zu anderen Erkrankungen mit Faszikulationen und Muskelkrämpfen}

\subsubsection{Amyotrophe Lateralsklerose (ALS)}

Die amyotrophe Lateralsklerose ist eine progressive neurodegenerative Erkrankung, die die Motoneuronen des zentralen Nervensystems betrifft. Patienten leiden häufig als erste Symptome unter Faszikulationen und Muskelkrämpfen. Daher ist die ALS gerade zu Beginn eine wichtige Differentialdiagnose zum BFCS. Untersuchungen der peripheren Nerven haben einen verlängerten strength-duration time constant und eine kleinere Rheobase ergeben, was durch einer Erhöhung des persistierenden $\mathrm{Na}^{+}$-Stroms erklärbar wäre (Mogyoros et al. 1998, Krishnan et al. 2009). Die Veränderungen im threshold electrotonus variieren innerhalb der Krankheitsstadien. In frühen Stadien zeigt sich eine Verminderung der $\mathrm{K}^{+}$-Leitfähigkeit, die sich durch größere Schwellenwertänderungen nach langen depolarisierenden Reizen auszeichnet (Kanai et al. 2006, Krishnan et al. 2009). In fortgeschritteneren Stadien kommt zu der Reduzierung der $\mathrm{K}^{+}$-Leitfähigkeit eine Erhöhung des persistierenden $\mathrm{Na}^{+}$-Stroms hinzu, die sich in verminderten Schwellenwertänderungen als Antwort auf hyperpolarisierende Reize äußert (Krishnan et al. 2009, Bae et al. 2013).

Sowohl die gesteigerte $\mathrm{Na}^{+}$- als auch die verminderte $\mathrm{K}^{+}$-Leitfähigkeit führen $\mathrm{zu}$ einer Depolarisation der Membran, ebenso wie ein größerer Strom durch die HCN-Kanäle. Die Ursache für Faszikulationen und Muskelkrämpfe bei Patienten mit ALS bzw. BFCS könnte demnach ein leicht depolarisiertes Ruhemembranpotential zu sein, welches allerdings durch verschiedene lonenkanal-Veränderungen entsteht. Allerdings scheint bei der ALS auch eine kortikale Übererregbarkeit vorhanden zu sein (Menon et al. 2014). Untersuchungen zur kortikalen Übererregbarkeit beim BFCS wurden bis jetzt noch nicht durchgeführt. 


\subsubsection{Neuromyotonie}

Die charakteristischen Symptome der Neuromyotonie sind Myokymien (Muskelwogen), schmerzhafte Muskelkrämpfe und Muskelsteife (Zierz 2003). Sind diese Symptome im Rahmen eines autoimmunologischen Geschehens erworben worden, so spricht man von einem Isaac-Syndrom oder, wenn Symptome der zentralen oder autonomen Übererregbarkeit zusätzlich auftreten, von einem Morvan-Syndrom (Fischer und Schröder 2004, Vucic et al. 2010). Ursache dieser Erkrankungen scheinen Autoantikörper gegen spannungsabhängige $\mathrm{K}^{+}-$Kanäle zu sein (Hart et al. 2002, Kleopa et al. 2006).

Studien haben gezeigt, dass die Blockade der schnellen $\mathrm{K}^{+}$-Kanäle durch 4-Aminopyridin die Phase der Übererregbarkeit verlängert, die frühe Depolarisation im threshold electrotonus ansteigt und es zu einer leichten Depolarisation der Membran kommt (Baker et al. 1987, Yang et al. 2000).

1999 zeigte eine Studie von Maddison und Kollegen eine Verlängerung des strengthduration time constants bei neun Patienten mit einer erworbenen Neuromytonie im Vergleich zu neun gesunden Probanden (Maddison et al. 1999). Diese Beobachtung könnte für eine Depolarisation der Membran sprechen, einer Erhöhung der $\mathrm{Na}^{+}-$ Leitfähigkeit oder eine paranodale Demyelinisierung bedeuten (Maddison et al. 1999, Kiernan et al. 2001b). Möglich wäre auch, dass die VGKC-Antikörper einen indirekten Effekt auf die Membran haben und so ebenfalls an der Veränderung des strength-duration time constants beteiligt sind (Maddison et al. 1999, Kiernan et al. 2001b). Wie bereits beschrieben, konnte auch bei Patienten, die an einer ALS erkrankt sind, eine Verlängerung des strength-duration time constants beobachtet werden (Mogyoros et al. 1998, Krishnan et al. 2009). Diese Arbeit zeigt einen normalen strength-duration time constant bei den BFCS-Patienten.

Kiernan und Kollegen haben 2001 versucht, mittels des threshold trackings diese Unterschiede in der Erregbarkeit zwischen Patienten mit einer erworbenen Neuomyotonie und gesunden Probanden darzustellen. Sie konnten die charakteristischen Veränderungen nicht nachweisen, die durch eine Blockade der VGKC zu erwarten wären, allerdings wiesen auch nur drei der gemessen Patienten mit einer Neuromyotonie Antikörper gegen diese Kanäle auf. Auch eine Verlängerung des strength-duration time constant konnte nicht beobachtet werden. Sie fanden allerdings eine verlängerte späte Phase der Unter- 
erregbarkeit des recovery cycles, größere Akkommodation nach Depolarisation im threshold electrotonus und einen höheren over- und undershoot, was am ehesten für eine vermehrte Aktivität der langsamen $\mathrm{K}^{+}$-Kanäle spricht und möglicherweise eine Anpassung an die dauerhafte Aktivität der motorischen Einheit darstellt (Kiernan et al. 2001b). Wie bereits beschrieben, zeigte diese Arbeit einen geringeren over- und undershoot bei den untersuchten BFCS-Patienten, was demzufolge für eine leicht verringerte Aktivität der langsamen $\mathrm{K}^{+}$-Kanäle sprechen könnte. Veränderungen der inward rectification konnten Kiernan und Kollegen nicht feststellen, allerdings wäre hierfür das erweiterte TrondProtokoll mit stärker hyperpolarisierenden Reizen aussagekräftiger und somit könnten bestehende Veränderungen in der vorliegenden Studie übersehen worden sein.

\subsection{Ist die veränderte inward rectification die Ursache oder das Ergebnis einer peripheren Übererregbarkeit?}

Die vermehrte inward rectification, die bei den BFCS-Patienten beobachtet wurde, könnte zum einen die Ursache für die Faszikulationen und Muskelkrämpfe sein, es wäre allerdings auch denkbar, dass die vermehrte Aktivität der motorischen Einheiten zu einer Hochregulierung der HCN-Kanäle führt, um einen Leitungsblock zu verhindern. Um diese Zusammenhänge genauer zu beleuchten, soll nun im Folgenden die Ergebnisse des threshold trackings an sensiblen Fasern und an low-threshold Motoaxonen im Vergleich zu den Veränderungen der BFCS-Patienten, die diese Arbeit zeigt, zusammenfassend dargestellt und die pathophysiologische Rolle der HCN-Kanäle für weitere Erkrankungen erläutert werden.

\subsubsection{Bedeutung der inward rectification sensibler Neurone im Vergleich zu den Ergebnissen dieser Arbeit}

Viele Untersuchungen haben bereits gezeigt, dass sensible Axone verglichen mit Motoaxonen einen verstärkte Expression der einwärts-gleichrichtenden Kationenkanäle besitzen (Bostock und Bergmans 1994, Lin et al. 2002, Krishnan et al. 2009). Sie zeigen, verglichen mit Motoaxonen, ein ähnliches Erscheinungsbild im threshold electrotonus mit einem geringeren Ausmaß der Kurven vor allem nach hyperpolarisierenden Reizen und in 
der stimulus-response-curve, die nach links verschoben ist, wie die Patienten mit BFCS verglichen mit der gesunden Kontrollgruppe in dieser Arbeit (Trevillion et al. 2007, Krishnan et al. 2009). Darüber hinaus haben sensorische Axone einen längeren strengthduration time constant und eine kleinere Rheobase (Mogyoros et al. 1996, 1998, Bostock und Rothwell 1997, Lin et al. 2002). Diese Veränderungen führen Bostock und Rothwell auf mehr persistierende $\mathrm{Na}^{+}$-Kanäle, die verzögert auf Depolarisation des Membranpotentials aktivieren und auch nicht sofort wieder inaktivieren, in sensorischen Axonen zurück (Bostock und Rothwell 1997). Allerdings liegen noch keine Daten darüber vor, wie viele der $\mathrm{Na}^{+}$-Kanäle sich tatsächlich im persistierenden Modus befinden und die Autoren können auch nicht ausschließen, dass die sensorischen Axone depolarisierter sind als die motorischen (Bostock und Rothwell 1997). Neuere Untersuchungen ziehen auch eine veränderte Kinetik von $\mathrm{K}^{+}$-Kanälen in Betracht, die ihrerseits zu einer Depolarisation der Membran führen würden und dementsprechend die beobachteten Veränderungen erklären könnten (Howells et al. 2012). Weder Veränderungen des strength-duration time constants noch der Rheobase konnten bei den BFCS-Patienten beobachtet werden. Daher ist davon auszugehen, dass in dieser Patientenpopulation nur die veränderte inward rectification eine Rolle spielt.

Sensible Axone neigen eher zu ektopischen Entladungen und leiten auch eine längere Folge von Impulsen weiter ohne einen Leitungsblock zu entwickeln (Bostock et al. 1991a, 1991b, Bostock und Bergmans 1994, Kiernan et al. 1997, Vagg et al. 1998). Daraus lässt sich schließen, dass eine vermehrte inward rectification zu einer erhöhten Erregbarkeit führt und somit auch zu einer Häufung von ektopischen Entladungen, die sich an sensorischen Nerven in Form von Schmerz und Parästhesien äußern kann (Chaplan et al. 2003), in Motoneuronen hingegen in Muskelkrämpfen und Faszikulationen. Die gesteigerte inward rectification könnte demnach auch an der Aufrechterhaltung eines Muskelkrampfes beteiligt sein, da sie durch das Verhindern eines Leitungsblockes die Weiterleitung der hochfrequenten Signale während des Krampfes ermöglicht. Mathematische Berechnungen haben gezeigt, dass die unterschiedlichen Antworten von motorischen und sensorischen Axonen auf hyperpolarisierende Reize am ehesten durch Unterschiede der spannungsabhängigen Aktivierung von schnellen HCN-Isoformen, mutmaßlich HCN1, entsteht (Howells et al. 2012). Es wäre somit denkbar, dass Patienten mit einem BFCS eine gesteigerte Expression bzw. eine veränderte Kinetik der HCN1-Isoform besitzen. 


\subsubsection{Vergleich der Ergebnisse mit low-threshold Motoaxonen}

Innerhalb von Motoaxonen gibt es Unterschiede in ihrer Erregbarkeit: es gibt Motoaxone mit einem niedrigen Schwellenwert für die Auslösung eines Aktionspotentials ( $=$ lowthreshold) und solche mit einem hohen (=high threshold) (Henneman et al. 1965, Trevillion et al. 2010). Trevillion hat in seiner Untersuchung gezeigt, dass die low-threshold Motoaxone im Vergleich zu den high-threshold Motoaxonen einen signifikanten Unterschied des $\mathrm{I}_{\mathrm{H}}$-Stroms zeigten (Trevillion et al. 2010). Die beobachteten Veränderungen im Trond-Protokoll entsprachen dabei denen der BFCS-Patienten in dieser Arbeit. Es wurden keine signifikanten Unterschiede zwischen low- und high-threshold Motoaxonen in der strength-duration time constant festgestellt (Trevillion et al. 2010). Nach mathematischen Berechnungen ergab sich eine fast doppelt so große hyperpolarisationsaktivierte Leitfähigkeit für den $\mathrm{I}_{\mathrm{H}}$-Strom (Trevillion et al. 2010).

Eine weitere Beobachtung von Trevillion und seinen Kollegen war, dass die meisten der low-threshold Axone einen relativ großen Durchmesser besitzen und sich diese Art von Neuronen durch eine höhere Entladungsrate und die Weiterleitung von hochfrequenten Ladungsimpulse auszeichnen (Trevillion et al. 2010). Denkbar wäre demnach, dass die BFCS-Patienten mehr dieser low-threshold Axone besitzen und bereits Reize einer geringen Intensität zu vielen Entladungen führen, die sich dann in Faszikulationen und Muskelkrämpfen äußern.

\subsubsection{Pathophysiologische Rolle der axonalen HCN-Kanäle}

In den letzten Jahren wurde bei einigen Erkrankungen eine Veränderung der HCN-Kanäle festgestellt. An diesen Ergebnissen wird deutlich, dass die Rolle der HCN-Kanäle in der Entstehung bzw. der Ausprägung von Symptomen in den unterschiedlichsten Erkrankungen bislang unterschätzt wurde. In Zukunft könnten sich durch die genauere Untersuchung dieser Kanäle neue Einblicke in die Pathophysiologie vieler Erkrankungen und somit auch eine neuer Ansatz zur Entwicklung von Therapiestrategien ergeben.

Viele Untersuchungen bezogen sich auf die Veränderungen bei diabetischer Polyneuropathie. Tierexperimentell wurde 2001 eine verminderte axonale inward rectification festgestellt (Yang et al. 2001), die die Ergebnisse aus Untersuchungen am Menschen aus 1996 belegten (Horn et al. 1996). Andere Studien sehen allerdings eher die Ursache in 
einer Dysfunktion der $\mathrm{Na}^{+}-\mathrm{K}^{+}-$Pumpe, die $\mathrm{zu}$ einer Depolarisation des Nerven führt (Krishnan und Kiernan 2005, Krishnan et al. 2008). Der genaue Pathomechanismus bleibt demnach weiterhin unklar.

Jankelowitz und Kollegen fanden 2007 heraus, dass Patienten mit einer Hemiplegie nach einem Schlaganfall auf der plegischen Seite eine signifikant geringere inward rectification zeigten, verglichen mit der anderen Seite und einer Kontrollgruppe. Sie folgerten daraus, dass chronische Beeinträchtigungen im kortikospinalen Trakt zu einer Verminderung des $\mathrm{I}_{\mathrm{H}}$-Stroms führen (Jankelowitz et al. 2007a).

2008 wurde eine verminderte inward rectification bei Patienten festgestellt, die an einer akut intermittierenden Porphyrie (AIP) litten, aber keine Zeichen einer Neuropathie zeigten (Lin et al. 2008). Ein Patient mit einer AIP und einer Neuropathie zeigte eine Dysfunktion der $\mathrm{Na}^{+}-\mathrm{K}^{+}-\mathrm{Pumpe}$ (Lin et al. 2008). Demnach könnte die verminderte inward rectification ein subklinisches Symptom des beeinträchtigten metabolischen Stoffwechsels sein sowohl bei Patienten mit einer AIP (Lin et al. 2008) als auch mit einer diabetischen Stoffwechsellage und erst bei einer manifesten Neuropathie kommt es zu Beeinträchtigungen der $\mathrm{Na}^{+}-\mathrm{K}^{+}-\mathrm{Pumpe}$.

Viele Arbeiten konzentrierten sich auf die Ursache von neuropathischem Schmerz. Dieser Schmerz ist gekennzeichnet durch spontan auftretende Schmerzen, Hyperalgesie und Allodynie und kann im Rahmen vieler Erkrankungen und Traumen entstehen (Campbell und Meyer 2006, Biel et al. 2009). Ein Kennzeichen dieser Erkrankung ist die Entstehung von abnormalen spontanen (ektopischen) Entladungen (Biel et al. 2009). Einige Untersuchungen legen einen kausalen Zusammenhang zwischen einer vermehrten Aktivität von HCN-Kanälen und der Entstehungen von Neuralgien nahe (Chaplan et al. 2003, Yao et al. 2003). Dabei scheinen vor allem die Isoformen HCN1 und HCN2 eine Rolle zu spielen (Biel et al. 2009). Diese Ergebnisse legen die Vermutung nahe, dass ein vermehrter $\mathrm{I}_{\mathrm{H}}$-Strom $\mathrm{Zu}$ einer gesteigerten Erregbarkeit führt und verantwortlich für ektopische Entladungen ist (Biel et al. 2009). Demnach wäre es möglich, dass ein gesteigerter $\mathrm{I}_{\mathrm{H}}$-Strom an sensorischen Axonen zu Schmerzen und an motorischen Axonen zu Faszikulationen und Muskelkrämpfen im Rahmen des BFCS führen kann. 


\subsection{Schlussfolgerung und Ausblick}

Im Rahmen dieser Arbeit konnte gezeigt werden, dass das threshold tracking eine gute Methode ist, Informationen über die axonale Erregbarkeit und die zugrundeliegenden Funktionen der lonenkanäle zu erhalten. Die Methode war dabei relativ einfach und in kurzer Zeit durchführbar. Daher erscheint es sinnvoll, das threshold tracking weiter zu verfeinern und in den klinischen Alltag zu integrieren. Mithilfe einer großen Datenbank für eine gesunde Kontrollgruppe, die nach dem Alter sortiert sein müsste, könnten die Ergebnisse der Messung bei den Patienten dann interpretiert werden. Dazu wurden bereits 105 gesunde Probanden untersucht, auch hinsichtlich des Effektes von Alter, Geschlecht und BMI auf die Exzitabilitätsparameter (McHugh et al. 2011). Problematisch, insbesondere für die Untersuchung der Funktion der HCN-Kanäle, scheint allerdings zu sein, dass die Antworten auf hyperpolarisierende Reize und dabei speziell das Minimum der Steigung der I/V-Kurve, TEh ${ }^{100}$ (peak) und $\mathrm{TEh}^{70}$ (peak) sich am deutlichsten von allen gemessen Exzitabilitätsparametern zwischen gesunden Personen unterschieden (Tomlinson et al. 2010a). Dadurch wird es nur schwer möglich sein, zuverlässige Referenzwerte zur Unterscheidung zwischen gesunden Personen und Patienten mit einer veränderten Expression oder Kinetik der HCN-Kanäle festzulegen.

Die beobachteten Veränderungen der Exzitabilitätsparameter mit einer geringeren Abnahme des Schwellenwertes auf hyperpolarisierende konditionierende Reize und einer steileren I/V-Kurve in hyperpolarisierender Richtung sind am ehesten auf eine veränderte Expression oder Kinetik der HCN-Kanäle zurückzuführen. Das mathematische Modelling der Daten, welches noch exakter die zugrundeliegenden Veränderungen analysieren kann, steht noch aus. Zukünftige Studien sollten sich demnach auf die genauere Untersuchung dieser Kanäle konzentrieren. Über die Verteilung der Isoformen am peripheren Nerven des Menschen ist noch nicht viel bekannt, und auch die Untersuchung der exakten Funktion, Regulierung über cAMP und anderer Mechanismen sowie die pathologische Rolle dieser Kanäle steht noch am Anfang. Daher könnten zukünftige Studien über die genauen Veränderungen der HCN-Kanäle bei Patienten mit einem BFCS nicht nur einen großen Schritt zum Verständnis dieser Erkrankung bedeuten, sondern generell Aufschluss über die Funktion an peripheren Nerven des Menschen bringen. Ein besonderes Augenmerk sollte dabei auch auf zentrale Regulierungsmechanismen gelegt werden, da es ebenfalls möglich wäre, dass zentrale Mechanismen zu einer peripheren 
Übererregbarkeit des Motoaxons bei BFCS-Pateinten führen könnten.

Da viele BFCS-Patienten vor allem über Krämpfe in den Waden und Füßen klagen und mittels des threshold trackings nur der Punkt des Nerven untersucht wird, der im Messgebiet liegt, sollte in Zukunft auch versucht werden, an der unteren Extremität diese Messung durchzuführen. Interessant wäre in diesem Zusammenhang der Vergleich von Regionen, die sehr häufig und stark von Muskelkrämpfen betroffen sind, mit denen, die selten oder nie diese Form der Übererregbarkeit zeigen. So könnten weitere Informationen gewonnen werden, ob es sich um ein generalisiertes oder fokales Syndrom handelt und ob die Schwere der Symptomatik mit den Veränderungen der Exzitabilitätsparameter korreliert. 


\section{$5 \quad$ Zusammenfassung}

Hintergrund: Der genaue Pathomechanismus zur Entstehung des benignen Faszikulations-Crampus-Syndrom ist nicht bekannt, und es wurde noch nie eine größere Anzahl dieser Patienten mittles des threshold trackings untersucht.

Material und Methoden: 18 Patienten mit einem BFCS und 15 gesunde Probanden wurden mithilfe des threshold trackings, welches eine Methode zur Messung von Exzitabilitätsparametern von peripheren Nerven in vivo ist, untersucht und die Ergebnisse verglichen.

Ergebnisse: Die stärksten Unterschiede zeigten sich im threshold electrotonus, der current/threshold relationship und der stimulus-response-curve. Im threshold electrotonus war insbesondere die Schwellenwertzunahme auf hyperpolarisierende Reize signifikant geringer bei einer Reizstärke von $-40 \%,-70 \%$ und $-100 \%$ des Kontrollimpulses $(p<0,01)$. Die current/threshold relationship war in hyperpolasierender Richtung bei den BFCSPatienten steiler als bei der gesunden Kontrollgruppe $(p<0,05)$. Außerdem benötigten sie eine deutlich geringere Reizintensität, um $50 \%$ des maximalen Summenaktionspotentials des Muskels auszulösen $(p<0,05)$.

Schlussfolgerung: Die Ergebnisse weisen auf eine veränderte Expression oder Kinetik der HCN-Kanäle an peripheren Nerven der Patienten mit einem BFCS hin und geben folglich neue Hinweise auf die Entstehung dieser Erkrankung. Da zum ersten Mal ein Zusammenhang zwischen der Veränderung der HCN-Kanäle und dem BFCS gezeigt werden konnte, sollten sich zukünftige Studien darauf konzentrieren, genauere Informationen über diese Pathologie zu erlangen. 


\section{$6 \quad$ Literaturverzeichnis}

Arbeitsgemeinschaft der Wissenschaftlichen Medizinischen Fachgesellschaften (AWMF) (2012). S1-Leitlinie: Erkrankungen der Muskulatur - Crampi/Muskelkrampf http://www.awmf.org/leitlinien/detail/l/030-037.html (aufgerufen am 12.03.2014).

Bae JS, Sawai S, Misawa S, Kanai K, Isose S, Shibuya K, Kuwabara S (2008): Effects of age on excitability properties in human motor axons. Clin Neurophysiol 119: 2282-2286

Bae JS, Simon NG, Menon P, Vucic S, Kiernan MC (2013): The Puzzling Case of Hyperexcitability in Amyotrophic Lateral Sclerosis. J Clin Neurol 9: 65-74

Baker M, Bostock H, Grafe P, Martius P (1987): Function and distribution of three types of rectifying channel in rat spinal root myelinated axons. J Physiol 383: 45-67

Barrett EF, Barrett JN (1982): Intracellular recording from vertebrate myelinated axons: mechanism of the depolarizing afterpotential. J Physiol $\underline{323}$ : 117-144

Bateman DN, Dyson EH (1986): Quinine toxicity. Adverse Drug React. Acute Poisoning Rev $\underline{5}: 215-233$

Biel M, Wahl-Schott C, Michalakis S, Zong X (2009): Hyperpolarization-activated cation channels: from genes to function. Physiol Rev $\underline{89}$ : 847-885

Blexrud MD, Windebank AJ, Daube JR (1993): Long-term follow-up of 121 patients with benign fasciculations. Ann Neurol 34: 622-625

Bodkin CL, Kennelly KD, Boylan KB, Crook JE, Heckman MG, Rubin DI (2009): Defining normal duration for afterdischarges with repetitive nerve stimulation: a pilot study. J Clin Neurophysiol Off Publ Am Electroencephalogr Soc 26: 45-49

Bostock H (1983). The strength-duration relationship for excitation of myelinated nerve: computed dependence on membrane parameters. J Physiol 341: 59-74

Bostock H, Baker M (1988): Evidence for two types of potassium channel in human motor axons in vivo. Brain Res $\underline{462}$ : 354-358 
Bostock H, Bergmans J (1994): Post-tetanic excitability changes and ectopic discharges in a human motor axon. Brain J Neurol 117 ( Pt 5): 913-928

Bostock H, Rothwell JC (1997): Latent addition in motor and sensory fibres of human peripheral nerve. J Physiol 498 ( Pt 1): 277-294

Bostock H, Sears TA, Sherratt RM (1983): The spatial distribution of excitability and membrane current in normal and demyelinated mammalian nerve fibres. $\mathrm{J}$ Physiol $\underline{341}$ : $41-58$

Bostock H, Baker M, Grafe P, Reid G (1991a). Changes in excitability and accommodation of human motor axons following brief periods of ischaemia. J Physiol 441: 513-535

Bostock H, Baker M, Reid G (1991b): Changes in excitability of human motor axons underlying post-ischaemic fasciculations: evidence for two stable states. J Physiol 441 : $537-557$

Bostock H, Cikurel K, Burke D (1998): Threshold tracking techniques in the study of human peripheral nerve. Muscle Nerve 21: 137-158

Burke D, Mogyoros I, Vagg R, Kiernan MC (1998): Quantitative description of the voltage dependence of axonal excitability in human cutaneous afferents. Brain 121: 1975-1983

Burke D, Kiernan MC, Bostock H (2001): Excitability of human axons. Clin Neurophysiol 112: $1575-1585$

Campbell JN, Meyer RA (2006): Mechanisms of neuropathic pain. Neuron 52: 77-92

Caress JB, Walker FO (2002): The spectrum of ectopic motor nerve behavior: from fasciculations to neuromyotonia. The neurologist $\underline{8}: 41-46$

De Carvalho M, Swash M (2004): Cramps, muscle pain, and fasciculations: not always benign? Neurology 63 : 721-723

De Carvalho M, Swash M (2011): Fasciculation-cramp syndrome preceding anterior horn cell disease: an intermediate syndrome? J Neurol Neurosurg Psychiatry 82: 459-461 
Chaplan SR, Guo H-Q, Lee DH, Luo L, Liu C, Kuei C, Velumian AA, Butler MP, Brown SM, Dubin AE (2003): Neuronal hyperpolarization-activated pacemaker channels drive neuropathic pain. J Neurosci 23: 1169-1178

Desai J, Swash M (1997). Fasciculations: what do we know of their significance? J Neurol Sci 152 Suppl 1: S43-48

Dias P, Terracciano CM (2013). Hyperpolarization-Activated Cyclic Nucleotide-Gated Channels and Ventricular Arrhythmias in Heart Failure: A Novel Target for Therapy? J Am Heart Assoc Cardiovasc Cerebrovasc Dis $\underline{2}$ (3): e000287

DiFrancesco D, Tortora P (1991): Direct activation of cardiac pacemaker channels by intracellular cyclic AMP. Nature 351: 145-147

Doan TN, Stephans K, Ramirez AN, Glazebrook PA, Andresen MC, Kunze DL (2004): Differential Distribution and Function of Hyperpolarization-Activated Channels in Sensory Neurons and Mechanosensitive Fibers. J Neurosci 24: 3335-3343

Fermont J, Arts IMP, Overeem S, Kleine BU, Schelhaas HJ, Zwarts MJ (2010). Prevalence and distribution of fasciculations in healthy adults: Effect of age, caffeine consumption and exercise. Amyotroph. Lateral Scler 11: 181-186

Fischer DD, Schröder R (2004): Isaac-Syndrom. Nervenarzt 75: 531-535

Harrison TB, Benatar M (2007): Accuracy of repetitive nerve stimulation for diagnosis of the cramp-fasciculation syndrome. Muscle Nerve 35: 776-780

Hart IK, Maddison P, Newsom-Davis J, Vincent A, Mills KR (2002): Phenotypic variants of autoimmune peripheral nerve hyperexcitability. Brain J Neurol 125: 1887-1895

Van der Heijden A, Spaans F, Reulen J (1994). Fasciculation potentials in foot and leg muscles of healthy young adults. Electroencephalogr Clin Neurophysiol 93: 163-168

Henneman E, Somjen G, Carpenter DO (1965): Functional significance of cell size in spinal motorneurons. J Neurophysiol $\underline{28}$ : 560-580 
Hofmann F, Biel M, Kaupp UB (2005): International Union of Pharmacology. LI. Nomenclature and Structure-Function Relationships of Cyclic Nucleotide-Regulated Channels. Pharmacol Rev 57: 455-462

Horn S, Quasthoff S, Grafe P, Bostock H, Renner R, Schrank B (1996): Abnormal axonal inward rectification in diabetic neuropathy. Muscle Nerve 19: 1268-1275

Howells J, Trevillion L, Bostock H, Burke D (2012): The voltage dependence of $\mathrm{Ih}$ in human myelinated axons. J Physiol 590: 1625-1640

Hufschmidt A, Lücking CH: Neurologie compact: Für Klinik und Praxis. 5. Auflage; Georg Thieme Verlag, Stuttgart 2009

Jankelowitz SK, Howells J, Burke D (2007a): Plasticity of inwardly rectifying conductances following a corticospinal lesion in human subjects. J. Physiol. 581: 927-940

Jankelowitz SK, McNulty PA, Burke D (2007b): Changes in measures of motor axon excitability with age. Clin Neurophysiol 118: 1397-1404

Kanai K, Kuwabara S, Misawa S, Tamura N, Ogawara K, Nakata M, Sawai S, Hattori T, Bostock H (2006): Altered axonal excitability properties in amyotrophic lateral sclerosis: impaired potassium channel function related to disease stage. Brain J Neurol 129: 953962

Keidar S, Binenboim C, Palant A (1982): Muscle cramps during treatment with nifedipine. Br Med J Clin Res Ed 285: 1241-1242

Khan SI, Burne JA (2007): Reflex Inhibition of Normal Cramp Following Electrical Stimulation of the Muscle Tendon. J Neurophysiol 98: 1102-1107

Kiernan MC, Bostock H (2000): Effects of membrane polarization and ischaemia on the excitability properties of human motor axons. Brain J Neurol 123 Pt 12: 2542-2551

Kiernan MC, Mogyoros I, Hales JP, Gracies JM, Burke D (1997): Excitability changes in human cutaneous afferents induced by prolonged repetitive axonal activity. J Physiol $\underline{500}$ (Pt 1): 255-264 
Kiernan MC, Burke D, Andersen KV, Bostock H (2000). Multiple measures of axonal excitability: a new approach in clinical testing. Muscle Nerve 23: 399-409

Kiernan MC, Cikurel K, Bostock H (2001a): Effects of temperature on the excitability properties of human motor axons. Brain J Neurol 124: 816-825

Kiernan MC, Hart I, Bostock H (2001b): Excitability properties of motor axons in patients with spontaneous motor unit activity. J Neurol Neurosurg Psychiatry $\underline{70}$ : 56-64

Klein CJ, Lennon VA, Aston PA, McKeon A, O'Toole O, Quek A, Pittock SJ (2013): Insights from LGl1 and CASPR2 potassium channel complex autoantibody subtyping. JAMA Neurol 70: 229-234

Kleopa KA, Elman LB, Lang B, Vincent A, Scherer SS (2006). Neuromyotonia and limbic encephalitis sera target mature Shaker-type $\mathrm{K}+$ channels: subunit specificity correlates with clinical manifestations. Brain J Neurol 129: 1570-1584

Krishnan AV, Kiernan MC (2005): Altered nerve excitability properties in established diabetic neuropathy. Brain 128: 1178-1187

Krishnan AV, Lin CS-Y, Kiernan MC (2008): Activity-dependent excitability changes suggest $\mathrm{Na}+/ \mathrm{K}+$ pump dysfunction in diabetic neuropathy. Brain 131: 1209-1216

Krishnan AV, Lin CS-Y, Park SB, Kiernan MC (2009). Axonal ion channels from bench to bedside: a translational neuroscience perspective. Prog Neurobiol 89: 288-313

Kuwabara Y, Kuwahara K, Takano M, Kinoshita H, Arai Y, Yasuno S, Nakagawa Y, Igata S, Usami S, Minami T et al. (2013): Increased expression of HCN channels in the ventricular myocardium contributes to enhanced arrhythmicity in mouse failing hearts. J Am Heart Assoc 24,2 (3): e000150

Kuwahara K, Saito Y, Takano M, Arai Y, Yasuno S, Nakagawa Y, Takahashi N, Adachi Y, Takemura G, Horie M et al. (2003): NRSF regulates the fetal cardiac gene program and maintains normal cardiac structure and function. EMBO J 22: 6310-6321

Lane RJ, Mastaglia FL (1978): Drug-induced myopathies in man. Lancet 8089: 562-566 
Layzer RB (1994): The origin of muscle fasciculations and cramps. Muscle Nerve 17: 1243-1249

Liewluck T, Klein CJ, Jones LK Jr (2014): Cramp-fasciculation syndrome in patients with and without neural autoantibodies. Muscle Nerve $\underline{49}$ : 351-356

Lin CS-Y, Kuwabara S, Cappelen-Smith C, Burke D (2002): Responses of human sensory and motor axons to the release of ischaemia and to hyperpolarizing currents. J Physiol 541: 1025-1039

Lin CS-Y, Krishnan AV, Lee M-J, Zagami AS, You H-L, Yang C-C, Bostock H, Kiernan MC (2008): Nerve function and dysfunction in acute intermittent porphyria. Brain 131: 25102519

Lopate G, Streif E, Harms M, Weihl C, Pestronk A (2013): Cramps and small-fiber neuropathy. Muscle Nerve $\underline{48}$ : 252-255

Maddison P, Newsom-Davis J, Mills KR (1999): Strength-duration properties of peripheral nerve in acquired neuromyotonia. Muscle Nerve 22: 823-830

Man-Son-Hing M, Wells G, Lau A (1998): Quinine for Nocturnal Leg Cramps. J Gen Intern Med 13: 600-606

McHugh JC, Reilly RB, Connolly S (2011): Examining the effects of age, sex, and body mass index on normative median motor nerve excitability measurements. Clin Neurophysiol 122: 2081-2088

Menon P, Kiernan MC, Vucic S (2014): Cortical dysfunction underlies the development of the split-hand in amyotrophic lateral sclerosis. PloS One $\underline{9}$ (1): e87124

Miller TM, Layzer RB (2005): Muscle cramps. Muscle Nerve 32: 431-442

Minetto MA, Holobar A, Botter A, Ravenni R, Farina D (2011): Mechanisms of cramp contractions: peripheral or central generation? J Physiol 589: 5759-5773

Mogyoros I, Kiernan MC, Burke D (1996): Strength-duration properties of human peripheral nerve. Brain 119: 439-447 
Mogyoros I, Kiernan MC, Burke D, Bostock H (1998): Strength-duration properties of sensory and motor axons in amyotrophic lateral sclerosis. Brain 121: 851-859

Moosmang S, Stieber J, Zong X, Biel M, Hofmann F, Ludwig A (2001): Cellular expression and functional characterization of four hyperpolarization-activated pacemaker channels in cardiac and neuronal tissues. Eur J Biochem FEBS 268: 1646-1652

Munsch T, Pape HC (1999): Modulation of the hyperpolarization-activated cation current of rat thalamic relay neurones by intracellular pH. J Physiol $\underline{519}$ Pt 2: 493-504

Naylor JR, Young JB (1994): A general population survey of rest cramps. Age Ageing $\underline{23}$ : $418-420$

Okuda B, Kodama N, Tachibana H, Sugita M (1997): Motor neuron disease following generalized fasciculations and cramps. J Neurol Sci 150: 129-131

Pape HC (1996): Queer current and pacemaker: the hyperpolarization-activated cation current in neurons. Annu Rev Physiol 58: 299-327

Park YA, Hay SN, King KE, Matevosyan K, Poisson J, Powers A, Sarode R, Shaz B, Brecher ME (2009): Is it quinine TTP/HUS or quinine TMA? ADAMTS13 levels and implications for therapy. J Clin Apheresis 24: 115-119

Raymond SA (1979): Effects of nerve impulses on threshold of frog sciatic nerve fibres. J Physiol 290: 273-303

Scicchitano P, Carbonara S, Ricci G, Mandurino C, Locorotondo M, Bulzis G, Gesualdo M, Zito A, Carbonara R, Dentamaro I et al. (2012): HCN channels and heart rate. Mol Basel Switz 17: 4225-4235

Serrao M, Rossi P, Cardinali P, Valente G, Parisi L, Pierelli F (2000): Gabapentin treatment for muscle cramps: an open-label trial. Clin Neuropharmacol 23: 45-49

Singh V, Gibson J, McLean B, Boggild M, Silver N, White R (2011): Fasciculations and cramps: how benign? Report of four cases progressing to ALS. J Neurol 258: 573-578 
Stevens DR, Seifert R, Bufe B, Müller F, Kremmer E, Gauss R, Meyerhof W, Kaupp UB, Lindemann B (2001): Hyperpolarization-activated channels HCN1 and HCN4 mediate responses to sour stimuli. Nature $\underline{413}$ : 631-635

Stieber J, Herrmann S, Feil S, Löster J, Feil R, Biel M, Hofmann F, Ludwig A (2003): The hyperpolarization-activated channel HCN4 is required for the generation of pacemaker action potentials in the embryonic heart. Proc Natl Acad Sci 100: 15235-15240

Tahmoush AJ, Alonso RJ, Tahmoush GP, Heiman-Patterson TD (1991): Crampfasciculation syndrome: a treatable hyperexcitable peripheral nerve disorder. Neurology 41: 1021-1024

Taylor JL, Burke D, Heywood J (1992): Physiological evidence for a slow K+ conductance in human cutaneous afferents. J Physiol 453: 575-589

Tomlinson S, Burke D, Hanna M, Koltzenburg M, Bostock H (2010a): In vivo assessment of HCN channel current $(\mathrm{I}(\mathrm{h}))$ in human motor axons. Muscle Nerve 41: 247-256

Tomlinson SE, Tan SV, Kullmann DM, Griggs RC, Burke D, Hanna MG, Bostock H (2010b): Nerve excitability studies characterize Kv1.1 fast potassium channel dysfunction in patients with episodic ataxia type 1. Brain J Neurol 133: 3530-3540

Trevillion L, Howells J, Burke D (2007): Outwardly rectifying deflections in threshold electrotonus due to K+ conductances. J Physiol 580: 685-696

Trevillion L, Howells J, Bostock H, Burke D (2010): Properties of low-threshold motor axons in the human median nerve. J Physiol $\underline{588}$ : 2503-2515

Vagg R, Mogyoros I, Kiernan MC, Burke D (1998): Activity-dependent hyperpolarization of human motor axons produced by natural activity. J Physiol 507: 919-925

Verdru P, Leenders J, Van Hees J (1992): Cramp-fasciculation syndrome. Neurology 42: 1846-1847

Vogel P: Kursbuch Klinische Neurophysiologie: EMG - ENG - Evozierte Potentiale. 3. Auflage; Georg Thieme Verlag, Stuttgart 2011 
Vucic S, Cheah BC, Yiannikas C, Vincent A, Kiernan MC (2010): Corticomotoneuronal function and hyperexcitability in acquired neuromyotonia. Brain J Neurol 133: 2727-2733

Wahl-Schott C, Biel M (2009): HCN channels: structure, cellular regulation and physiological function. Cell Mol Life Sci CMLS $\underline{66}$ : 470-494

Yang Q, Kaji R, Hirota N, Kojima Y, Takagi T, Kohara N, Kimura J, Shibasaki H, Bostock H (2000): Effect of maturation on nerve excitability in an experimental model of threshold electrotonus. Muscle Nerve 23: 498-506

Yang Q, Kaji R, Takagi T, Kohara N, Murase N, Yamada Y, Seino Y, Bostock H (2001): Abnormal axonal inward rectifier in streptozocin-induced experimental diabetic neuropathy. Brain J Neurol 124: 1149-1155

Yao H, Donnelly DF, Ma C, LaMotte RH (2003): Upregulation of the hyperpolarizationactivated cation current after chronic compression of the dorsal root ganglion. J Neurosci 23: 2069-2074

Yu X, Duan K-L, Shang C-F, Yu H-G, Zhou Z (2004): Calcium influx through hyperpolarization-activated cation channels (I(h) channels) contributes to activity-evoked neuronal secretion. Proc Natl Acad Sci 101: 1051-1056

Zierz S: Muskelerkrankungen. 3. Auflage; Georg Thieme Verlag, Stuttgart 2003

Zong X, Stieber J, Ludwig A, Hofmann F, Biel M (2001): A single histidine residue determines the $\mathrm{pH}$ sensitivity of the pacemaker channel HCN2. J Biol Chem 276: 63136319 


\section{Danksagung}

An dieser Stelle möchte ich Herrn Prof. Dr. med. Walter Paulus für die Möglichkeit danken, dass ich in seiner Abteilung diese Dissertation anfertigen konnte.

Mein besonderer Dank gilt Herrn Dr. med. Dirk Czesnik für die sehr gute Betreuung und für die Zeit, die er sich stets für mich und meine Fragen genommen hat.

Ich bedanke mich außerdem beim gesamten Team der Abteilung Klinische Neurophysiologie und insbesondere bei Julia Graf für die gute Zusammenarbeit und die Hilfe bei der Patientenorganisation. 


\section{Lebenslauf}

Ich wurde am 7. November 1990 in Kassel geboren. Von September 1997 bis Juli 2001 besuchte ich die Grundschule Regenbogenschule in Lohfelden und wechselte im August 2001 auf das Engelsburg-Gymnasium in Kassel, wo ich 2009 meine allgemeine Hochschulreife erwarb. Zum Wintersemester 2009/10 begann ich das Humanmedizinstudium an der Georg-August-Universität in Göttingen. Im Januar 2010 wurde ich als Stipendiatin in die Studienstiftung des deutschen Volkes aufgenommen. Den Ersten Abschnitt der Ärztlichen Prüfung schloss ich nach dem Sommersemester 2011 mit der Note 1,5 ab. Von April 2012 bis Februar 2014 habe ich als Doktorandin in der Klinik für Klinische Neurophysiologie der Universitätsmedizin Göttingen an einer klinischen Studie zum benignen Faszikulations-Crampus-Syndrom gearbeitet. Im November 2015 habe ich mein Studium an der Georg-August-Universität Göttingen abgeschlossen und arbeite seit Januar 2016 als Assistenzärztin in der Inneren Medizin im St. Bernward Krankenhaus Hildesheim. 